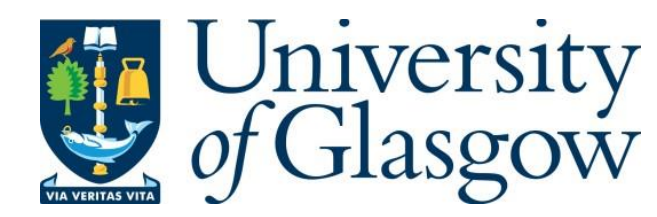

Raza, M., Aslam, N., Le-Minh, H., Hussain, S., Cao, Y. and Khan, N. M. (2017) A critical analysis of research potential, challenges and future directives in industrial wireless sensor networks. IEEE Communications Surveys and Tutorials, (doi:10.1109/COMST.2017.2759725).

There may be differences between this version and the published version. You are advised to consult the publisher's version if you wish to cite from it.

http://eprints.gla.ac.uk/149414/

Deposited on: 9 October 2017

Enlighten - Research publications by members of the University of Glasgow http://eprints.gla.ac.uk 


\title{
A Critical Analysis of Research Potential, Challenges and Future Directives in Industrial Wireless Sensor Networks
}

\author{
Mohsin Raza, Student Member, IEEE, Nauman Aslam, Member, IEEE, Hoa Le-Minh, Member, IEEE, Sajjad \\ Hussain, Member, IEEE, Yue Cao, Member, IEEE, and Noor Muhammad Khan, Senior Member, IEEE,
}

\begin{abstract}
In recent years, Industrial Wireless Sensor Networks (IWSNs) have emerged as an important research theme with applications spanning a wide range of industries including automation, monitoring, process control, feedback systems and automotive. Wide scope of IWSNs applications ranging from small production units, large oil and gas industries to nuclear fission control, enables a fast-paced research in this field. Though IWSNs offer advantages of low cost, flexibility, scalability, selfhealing, easy deployment and reformation, yet they pose certain limitations on available potential and introduce challenges on multiple fronts due to their susceptibility to highly complex and uncertain industrial environments. In this paper a detailed discussion on design objectives, challenges and solutions, for IWSNs, are presented. A careful evaluation of industrial systems, deadlines and possible hazards in industrial atmosphere are discussed. The paper also presents a thorough review of the existing standards and industrial protocols and gives a critical evaluation of potential of these standards and protocols along with a detailed discussion on available hardware platforms, specific industrial energy harvesting techniques and their capabilities. The paper lists main service providers for IWSNs solutions and gives insight of future trends and research gaps in the field of IWSNs.
\end{abstract}

Index Terms-Automation, IEEE802.15.4e, energy harvesting, IWSNs, ISA100.11a, MAC, TDMA, WirelessHART, WSNs, Zigbee, 6LoWPAN, CSMA/CA, Castalia, Fieldbus, Ethernet, Mesh, Tree, Star, Bus, Flat Architecture, Hierarchical,security, congestion, Wi-Fi, Bluetooth, UWB, Wasp Mote, Re-mote, OpenMote, SunSPOT, OMNeT++, MF, MiXiM, OPNET, Gradient, Flat, Proactive routing, Data centric, PV,VLC, IoT, Cognitive sensor networks, 6lo WG, OpenWSN.

\section{INTRODUCTION}

$\mathbf{I}$ NDUSTRIES have always been under continuous improvements since the very beginning of the industrial era. This gradual improvement is undoubtedly the outcome of continuous technology development in this field, which has kept the industries on its toes, looking for new methods for improvement of productivity and operational efficiency. More recently the continuous quality improvement has become the

Mohsin Raza and Hoa Le-Minh are with Department of Math, Physics and Electrical Engineering, Northumbria University, Newcastle, UK, e-mail: (mohsinraza119@gmail.com, hoa.le-minh@northumbria.ac.uk)

Nauman Aslam and Yue Cao are with Department of Computer and Information Sciences, Northumbria University, Newcastle, UK, e-mail: (nauman.aslam,yue.cao)@northumbria.ac.uk.

Sajjad Hussain is with the Department of Electronic and Nanoscale Engineering, University of Glasgow, Glasgow, UK, e-mail: Sajjad.Hussain@glasgow.ac.uk.

Noor M. Khan is with the Department of Electrical Engineering, Capital University of Science and Technology, Islamabad, Pakistan, e-mail: noor@IEEE.org. only mean to survive in the industrial race [1]. The past few years have resulted in vast expansion in industries. This expansion equipped the industries with the latest technology at hand, to develop self-sufficient, spontaneous and computerized work environments. Moreover, with the successful incorporation of advance automation and process control, the productivity and products' quality has greatly improved [2]. These improvements, though highly impressive yet add high complexity to the industrial processes and in some cases even challenge the sufficiency of existing technologies to cope with these rapid changes.

With the evolution of industries, new dimensions of research have surfaced. In recent years, the IWSNs have emerged as an efficient and cost effective solution for industrial automation and process control [3], [4], [5], [6]. The advantages offered by IWSNs have appeared to be a reason good enough to persuade many industries to its adoption, especially in low data rate applications [7], [8], [9]. One of the major factors contributing to the popularity of IWSNs is its low installation cost [10]. Compared to the cabling and maintenance costs of wired networks (up to $€ 4337$ per meter [11]), the wireless networking technologies offer a very small cost in fraction of a euro for per meter of wireless connectivity. Apart from the cost, the scalable nature of IWSNs make it an ideal candidate for present as well as future dynamic industrial environments [12], [13], [14], [15]. Furthermore, IWSNs offer many advantages, including flexibility, self-organization, low cost of installation, localized processing, interoperability and easy deployment. Despite these significant benefits of the technology, it suffers from constrained communication range, small memory, delay, limited bandwidth, reliability issues, limited battery capacity, security threats and interconnectivity issues [16]. Among all the afore-mentioned factors, while some favor the adoption of IWSNs in many applications, others open new research challenges to be dealt with [17], [18], [19].

Past few years have been very productive in addressing many challenges presented by IWSNs. The main developments witnessed until 2012 were carefully transformed to the IEEE Wireless Personal Area Network (WPAN) standard 802.15.4e [20], primarily targeting the industrial applications. Most of the amendments listed in this standard further improve the long chain of existing WPAN standards, [21], [22], [23], for industrial applications. Many industrial solutions based on these standards also emerged. Some significant contributions in- 


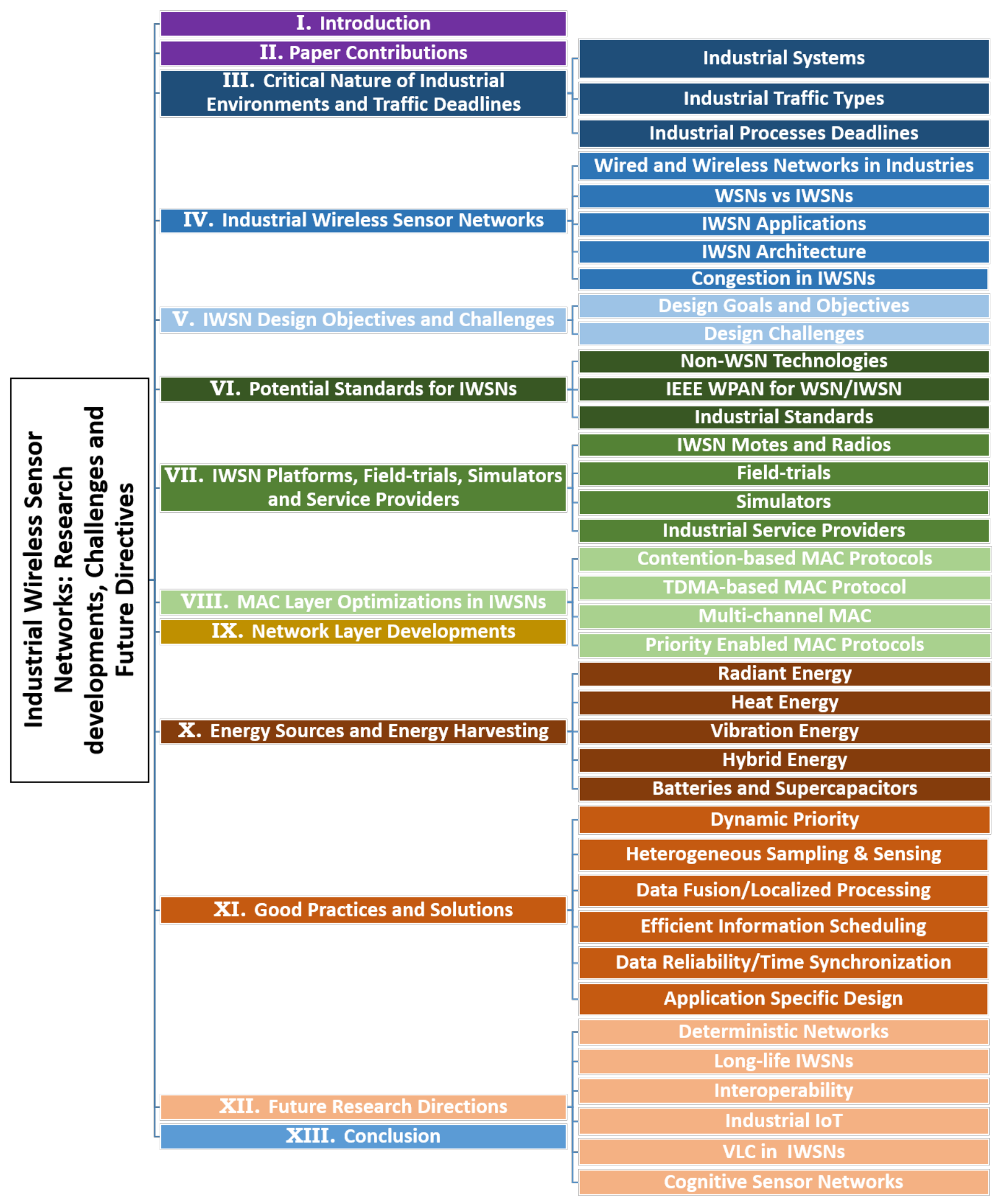

Fig. 1: Flow of the paper 
cluded, Zigbee, WirelessHART, ISA100.11a, 6LoWPAN WiaPA and Optimization of Communication for Ad hoc Reliable Industrial network (OCARI) [24], [25], [26], [27], [28], [29]. Moreover, research and development, during past three years, has significant impact in improving the IWSNs credibility for process control and automation. A keen and persistent trend in research developments was witnessed in these years, resulting in significant improvements in MAC protocols, network layer optimizations, energy harvesting techniques and incorporation of new technologies in industrial wireless networks [30], [31], [32].

These significant research developments in IWSNs have given new heights to this market, resulting in a momentous rise in its projected value ranging from $\$ 944.92$ million to $\$ 3.795$ Billion in coming years [33], [34]. However, it is also expected that the projection would highly depend on the research trends and significance of improvements one witnesses in upcoming years. To cope with the projected market trends, satisfy demands of more sophisticated industrial applications and to meet the crucial deadlines in highly sensitive industrial atmosphere, a dedicated research targeting reliability, real-time data delivery, incorporation of modular design and interoperability in IWSNs is much needed.

The rest of the paper is organized as follows: Section II discusses the main contributions of the paper. Section III categorizes industrial Systems and traffic generated in these system according to priority requirements. It also lists deadlines for selected industrial processes and discusses the failure consequences. Section IV gives an overview of IWSNs. Section $\mathrm{V}$ covers the design objectives and main challenges in IWSNs. Section VI discusses the existing work, standards and industrial protocols for IWSNs. Section VII gives overview of available industrial motes and their technical specifications. It also lists the IWSNs based solution providers for automation and process industry. Section VIII discusses MAC layer optimizations and research developments over the years. Network Layer developments are discussed in Section IX. Energy harvesting techniques for IWSNs are discussed in section X. Good practices and design solutions in IWSNs are discussed in section XI. Section XII gives an insight of future research directions in IWSNs. Finally, section XIII concludes the discussion and gives final remarks.

To give better understanding of flow of the paper, the taxonomy of the paper is represented graphically in Fig. 1.

\section{Key COntRibutions}

This paper provides a detailed description of IWSNs and its relevant areas of research and offers a wider perspective on advancements in these domains. To justify the contribution of the paper, some recently published key research articles, surveys and studies are thoroughly evaluated and main contribution of the published papers are highlighted in comparison to this paper. For the evaluation purposes main strengths and weaknesses of the published research are thoroughly discussed. Apart from this, in reference to earlier surveys published, Table I is formulated to present the contribution of this paper in comparison to other review papers. Out of the nineteen selected surveys, nine are published in IEEE communication surveys and Tutorials in the years from 2010 to 2016, five are published in IEEE transactions and IEEE Magazines, three in Elsevier journals and two in other journals. A brief description of contributions and shortcomings of these surveys and research articles are listed as follows.

In [16], authors make pioneer contribution in the field, and present an overview of IWSNs highlighting challenges and technical approaches in IWSNs. The paper discusses challenges in IWSNs in comparison to design goals. Paper also discusses the wireless standards and protocols and briefly touches energy harvesting. However, in this paper MAC layer developments are not discussed. Furthermore, most of the discussed topics cover a brief description and lack comprehensive details about the research.As the paper was published in 2009, there is abundance of improvements which address the needs of communication optimization, priority systems and appropriate traffic segmentation in industrial environments which are proposed afterwards and hence cannot be part of the discussion in this paper. The presented standards and industrial protocols have also seen a significant change in terms of channel access and hence new issues have developed which could not have been part of the discussion earlier.

In [19], authors present an industrial perspective of WSNs and discuss its applications in industrial environments. The paper discusses emerging challenges and expectations in IWSNs and lists expected operation of different layers. A brief discussion on the standards and protocols is also provided. However, the paper gives a direct approach to the possible problems and offers very limited information on classification of various protocols with notable footprint. Some of the key research areas in IWSNs including energy harvesting, WSN platforms, available radios and potential technologies are not discussed due to the scope of the paper.

In [25], authors discuss various WSN protocols and standards and give an insight on the suitability of these standards in industrial environments. The paper also provides a detailed discussion on the OCARI technology and lists its specifications, suitability and network topology. However, the paper did not mention any information on the research developments and proposed protocols over the years. The scope of the paper is limited and some prominent aspects like industrial requirements, IWSN platforms, energy harvesting, industrial deadlines, MAC developments etc. are not included.

In [35], authors present an introduction of WSNs and give a detailed discussion on the energy harvesting techniques in WSNs. The renewable energy resources are discussed in detail however, the discussion is limited in terms of broader perspective of IWSNs and no information on industrial requirements, MAC developments, challenges and design goals, standards and research developments is presented.

In [36], authors discuss in detail the various MAC layer developments in WSNs and discuss the IEEE standards and well known protocols in conventional and industrial WSNs. However, the paper does not specifically target IWSNs and provide a generalized discussion on WSNs. The paper also does not discuss the primary challenges in IWSNs, process control requirements, energy harvesting, IWSN platforms and 
TABLE I: Comparison of this Paper with other Surveys and review papers

\begin{tabular}{|c|c|c|c|c|c|c|c|c|c|c|c|c|c|c|c|c|c|c|c|c|}
\hline $\begin{array}{l}\text { Attributes/ } \\
\text { Main contributions }\end{array}$ & $\begin{array}{l}\text { This } \\
\text { Paper }\end{array}$ & [35] & [16] & [19] & [25] & [36] & [37] & [38] & [39] & [40] & [41] & [42] & [43] & [44] & {$[45]$} & [46] & [47] & [48] & [49] & [50] \\
\hline $\begin{array}{l}\text { Future Technologies for I- } \\
\text { WSNs }\end{array}$ & $\checkmark$ & $\mathrm{N}$ & $\mathrm{N}$ & $\mathrm{N}$ & $\mathrm{N}$ & $\mathrm{N}$ & $\mathrm{N}$ & $\mathrm{N}$ & $\mathrm{N}$ & $\mathrm{N}$ & $\mathrm{N}$ & $\mathrm{N}$ & $\mathrm{N}$ & $\mathrm{N}$ & $\mathrm{N}$ & $\mathrm{N}$ & $\mathrm{N}$ & $\mathrm{N}$ & $\mathrm{N}$ & $\mathrm{N}$ \\
\hline $\begin{array}{l}\text { Industrial systems, priori- } \\
\text { ties of affiliated traffic and } \\
\text { Time deadlines for various } \\
\text { industrial processes }\end{array}$ & $\checkmark$ & $\mathrm{N}$ & $\mathrm{N}$ & $\mathrm{N}$ & $\mathrm{N}$ & $\mathrm{N}$ & $\mathrm{N}$ & $\mathrm{N}$ & $\mathrm{N}$ & $\mathrm{N}$ & $\mathrm{N}$ & $\mathrm{N}$ & $\mathrm{N}$ & $\mathrm{N}$ & $\mathrm{N}$ & $\mathrm{N}$ & $\mathrm{N}$ & $\mathrm{N}$ & $\mathrm{N}$ & $\mathrm{N}$ \\
\hline $\begin{array}{l}\text { Priority criteria based di- } \\
\text { vision of wireless commu- } \\
\text { nication traffic }\end{array}$ & $\checkmark$ & $\mathrm{N}$ & $\mathrm{N}$ & $\mathrm{N}$ & $\mathrm{N}$ & $\mathrm{N}$ & $\mathrm{N}$ & $\mathrm{N}$ & $\mathrm{N}$ & $\mathrm{N}$ & $\mathrm{N}$ & $\mathrm{P}$ & $\mathrm{N}$ & $\mathrm{N}$ & $\mathrm{N}$ & $\mathrm{N}$ & $\mathrm{N}$ & $\mathrm{N}$ & $\mathrm{N}$ & $\mathrm{N}$ \\
\hline $\begin{array}{l}\text { Review of IWSNs tech- } \\
\text { nology and transition from } \\
\text { WSNs and wired network- } \\
\text { s }\end{array}$ & $\checkmark$ & $\mathrm{N}$ & $\mathrm{N}$ & $\mathrm{N}$ & $\mathrm{N}$ & $\mathrm{N}$ & $\mathrm{N}$ & $\mathrm{N}$ & $\mathrm{N}$ & $\mathrm{N}$ & $\mathrm{N}$ & $\mathrm{N}$ & $\checkmark$ & $\mathrm{N}$ & $\mathrm{N}$ & $\mathrm{N}$ & $\mathrm{N}$ & $\mathrm{N}$ & $\mathrm{N}$ & $\mathrm{N}$ \\
\hline $\begin{array}{l}\text { Taxonomy of MAC proto- } \\
\text { cols }\end{array}$ & $\checkmark$ & $\mathrm{N}$ & $\mathrm{N}$ & $\mathrm{N}$ & $\mathrm{N}$ & $\mathrm{N}$ & $\mathrm{N}$ & $\checkmark$ & $\mathrm{N}$ & $\mathrm{N}$ & $\checkmark$ & $\mathrm{N}$ & $\mathrm{N}$ & $\mathrm{N}$ & $\mathrm{N}$ & $\mathrm{N}$ & $\mathrm{N}$ & $\mathrm{N}$ & $\mathrm{N}$ & $\mathrm{N}$ \\
\hline $\begin{array}{l}\text { Classification of well- } \\
\text { known MAC protocols }\end{array}$ & $\checkmark$ & $\mathrm{N}$ & $\mathrm{N}$ & $\checkmark$ & $\mathrm{N}$ & $\checkmark$ & $\mathrm{N}$ & $\checkmark$ & $\mathrm{N}$ & $\mathrm{N}$ & $\checkmark$ & $\mathrm{N}$ & $\mathrm{N}$ & $\mathrm{P}$ & $\checkmark$ & $\mathrm{N}$ & $\mathrm{N}$ & $\mathrm{N}$ & $\mathrm{N}$ & $\mathrm{N}$ \\
\hline $\begin{array}{l}\text { Review of IEEE standard- } \\
\mathrm{s} \text { and Industrial protocols } \\
\text { for IWSNs }\end{array}$ & $\checkmark$ & $\mathrm{N}$ & O-D & $\checkmark$ & $\checkmark$ & $\checkmark$ & $\mathrm{P}$ & $\mathrm{N}$ & $\mathrm{N}$ & $\mathrm{N}$ & $\mathrm{N}$ & $\checkmark$ & $\mathrm{N}$ & $\mathrm{N}$ & $\mathrm{N}$ & $\mathrm{N}$ & $\mathrm{N}$ & $\mathrm{N}$ & $\mathrm{N}$ & $\checkmark$ \\
\hline $\begin{array}{l}\text { Review and classification } \\
\text { of Energy sources and En- } \\
\text { ergy harvesting in IWSNs }\end{array}$ & $\checkmark$ & $\checkmark$ & $\mathrm{P}$ & $\mathrm{N}$ & $\mathrm{N}$ & $\mathrm{N}$ & $\mathrm{N}$ & $\mathrm{N}$ & $\checkmark$ & $\mathrm{N}$ & $\mathrm{N}$ & $\mathrm{N}$ & $\mathrm{N}$ & $\mathrm{N}$ & $\mathrm{N}$ & $\checkmark$ & $\checkmark$ & $\checkmark$ & $\mathrm{P}$ & $\mathrm{N}$ \\
\hline & \multicolumn{5}{|c|}{$\checkmark:$ Covered } & \multicolumn{5}{|c|}{ N: Not Covered } & \multicolumn{5}{|c|}{ P: Partially Covered } & \multicolumn{5}{|c|}{ O-D: Out-Dated } \\
\hline
\end{tabular}

noteworthy technologies for future IWSNs.

In [37], authors present a survey of WSN protocols and standards and discuss the operation of the selected OSI layers. However, the discussion only focuses on Carrier Sense Multiple Access (CSMA) based communication which is not suitable for majority of industrial applications. Furthermore, the scope of the paper is limited in terms of providing a broader perspective of the research areas and limits the discussion to a specific domain in WSNs.

In [38], authors present MAC layer development for mission critical applications over the years. The paper does not take into consideration the industrial standards and other research areas in IWSNs including process control requirements, IWSN platforms, industrial standards, energy harvesting etc.

In [39], [46], [47] and [48], authors present a survey of energy harvesting sensor nodes. The papers discuss the potential of various energy harvesting techniques for wireless motes and provide application areas in WSNs. However, as the scope of the papers suggest, the discussion is limited and does not provide an insight into the industrial requirements and energy harvesting potential of various techniques in accordance with the industrial applications. Furthermore, the papers offer little information regarding the broader perspective of IWNs and key research areas in IWSNs are not part of the papers under consideration.

In [40], authors present a survey on use of WSNs in industrial automation and process control. The paper presents an overview of different WSN communication technologies, and discusses the possible challenges and solutions. However, the discussion provided in the paper does not offer a consolidated information on IWSNs and fails to discuss the primary IEEE and industrial standards. The paper also does not provide any discussion on the role of MAC layer given its significance in IWSNs. Other key areas in IWSNs are also not discussed.

In [41], authors present a detailed discussion on the latency issues of asynchronous MAC protocols for delay sensitive networks. The paper presents a classification of MAC protocols and provides a comprehensive discussion on the latency issues 
in MAC. However, due to the scope of the paper the discussion lacks a detailed survey of technologies and research areas in IWSNs.

In [42], authors discuss suitability of WSNs for industrial automation. The paper discusses different wireless standards including Bluetooth and WPAN for communication in IWSNs. The paper also discusses some of the industrial standards in IWSNs and highlights their key characteristics. However, the scope is limited and the potential of WSN technology and its suitability in industrial applications is not discussed in detail. Furthermore, some of the key research areas including energy harvesting, IWSN platforms, MAC developments and the Time Division Multiple Access (TDMA) based communication in IWSNs is beyond the scope of this paper.

In [43], authors discuss the suitability of wired and wireless sensor networks in industrial applications. The paper briefly discusses ethernet and fieldbus protocols. The paper also discusses the potential of WSNs in industrial applications and possible challenges. However, the paper does not cover any industrial standards, MAC layer developments, and wireless communication technologies. Some other key research areas in IWSNs like IWSN motes and energy harvesting are not discussed as well.

In [44] and [45] authors present survey and developments in MAC protocols, however, in [44] the MAC protocols are not thoroughly analysed and paper fails to offer a suitable MAC layer protocol classifications. In [45] a detailed discussion on the MAC protocols can be found however, the paper does not offer substantial insight in the industrial requirements for MAC protocols and fails to relate the discussion in industrial perspective. Furthermore, both the papers lack a detailed discussion on the IWSNs and its various significant research aspects.

In [50] authors survey the suitability of WSN in factory automation. The paper discusses the possible challenges and reliability issues of WSNs in industrial environments. Paper also presents the security issues in using IWSNs. A brief discussion on selected industrial standards is also provided. However, paper lacks comprehensive background of IWSNs and does not cover any details on categorization of industrial applications, time deadlines, requirements, MAC layer developments, hardware specifications, mote details, energy harvesting and potential technologies for IWSNs.

In this paper, substantial effort has been made to, firstly present a through review of up-to-date IWSN technology; secondly provide critical analysis and summary of exiting works, and thirdly highlight potential research areas and articulate significant research challenges Main contributions of the paper are listed as under.

- The paper covers a detailed review of IWSNs, presents IWSN architecture, discusses its strengths and limitations, provides an insight on ongoing transition from WSNs to IWSNs and wired networks to wireless networks, presents significant wired and wireless protocols and standards and lists the key application areas of IWSNs for industrial environments.

- Provides a division of network traffic involved in IWSNs into logical levels. The traffic types (levels) are defined based on the critical nature, time deadlines and reliability requirements for each of the targeted application areas and industrial systems. Furthermore, practical examples from industrial processes are presented with the time deadlines, reliability, and security requirements

- A detailed review of key IEEE standards and industrial protocols and their suitability for industrial applications is provided. A taxonomy of significant developments, information of standards and industrial protocols, development of wireless motes, market trends, IWSN market value and expected futuristic technologies for IWSNs is presented along with the time information.

- A taxonomy of MAC protocols to categorize different MAC based developments is presented along with the classification of significant MAC protocols. The classification is based on the application area, channel access scheme, frequency channels, priority scheduling and latency information.

- A taxonomy of Routing protocols is presented to categorize different network layer developments. Each subcategory of routing protocols is thoroughly discussed and flagship routing protocols for each category are listed.

- The hardware architecture of wireless motes, technical specifications and radio details of selected motes are presented. Some significant IWSNs based solutions providing companies are also discussed.

- The main energy sources in industrial environments are discussed. Different energy harvesting techniques are reviewed and categorized. The life cycle and energy specifications of energy harvesting techniques, usable and reusable batteries and super capacitors are listed.

- Extensive research challenges, design objectives and design solutions for IWSNs are presented.

- Possible future research themes and technologies for industrial networks including Internet of Things (IoT), Visible Light Communication (VLC), Cognitive Radio Sensor Networks (CRSNs), deterministic networks and long life IWSNs are presented.

\section{CRitical NATURE OF INDUSTRIAL ENVIRONMENTS AND TRAFFIC DEADLINES}

\section{A. Industrial Systems}

IWSNs offer services to specific range of applications, which are significantly different from the traditional WSNs. Therefore, based on the specific application characteristics, Quality of Service (QoS), latency and security requirements, the industrial systems are classified [19]. According to the International Society of Automation (ISA), the industrial systems can be distributed into six classes [51], [52]. This classification is based on the nature of application, standard operating procedure, access schemes, reliability, and latency requirements. These systems are listed as under.

1) Safety/Emergency systems: Safety/emergency systems handle issues of greater significance and of critical nature. For such systems, action on the developed situations, are required in matter of milliseconds. Any added delay can contribute to 
unwanted complications. Fire alarms, leakage of poisonous gases and emission of radiations are some of the examples of emergency systems.

2) Close loop regulatory control systems: Close loop regulatory systems require a periodic feedback for smooth running of the processes. Such systems include both sensor and actuator elements where a continuous feedback from the sensors is needed to maintain the desired response of the actuation part. Usually the time bounds between sensing values and making the desired corrections using actuators, based on the sensed values, are very low. Some examples of close loop regulatory systems include autonomous cars or piloted drive, motion adaptation for conveyor belt movements and affiliated robotics etc.

3) Close loop supervisory systems: Close loop supervisory systems also provide a feedback control like the regulatory systems, except, these systems are asynchronous in nature and a feedback mechanism is established when certain thresholds are violated. Since, these systems are less critical in nature compared to the regulatory control systems, therefore, time and reliability bounds are more relaxed. Examples of close loop supervisory control include slow changing and less critical processes like temperature control of a furnace or boiler etc.

4) Open loop control systems: The open loop control systems implement human operated process control. These systems, instead of automated analysis, rely on human intervention, where the operator after analysing the sensed data, takes the necessary action.

5) Alerting systems: In industries, alerting systems usually provide feedback of the sequential processes where regular or prompt feedback is established as a surety mechanism. Such systems offer tracking mechanism with regular feedbacks for different stages of the processes. In some cases, event-based alerting is also established.

6) Information gathering systems: Information gathering systems are used to collect sensor reading regarding nonactionable processes. The data gathering is targeted to provide the pattern observations over long period of time, which can serve as a baseline for the future changes and implementing long term plans. These systems and information gathered in similar systems is typically non-critical in nature and therefore, the data accumulation phases can span days. The accumulated data by information gathering systems usually undergoes a computer based diagnosis to devise the improvement plans on the basis of data analysis.

\section{B. Traffic Types in Industrial Systems}

A number of researches [30], [53], [54], [55] have categorized traffic in industrial environments in a number of groups depending on the type and critical nature of the traffic. In this paper, the traffic in an industrial setup, in reference to presented industrial systems, is categorized in six groups. These categories are defined on the basis of critical nature of information, reliability, time constraints, medium access control and channel access pattern. These traffic types are listed in most critical to least critical order.
1) Safety/Emergency traffic: The safety or emergency traffic is the traffic of highest priority, if mishandled, may threaten a human life or incur damages to a plant. It is usually asynchronous in nature and rarely triggers due to anomalies and hazards such as risk of explosion or severe electrical surges etc. Due to the sensitive nature of this traffic category, high reliability is expected and fail-safe link is established with multiple contingencies [55]. This type of traffic has highest priority and usually prioritized over the rest of the traffic. The time and reliability constraints of such industrial network traffic require careful modelling along with the prioritized access to communication channel [51].

2) Regulatory control traffic: The traffic originated from the systems running close loop regulatory control contribute significantly in density of IWSNs network traffic. There are two primary reasons: the sampling rate of the sensors involved in regulatory control is much higher and the information generated by these systems is periodic. The regulatory control traffic has much higher significance compared to other traffic types [52], except emergency, due to the strict bounds of the close loop systems. Furthermore, such control systems try to minimize the dead-time between two consecutive communications to optimize the performance of the close loop systems. Any negligence or delay in regulatory control traffic at network layer can lead to the safety or emergency trigger, which enhances the importance of this traffic. Since the regulatory control traffic, poses synchronous information load, therefore, it occupies constant bandwidth. Failure in communication may lead to the instability of the process control, therefore high reliability of such traffic is ensured [51], [55]

3) Supervisory control traffic: The supervisory control traffic is quite similar to regulatory control traffic except, it is asynchronous in nature. In this case, localized processing is incorporated to identify if the specified thresholds are violated in any manner. Based on the initial conditions (if the sensed values are within specified thresholds or not), the priority to traffic is assigned [374]. The behaviour of supervisory control traffic can be related to emergency traffic, however, due to less critical nature of applications at hand supervisory control traffic, depending on the conditions, is either modelled as regulatory control traffic [51], [373] (for value beyond critical thresholds) or asynchronous alerting traffic [55] (for value within the critical thresholds). Since the importance of this type of traffic is dependent on the critical nature of information, therefore, the critical and non-critical categories are dealt separately. In critical case the information is regularly reported from sensory data to control centre and requires higher level of reliability whereas for the less critical case asynchronous communication is established with less stringent reliability conditions.

4) Open loop control traffic: It is termed as a low risk control traffic with relatively relaxed time and reliability bounds [51], [12]. Since the failure in one or more communications won't have a significant impact on the implemented process control due to slow changing nature of the target control systems, therefore reliability is not as important as in case of emergency and regulatory systems. IWSNs in such systems mainly report the information of less critical nature to the 
TABLE II: Traffic Categories and affiliated attributes in Industrial Wireless Sensor Networks [30], [51], [53], [54], [55]

\begin{tabular}{|c|c|c|c|c|c|c|c|}
\hline \multirow[t]{2}{*}{ Sr. } & \multirow[t]{2}{*}{ Traffic category } & \multirow[t]{2}{*}{ Case } & \multirow[t]{2}{*}{ Priority } & \multirow[t]{2}{*}{ Applications } & \multicolumn{2}{|c|}{ Tolerance } & \multirow[t]{2}{*}{ Medium Access Control } \\
\hline & & & & & Time constraint & Reliability & \\
\hline 1. & $\begin{array}{l}\text { Safety/Emergency } \\
\text { Traffic [51], [53], } \\
{[55]}\end{array}$ & - & Very high & $\begin{array}{l}\text { Emergency/Alarms } \\
\text { Asynchronous }\end{array}$ & Few milliseconds & $\begin{array}{l}\text { High reliability } \\
\text { requirements }\end{array}$ & $\begin{array}{l}\text { Pilot channels, Dedicated } \\
\text { frequency, prioritized slot- } \\
\text { ted access }\end{array}$ \\
\hline 2. & $\begin{array}{l}\text { Regulatory control } \\
\text { traffic [51], [55] }\end{array}$ & - & High & $\begin{array}{l}\text { Close loop process } \\
\text { control / critical } \\
\text { feedback Periodic }\end{array}$ & $\begin{array}{l}\text { Tens of millisecond- } \\
\mathrm{s}\end{array}$ & $\begin{array}{l}\text { High reliability } \\
\text { requirements }\end{array}$ & $\begin{array}{l}\text { Slotted access using TD- } \\
\text { MA or high priority C- } \\
\text { SMA/ CA based channel } \\
\text { access with enabled re- } \\
\text { transmissions }\end{array}$ \\
\hline \multirow[t]{2}{*}{3.} & \multirow[t]{2}{*}{$\begin{array}{l}\text { Supervisory control } \\
\text { Traffic [51], [55] }\end{array}$} & i. Critical & High & $\begin{array}{l}\text { Close loop process } \\
\text { control / critical } \\
\text { feedback Periodic }\end{array}$ & $\begin{array}{l}\text { Tens of millisecond- } \\
\mathrm{s}\end{array}$ & $\begin{array}{l}\text { High reliability } \\
\text { requirements }\end{array}$ & $\begin{array}{l}\text { Slotted access using TD- } \\
\text { MA or high priority C- } \\
\text { SMA/ CA based channel } \\
\text { access with enabled re- } \\
\text { transmissions }\end{array}$ \\
\hline & & ii. Non-critical & Low & $\begin{array}{l}\text { Asynchronous occa- } \\
\text { sional feedbacks }\end{array}$ & Seconds to hours & $\begin{array}{l}\text { Low reliability } \\
\text { with occasional } \\
\text { packet misses }\end{array}$ & $\begin{array}{l}\text { CSMA/ CA based channel } \\
\text { access }\end{array}$ \\
\hline 4. & $\begin{array}{l}\text { Open loop control } \\
\text { traffic [51] }\end{array}$ & - & Medium & Periodic & Seconds-minutes & $\begin{array}{l}\text { Medium reliabili- } \\
\text { ty requirements }\end{array}$ & $\begin{array}{l}\text { Slotted ac- } \\
\text { cess/(CSMA/CA) based } \\
\text { channel access with high } \\
\text { priority overwrite ability }\end{array}$ \\
\hline \multirow[t]{2}{*}{5.} & \multirow[t]{2}{*}{$\begin{array}{l}\text { Alerting traffic [51], } \\
\text { [55] }\end{array}$} & i. Critical & Medium & Periodic & Seconds-minutes & $\begin{array}{l}\text { Medium reliabili- } \\
\text { ty requirements }\end{array}$ & $\begin{array}{l}\text { Slotted ac- } \\
\text { cess/(CSMA/CA) based } \\
\text { channel access with high } \\
\text { priority overwrite ability }\end{array}$ \\
\hline & & ii. Non-critical & Low & $\begin{array}{l}\text { Asynchronous occa- } \\
\text { sional feedbacks }\end{array}$ & Seconds to hours & $\begin{array}{l}\text { Low reliability } \\
\text { with occasional } \\
\text { packet misses }\end{array}$ & $\begin{array}{l}\text { CSMA/ CA based channel } \\
\text { access }\end{array}$ \\
\hline 6. & $\begin{array}{l}\text { Monitoring traffic } \\
{[51],[53],[55]}\end{array}$ & - & - & $\begin{array}{l}\text { Monitoring } \\
\text { Application } \\
\text { static feedback }\end{array}$ & minutes to hours & $\begin{array}{l}\text { Low reliability } \\
\text { requirements }\end{array}$ & $\begin{array}{l}\text { Best effort service, CS- } \\
\text { MA/CA based channel ac- } \\
\text { cess }\end{array}$ \\
\hline
\end{tabular}

control unit where an operator analyses the output [55]. One example of such control is the frequency component of legacy hydroelectric generation units. As the frequency changes occur as a function of output load on the system so these changes are relatively slow. Based on the feedback from the sensory elements, sampled frequency values are presented to operator, who decides whether an increase or decrease in water valve opening is needed to regulate the frequency of generated electric power. Since it is a human dependent response system, it can have unaccounted delays which are only affordable because of the less critical nature of this traffic [55]. In such cases, very occasional actions are expected in response to the information received. Nevertheless, the accumulation of readings from the sensors follows a periodic behaviour.

5) Alerting traffic: Alerting traffic follows a relatively low duty cycle where the amount of information communicating over the IWSNs is very much limited [51]. The frequency of communication can only be increased if certain anomalies occur. In case the information becomes critical the reliability and priority of this traffic is increased otherwise a relatively lower reliability assurance is needed and occasional failures in packets communications dont cause major problems [52]. This category of industrial communication is more related to emergency traffic in behaviour however, its intrinsic properties are very much different from emergency traffic. In case of anomalies the priority level of this traffic can be considered on the similar levels of that of supervisory control traffic.

6) Monitoring traffic: Monitoring traffic is mostly categorized as single way traffic as it is used to monitor the status of the processes having relatively less significance in the control and automation [51], [55]. In most cases, the information collected in monitoring systems, assist in the formation of future suggestions for system upgrades and improvements. The occasional packet failures are common in such systems and hence demand lower reliability bounds [55].

Traffic types discussed in this subsection along with the priority requirements, time constraints, reliability and medium access schemes, are represented in Table II.

\section{Critical Industrial Deadlines and Failure Consequences}

The above discussion categorizes the types of the traffic in an industrial environment, to define the priority levels, time deadlines, class of control systems and relative medium access schemes. In critical cases, a delay in conveying sensed information can result in damage to the equipment, may lead to an explosion or threat to a human life. Therefore it is important to properly identify different traffic types originated 
TABLE III: Typical end-to-end delay and update requirements for industrial processes [17], [42], [57], [58]

\begin{tabular}{|c|c|c|c|}
\hline Sensor Network Applications & Security Requirements [59], [60] & Update Frequency & Battery Lifetime [17] \\
\hline \multicolumn{4}{|l|}{ Monitoring and Supervision } \\
\hline Vibration sensor [17], [53] & Low & sec - days & up to 3 years \\
\hline Pressure sensor [17], [53] & Low & $1 \mathrm{sec}$ & up to 3 years \\
\hline Temperature sensor [17], [53] & Low & $5 \mathrm{sec}$ & up to 3 years \\
\hline Gas detection sensor [17], [53] & Low & $1 \mathrm{sec}$ & up to 3 years \\
\hline Others/Data acquisition & Low & $>100 \mathrm{~ms}$ & up to 3 years \\
\hline Maintenance diagnosis & Low & Sec-days & - \\
\hline \multicolumn{4}{|l|}{ Close Loop Control } \\
\hline Control valve [42], [53] & medium to high & $10-500 \mathrm{~ms}$ & 5 years \\
\hline Pressure sensor [17], [42], [53] & medium to high & $10-500 \mathrm{~ms}$ & 5 years \\
\hline Temperature sensor [17], [42], [53] & medium to high & $10-500 \mathrm{~ms}$ & 5 years \\
\hline Flow sensor [17], [42], [53] & medium to high & $10-500 \mathrm{~ms}$ & 5 years \\
\hline Torque sensor [17], [42], [53] & medium to high & $10-500 \mathrm{~ms}$ & 5 years \\
\hline Variable speed drive [53] & medium to high & $10-500 \mathrm{~ms}$ & 5 years \\
\hline Motion Control [42] & Isochronous & $250 \mu \mathrm{s}$ to $1 \mathrm{~ms}$ & up to 1 year \\
\hline Control Machine Tools [42] & High & $1 \mathrm{~ms}$ to $10 \mathrm{~ms}$ & up to 3 year \\
\hline \multicolumn{4}{|l|}{ Interlocking and Control } \\
\hline Proximity sensor [17], [42], [53] & medium to high & $10-250 \mathrm{~ms}$ & 5 years \\
\hline Motor [17], [42], [53] & medium to high & $10-250 \mathrm{~ms}$ & 5 years \\
\hline Valve [17], [42], [53] & medium to high & $10-250 \mathrm{~ms}$ & 5 years \\
\hline Protection relays [17], [42], [53] & medium to high & $10-250 \mathrm{~ms}$ & 5 years \\
\hline Machinery and tools & medium to high & $10 \mathrm{~ms}$ & up to 3 years \\
\hline Motion Control & medium to high & $1 \mathrm{~ms}$ & up to 3 years \\
\hline \multicolumn{4}{|l|}{ CAN bus Deadlines } \\
\hline Periodic Messages [58] & Medium & $5-20 \mathrm{~ms}$ & - \\
\hline Non-periodic Messages [58] & Medium & $5 \mathrm{~ms}$ & - \\
\hline
\end{tabular}

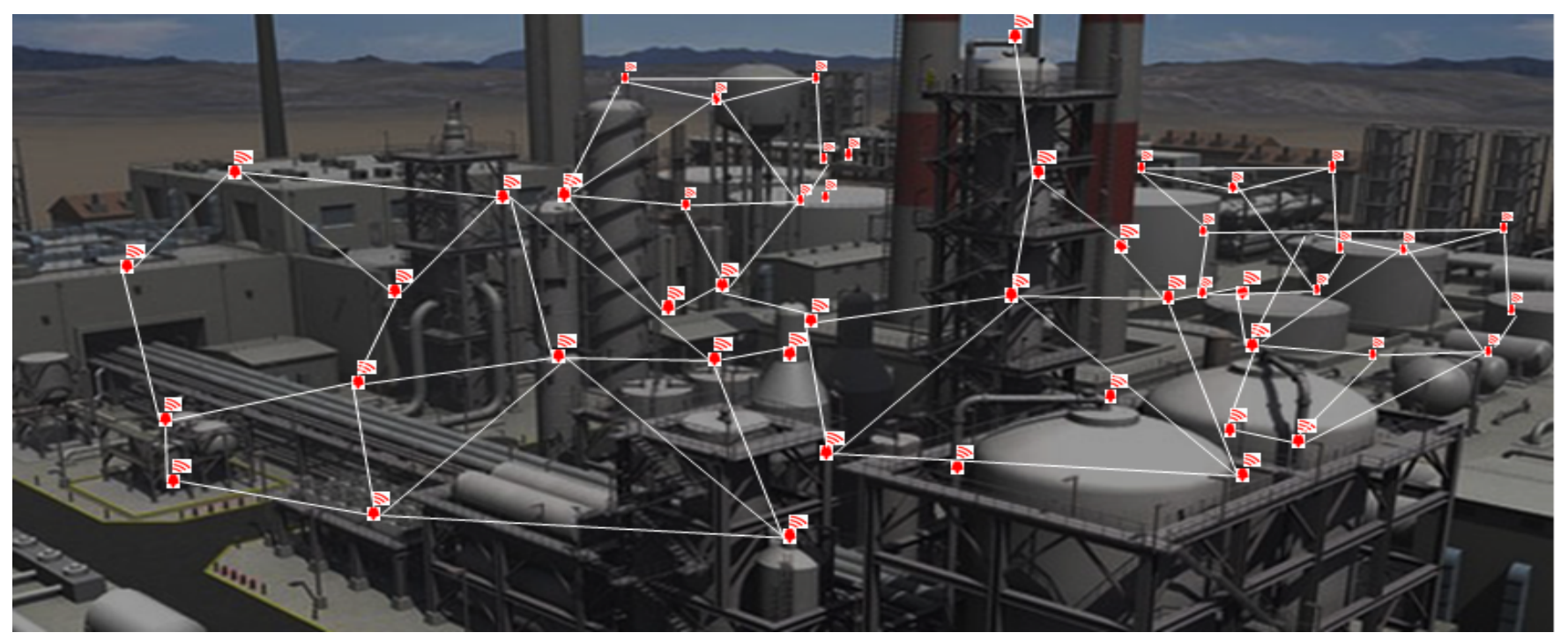

Fig. 3: A Typical Industrial Wireless Sensor Networks Setup [70] 


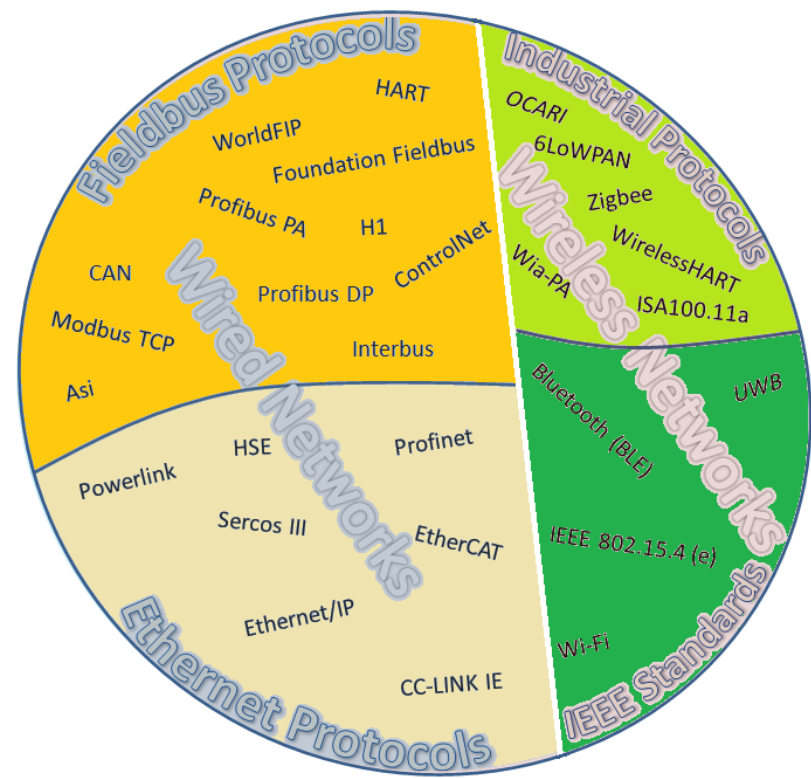

Fig. 2: Industrial Wired and Wireless Network technologies and Standards [16], [23], [26], [27], [28], [29], [37], [79],

[80], [81], [82], [83], [84], [85], [86], [87], [88], [89]

in a system to affiliate right level of importance to each traffic type. Although, the specification of needs and categorization of information in different types is step towards the right direction yet there is a lot of work needed in modeling the algorithms and establishing priority as well as reliability constraints acceptable to the control and automation community [56]. Moreover, the full potential of IWSNs must be exploited to offer a better solution than the existing, to meet the deadlines set forth for industrial process control, and automation.

Some of such requirements for different industrial equipment are listed in Table III. As Shown in Table III, even a particular sensing application has a broad range of parameters attached to it. For instance, considering the temperature sensing in close loop control, a wider range of update frequency is affiliated as represented in Table III. The reason is much more dependent on the core process and application area for which the temperature sensing is considered. To justify the variations, consider two application scenarios where in one case the temperature sensing is used in fractional distillation of crude oil and the other involves the operational temperature of pressurized flammable gases. The former is much more variation tolerant than the later as for fractional distillation some significant temperature variations can increase the level of impurities in different distilled oil products, which is undesirable but not hazardous. However, in case of dealing with pressurized flammable gases, the temperature variations are much more sensitive and can even cause fire. Therefore, in dealing with flammable gases, more frequent feedback is desirable. This example also signifies the need for priority of one information type over the other, as a mandatory part of wireless communication link for timely actions on critical processes. The presented information in Table III, gives an overview of possible deadlines in industrial environments, however, an application specific evaluation of different sensors is needed to better classify the significance of a particular sensing process, consequences of failure in its communication and system type to which this sensing operation belongs.

Apart from the time deadlines and frequency of communication of individual nodes, the battery-operated nature of IWSNs also affiliates a suitable value to lifetime of these nodes. Longer battery life ensures an uninterrupted operation of the processes and reduction in the maintenance cost. Recommendations for the desired battery lifetime of the sensor nodes in different industrial applications are presented in Table III, whereas a detailed discussion on the suitability of various available batteries and energy harvesting techniques is presented in Section X.

The importance of information communicated for the regulation of an industrial process can vary significantly within an industrial plant. Depending on the type of application at hand, and nature of its severity, it must be shielded from the intruders and cyber threats. Since, the external intervention can raise significant issues in the control of processes; a suitable protection from external threats must be implemented.

Unlike the wired networks where the intruders need to be physically connected to the networks, the wireless networks are much more susceptible to security threats, thus requiring more suitable security techniques [356]. The main security criteria for evaluation include confidentiality, integrity, authenticity and availability [57], [357]. The importance of these factors can be changed based on the application at hand. In the industrial environments information security has been considered an integral part of the standardization and is given higher preference for more critical applications [82]. With the rise in possible security threats, IWSNs appears to be more vulnerable to information tampering, node control, denial of service, flooding, radio interference, eavesdropping and traffic analysis [357], [37], [42] and hence require appropriate security and data encryption. A recommendation on security requirements for different applications is presented in Table III, whereas a detailed description of security features implemented in IWSNs are listed in Section IV-D(5).

\section{Industrial Wireless Sensor Networks}

\section{A. Wired and Wireless Networks in Industrial Environments}

The communication networks established in industrial environments can be broadly divided in two categories, wired and wireless. However, the strong interference experienced in industrial environment along with high performance demands make industrial solutions very challenging. It is for the same reason; wired solutions were preferred over wireless in the last decade. Many wired solutions were proposed to offer high-speed communication, deterministic reliability, and real time delivery [43]. The wired communication networks in the industries were designed to target four specific objectives [57] which included real-time assurance, guaranty for functional safety, guaranty for security and centralized supervisory control of decentralized processes. Further requirements included the ability of remote commissioning and maintenance of distributed automation systems. Over the years many wired standards and technologies were introduced to meet the stringent real-time and reliability requirements of the industrial 
processes. These wired network developments can broadly be classified in the fieldbus systems and Ethernet systems.

1) Fieldbus: Fieldbus systems have played a significant part in the industrial automation for a long time resulting in standardization of numerous technologies such as [73], [74]. Due a number of desirable characteristics such as deterministic behaviour, lesser sensitivity to electrical noise, simplified connectivity and ability to operate over long distance, fieldbus networks are widely used in the industrial environment to connect field level equipment including motors, transmitters, control valves, proximity sensors, accelerometers, encoders, monitoring and control devices. Some of the significant developments in the fieldbus systems offering complete protocol suit include PROFIBUS, CAN, P-Net, Interbus, SwiftNet, Fieldbus $\mathrm{H} 1$, and WorldFIP.

2) Ethernet: High data rates and larger bandwidth offered by Ethernet qualifies it to serve as a backbone of the industrial networks. Industrial Ethernet received wide acceptance for communication among Programmable Logic Controllers (PLC) and Supervisory Control And Data Acquisitions (SCADA) with TCP/IP enabled interlinking [43]. Furthermore, the switch-based architecture, remote diagnosis, and selfconfiguring tools of Ethernet offer significant improvement over the fieldbus networks. Therefore, the use of Ethernet at field level appeared to be a promising solution to the interconnectivity problem [75] between high-level (Ethernet) and low-level (Fieldbus) networks [76]. However, an accurate priori delay estimation for effective operation of supervisory control is imperative, therefore, the uncertainty in industrial Ethernet must be addressed. Later, to improve the suitability of Ethernet on field level, Real Time Ethernet (RTE) was introduced [77] which used TDMA based channel access scheme for improved reliability and predictable delays. Use of TDMA resulted in the synchronization issues, which were later addressed in IEEE1588 standard [78]. Over the years some significant Ethernet based developments include High Speed Ethernet (HSE), CC-LINK IE, EtherCAT and Sercos III.

Although, the wired networks offered modest data rates and reliability but failed in offering scalability, cost efficiency and efficient network deployment. All these factors forced the researchers to look into wireless communication solution for industrial automation. Cost efficiency, flexibility and selfhealing abilities of IWSNs among many others vouched its suitability for wider industrial application domains.

Suitability of IWSNs resulted in formation of many research groups leading to various industrial and IEEE standards. Some of the industrial protocols and standards include IEEE 802.15.4 and IEEE 802.15.4e, Zigbee, WirelessHART and ISA100.11a. A more detailed discussion can be found in Section VI, whereas a graphical representation of the existing industrial wired and wireless network communication standards and protocols are shown in Fig. 2.

\section{B. WSNs and IWSNS}

WSNs are a type of networks with spatially distributed autonomous devices working collaboratively to offer a variety of monitoring applications [58]. These autonomous devices, referred as nodes, serve as a the most appropriate technology to monitor large physical environments [61]. Each node in WSNs has a small range in tens of meters and information from source to destination (Gateway) is transmitted in multihop fashion [62]. This collaborative nature of WSNs allows the flexibility of adding new nodes to the network and operate in different network topologies [63], [64].

The transformation of WSNs from non-critical monitoring [65] applications to highly critical process control, automation and real time decision making [66], [67], [68], [69], pushed this technology to limits. Highly sensitive nature of industrial processes and plants add many constraints which are still a challenge for WSNs. Moreover, since most of the industrial environments have transformed into highly dynamic and vibrant processes, typical WSNs are no longer suitable to serve as a solution.

IWSNs are a special domain of WSNs which particularly targets industrial applications [71], [72]. A typical representation of IWSNs is presented in Fig. 3. The working principle of IWSNs is quite similar to that of WSNs. However, the need for strict timing deadlines, reliability constraints and critical nature of industrial applications makes IWSNs an entirely different research domain. As the industrial applications may involve close loop control systems and critical processes automation, the primary research focuses in IWSNs are reliability, realtime data delivery and deterministic network designs. In some cases, IWSNs also need a long network lifetime to synchronize the maintenance of wireless networks with the industrial equipment. Though the lifetime requirements highly depend on industrial applications, yet the technological improvements in industrial sector has significantly increased the maintenance and life cycles of industrial equipment which is no less than a challenge for IWSNs to meet with.

\section{IWSNs Applications}

At present, IWSNs are used in wide variety of applications. Some of the applications include, monitoring, open-loop and close-loop control of processes, and emergency response systems. A few of these scenarios are listed as under.

1) Area monitoring: One of the most common applications of the IWSNs is area monitoring. Such monitoring in industrial areas may involve sampling of some basic parameters of interest, like temperature, pressure etc. The primary objective of the sensors in similar scenario is to accumulate data over a period of time and relay the data to the destination using the established network. Such monitoring networks offer information over extended periods of time which can be used for the computer based analysis and future improvement and recommendations [61]. Similar networks can be used in the industry for slow changing processes where no direct action is required.

2) Air/water quality and waste monitoring: The pollutants from the industries cause environmental degradation and severe problems for living systems. To keep check of the pollutants in air IWSNs play a significant role. To evaluate air pollution levels and proportion of dangerous gases in 
the atmosphere, large scale wireless networks are formulated. These extended networks with a variety of sensors offer improved accuracy.

Like air, it is of utmost importance that the water quality is regularly checked so that the accurate records of the impurities, added in water, are maintained. For such purposes, wireless nodes are used to accumulate the data over time to provide the updated information on the water condition in the nearby lakes, rivers etc [165], [166]. Moreover, with the sensor nodes deployed and permanent monitoring stations established, accurate data can be collected. Deployment of the sensor nodes also cuts the burden of manual readings. Water wastes also contribute greatly to the surface as well as underground water which if accurately evaluated can present a number of ways to implement remedies.

3) Machine health monitoring: IWSNs have also been used to evaluate the machinery condition. Sensor nodes are deployed to evaluate conditions of various potentially important machinery parts and to evaluate the need for machinery maintenance [322]. The use of IWSNs also allow the feedback of the rotatory parts in machines which were once inaccessible using wired networks.

4) Structural monitoring: IWSNs are also used to sense the movement and material binding of the structures. A relatively higher vibration is observed, especially where large scale machinery is used. The use of sensor networks helps the engineers to monitor such structures in much detail and any deterioration in the structure, overtime, can be identified. The use of IWSNs allow better monitoring of foundations, reduced costs of regular visits, better data collection and improved data storage facilities. Monitoring of vibration and stress vulnerable structures using wireless technology helps in taking simultaneous reading of load, pressure and stress which not only accurately formulates the status of the structure under different conditions but also builds a database necessary for the future structure improvements [320], [321].

5) Disaster prevention: Extension of the industries to mining, underwater structures and critical areas where higher chances of disaster exist, need careful and time to time evaluation to identify any recently developed threats. Under such circumstances, IWSNs offer means of regular data accumulation to identify any threats present [320]. IWSNs also play key role in the mines where a certain proportion of gases is maintained and in case of any failure, actuators (suction pumps for extracting harmful gases) are instantly activated to avoid any hazards.

6) Sensor and actor networks: Wireless Sensor and Actor Networks (WSANs) offer a framework for distributed sensing and feedback based control of processes [319]. Since the wireless medium is used to establish connection between the sensors, control center and actuators therefore two data streams (Uplink:from sensors to control center and Downlink: from control center to actuators) are maintained. As more network elements are involved in such applications, more extensive and carefully modelling of the network is needed. Since the process control is implemented using sensory data therefore such networks are time sensitive and need efficient and timely data routing.
7) Industrial automation and feedback control: The incorporation of IWSNs in industrial automation brings many benefits, however the critical nature of the processes in such environments demands strict time and reliability bounds [17], [42]. Certain assurances on packet reception and reliability must also be provided for feedback control systems to operate properly. There is also a need of extensive measures to be implemented to counter the uncertainties of wireless medium of communication [343].

8) Emergency response in industries: Another emerging application area for the IWSNs is the flawless communication establishment of highly critical information. Since the consequences of delay and penalty of failure is much higher, this particular field desires a relatively flawless operation of IWSNs in terms of reliability and relative time bounds [352]. Although, IWSNs offer great potential for this area of application, however, it is still a long way from perfection.

\section{IWSN Architecture}

Over the years, a gradual rise in the implementation of IWSNs can be seen. Due to consistent research efforts during recent years, the present architecture can be characterized into several attributes. The performance of IWSNs is mainly influenced by hardware, network topology, channel access schemes, network architecture, data collection, interconnectivity, and security schemes. Therefore, the suitability of IWSNs in different applications is mainly determined from the selection and choice of these attributes. Each selection of IWSNs scheme, whether it is used for critical or noncritical applications offer some benefits but also pose certain limitations. Therefore, in application specific design, it is very important to have a careful selection of suitable attributes. Some of the key influencing factors are discussed below.

1) Nodes/Motes: Each network in IWSNs is formed with individual nodes, primarily equipped with a processing unit, radio, memory, sensor board and battery. In certain specific applications, the individual nodes may be equipped with energy harvesters, dual radios or multiple processors to offer off-the-shelf benefits [38], [90], [91]. Objective for such variations may include network lifetime extension, diversity, multithreading etc. [39], [92], [93]. A general architecture of wireless node is presented in Fig. 10, whereas a detailed discussion on wireless nodes can be found in Section VII.

2) Network Topology: In IWSN architecture, the network topology greatly influences the target application areas. Any IWSN may have a variety of network topologies with each offering a different blend of characteristics. Nodes within a network are more generally connected in star, mesh and tree topology [371]. However some other topologies including ring, bus, grid and circular are also sometime considered [371]. Some variants of the above topologies like tier1 and split-tier1 are also sometime considered [94], [95].

- Mesh topology (as represented in Fig. 4 (a)) provides better reliability and connectivity in case of larger networks but offers extended delay as a consequence of allowing multiple links to gateway and flexibility to opt most stable route for information communication [362]. 


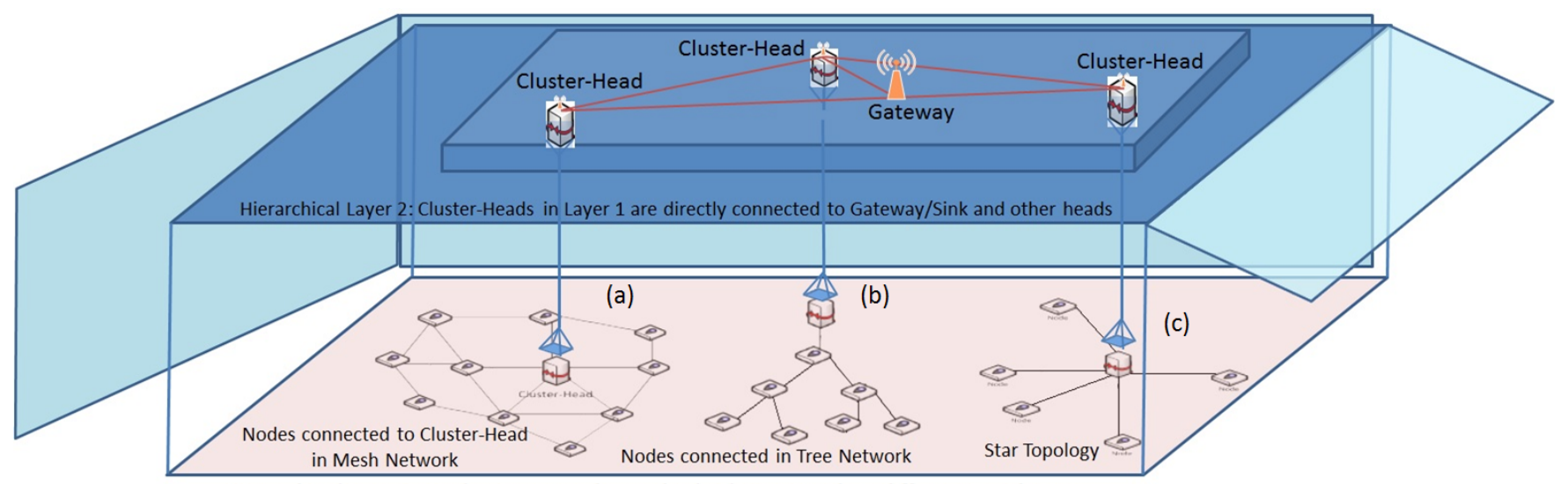

Hierarchical Layer 1: Nodes Connected to multiple Cluster-Heads in different Topologies

Fig. 4: Layered view of the Hierarchical architecture in IWSNs. Layer 1: Representation of sensor nodes affiliated with cluster-heads in a clustered network, Layer 2: Virtual representation of Cluster-heads connection with the gateway (a) Mesh Network Representation, (b) Tree Network Representation, (c) Star Topology Representation

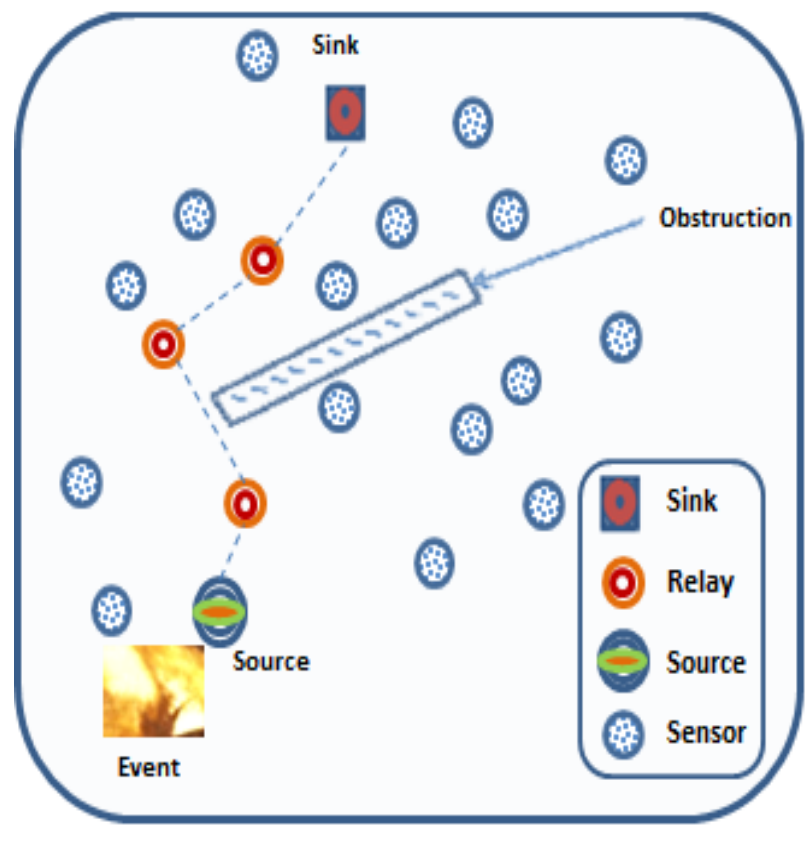

(a)

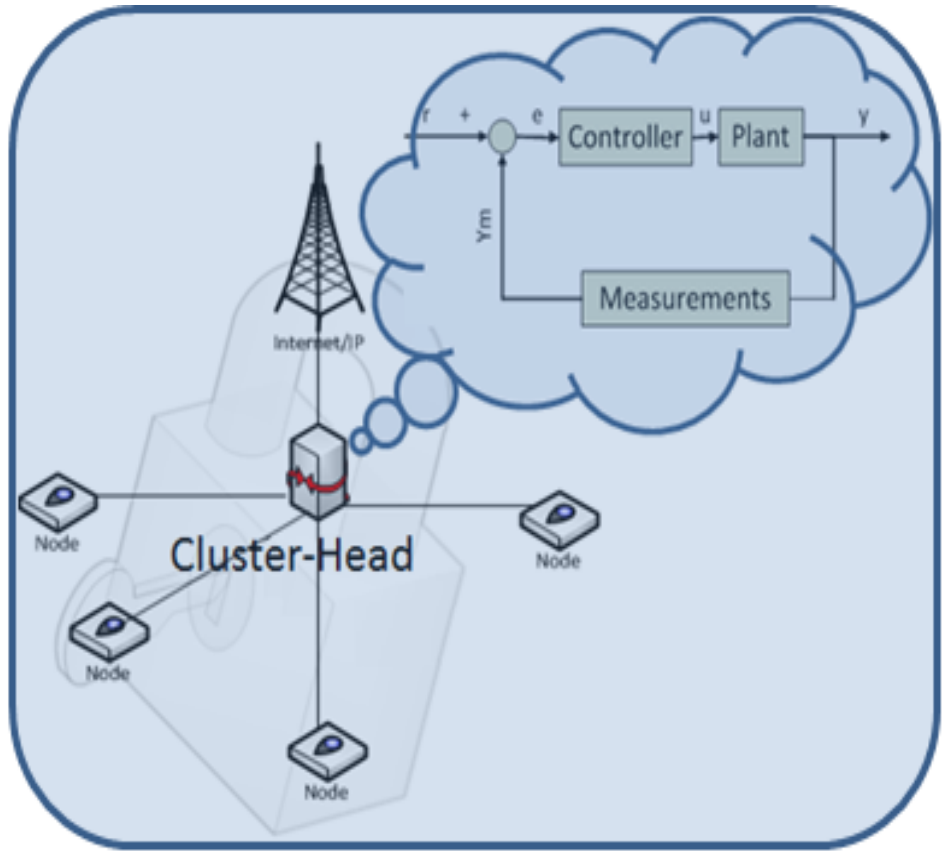

(b)

Fig. 5: Industrial Wireless Sensor Network Architecture. (a) Flat architecture with multi-hop communication to Sink, (b) Distributed control implementation with cluster-head based localized decisions for reduced delay and improved security

In this topology, each node is connected to multiple nodes which allows the networks to offer improved reliability along with self-healing abilities [363].

- Tree topology (represented in Fig. 4 (b)) offers dedicated links which allows less information overhead [364]. Each nodes communication takes fixed number of hops to reach the destination which adds deterministic behaviour to the communication. Tree topology offers gradient information field which limits the information packets straying from the path [365]. However, this topology is link dependent and failure in key linking nodes can affect large network branches. Furthermore, in time sensitive industrial applications, the use of extended branches is not feasible due to added delay.

- Star topology (represented in Fig. 4 (c)) offers direct access to the gateway which gives great improvement in the real-time data delivery however, on the other hand, reliability starts suffering with the increase in number of connected nodes especially in contention based channel access schemes[366], [367].

- Bus topology considers symmetric connection to all the nodes in the network and information is broadcasted onto the network. All nodes in the network can see all the communications but only the intended recipient receives 
the message. Bus topology is easy to install however, congestion control and security of information are major issues [368], [371].

- Ring topology forms a circular ring of nodes. Each node in the ring topology is connected to exactly two nodes where each communication in the ring can either be clockwise or anti-clockwise [369], [371]. The ring increases the chances of failure as a disconnection in the ring can result in the failure of entire system. Furthermore, the security and congestion issues are more prominent.

- Circular topology formulates circular sensing area with sink at the centre. The nodes are deployed with uniform density and network can span a large area. The topology can accommodate widespread sensing nodes capable of forming multi-hop network. The communication from the sensor nodes to the sink can be single-hop or multi-hop depending on the sensing node's distance from the sink and transmission range. The circular topology is easy to establish and maintain however, geographical attributes are hard to find in industrial environment [370], [371].

- Grid topology partitions the network into nonoverlapping square grids of the same size. Each grid accommodates one working node at a time. For grids with multiple nodes, the grid activity is handled by gridhead which collects data from the grid. The routing in this topology is performed in grid by grid manner [371], [372].

3) Channel access schemes: In IWSNs two channel access schemes, TDMA and CSMA/CA, derived from IEEE 802.15.4 [23] and IEEE 802.15.4e [20] standards are commonly used. In TDMA based channel access, the nodes follow a time slotted access for data communication. The nodes are synchronized using synchronization beacons and each node is scheduled to communicate in a pre-specified time-slot [20], [200]. In this way, a guaranteed channel access is ensured. The TDMA based channel access is well suited for periodic communications needed in regulatory control and open loop control. However, TDMA poses limits to the instant communication ability of a node with the gateway.

In CSMA/CA based channel access, the opportunistic communication is established where depending on availability of channel, a node attempts to communicate [23]. Since no dedicated bandwidth is specified for a node, a guaranteed channel access cannot be ensured. CSMA/CA based schemes also suffer from reliability issues as the number of connected nodes are increased.

Hybrid channel access schemes are also introduced where the contention based (CSMA/CA) and slotted (TDMA) channel access schemes are adaptively used, to improve overall performance of the network [10], [236], [239].

The details of TDMA based channel access schemes are presented in Section VI-B(2) and Section VIII-A(2), a detailed discussion regarding CSMA/CA based access schemes and standards can be found in Section VI-B(1) and Section VIII$\mathrm{A}(1)$ whereas the discussion related to hybrid channel access schemes is further continued in Section VIII.
4) Network architecture: Network architecture also serves as a decisive factor in performance of IWSNs. Network architecture may be flat or hierarchical, but the choice is mainly influenced by the requirements of the application. The following discussion summarizes the key features of each architecture type.

- Flat architecture offers traditional benefits of low complexity and is suitable for small networks. However, as the network starts to expand, the delay starts becoming unbearable. Furthermore, to handle the multihop communication, the control overhead and data relay path selection information in term of routing tables and other path selection mechanism overstress the network. In addition to this, in flat architecture certain nodes become stress points or bottle neck for the network performance. A representation of flat architecture is shown in Fig. 5(a).

- Hierarchical architecture on the other hand offer quick access to the critical information and keeps the clusters small enough to avoid traffic overload. On the down side, hierarchical architecture adds extra complexity where each node must be affiliated with suitable cluster head and failure in doing so may induce longer time delays and compromised reliability. A hierarchical architecture is represented in Fig. 4, where layered view is used to present the connectivity of the nodes. As it can be seen in the figure, at layer 1, the sensor nodes are connected to cluster-head and information from the nodes is collected at cluster-head in timely fashion. Layer 2 represents the upper hierarchy of connections in the hierarchical architecture, where the cluster-heads are connected to the control center/Gateway. The communication in the represented scenario is a two step process where in the first step the sensor data is accumulated at the clusterhead whereas in second step the accumulated date is forwarded to gateway/control center.

5) Security: IWSNs inherently possess security issues and due to its wireless nature, the regular wired network security protocols do not offer sufficient protection desired in such networks [356]. IWSNs are vulnerable to malicious attacks such as information tampering, interference, flooding, denial of service and eavesdropping [37], [42], [357]. It is for the same reason, security is considered as a mandatory attribute of industrial protocols and standards. To address the security threats, multilayer security functions are introduced. To improve the security in IWSNs countermeasures such as data encryption, cryptographic key establishment, frame protection, key rotation and device management are implemented to ensure data integrity and industry grade security [19], [40], [354].

The higher stakes in industry encourages use of multilayer security. It is for the same reason security is an integral part of well-known industrial standards for IWSNs. Security related features of some of the well-known industrial protocols and standards are listed as follows whereas a more detailed discussion on these standards is presented later in Section VIC.

WirelessHART offers a secure networking solution where 
security is implemented in both MAC and Network Layers. Message Integrity Check (MIC)is implemented to ensure hop by hop data integrity whereas end to end data integrity is ensured at network layer. Security is ensured by security manager application which handles security services. It is also responsible for generation of security keys. WirelessHART also uses 128-bit AES encryption for added security [19], [82]. In ISA100.11a, security manager is implemented which is responsible for authentication, generation, distribution and storage of the security keys. ISA100.11a also ensures end to end security [82]. The security features in ISA100.11a are similar to WirelessHART, however, unlike WirelessHART, security features in ISA100.11a are optional and can be disabled to offer more flexibility, improve overall network efficiency and boost battery life where security is not mandatory.

In Zigbee, different levels of security are implemented. To offer a secure communication, frame integrity check is performed. Key based entity authentication is also implemented to ensure the authenticity of the nodes. On the network layer, active network key is implemented to offer network layer authentication [358]. Further to aforementioned features, symmetric cipher is used to protect data from unauthorized access [358]. Like ISA100.11a and WirelessHART, Zigbee defines Trust Center which serves the purpose of a security manager.

In 6LoWPAN, Time Slotted Channel Hopping (TSCH) is implemented for efficient multi-hop communications in IWSNs. With security feature enabled in frame control, 6LoWPAN additionally adds security control, frame counter and key identifier for secure communication and protection from replay attacks. Furthermore, a 32, 64 or 128 bits Message Integrity Code (MIC) is used to enable encryption of data [359]. On the network layer, to provide integrity, authenticity, and confidentiality for IP datagrams, Authentication Header (AH) and Encapsulating Security Payloads (ESP) are added [359].

6) Data collection: In the industrial environments, the stable operation of the processes is dependent on the effective communication of sensory data to control centre. Depending on the wide variety of applications in industrial environments, the data collection mechanism can change. The communication for data collection in the industrial environments can be divided in three categories, namely periodic, event-based and on-demand communication, where each of these categories target a particular type of industrial system.

In periodic communication mode, the data is communicated in regular intervals, handled by TDMA or slotted channel access, ensured for the efficient process control [20], [212]. Periodic data communication is most suitable for regulatory control system and open loop control systems. Furthermore, some of the monitoring systems also implement loosely bounded periodic data communication tolerant to time variations in delivery of information. The periodic communication is scheduled at the start of the network and the cycle is repeated by sensor nodes which are responsible to transmit data regularly [360].

The event-based communication depends on the communication of data that takes into consideration the critical circumstances and sends data in case of occurrence of an event [360]. In industrial applications, the event can be programmed as a violation of a particular threshold or occurrence of emergency circumstances, which need to be reported. This type of data communication is usually preferred in emergency systems [352] and alerting systems. The supervisory control systems also use event-based triggers to initiate communication of sensory data in case of violation of a threshold; however, in this case the communication is established for longer duration with periodic feedback.

The on-demand communication is established in cases where the communication from a particular sensor node is requested by the coordinator or sink [361]. This can occur if the sensor data from particular node or a system is desired or a particular node communication has failed, therefore, a request for transmission of data can be generated.

7) Interconnectivity: A variety of applications in IWSNs may need a suitable interconnectivity mechanism with the existing fieldbus and Ethernet standards. Moreover, certain applications may need access to IP and the Internet. These factors definitely steer the choice of suitable industrial solutions. Although, Zigbee, 6LoWPAN and wirelessHART are some of the examples offering distinct blend of interconnectivity options in wide range of automation and supervisory control industry [40], yet the connectivity to fieldbus elements is still a challenge.

\section{E. Congestion in IWSNs}

In most IWSN applications, communication is bounded by strict time and reliability constraints. These strict bounds make IWSNs more vulnerable to congestion problems. In network communications, congestion causes considerable delay, packet loss, energy wastage and uncertainty. The congestion is mostly attributed to un-coordinated transmission of information, simultaneous data reporting and buffer overflow [442]. To overcome the problems of congestion various researches have been documented in the literature.

Congestion in the network is minimized using congestion control policies and congestion control mechanisms. Congestion control policies are usually termed as open-loop congestion control as these policies dictate certain rules regarding retransmission of packets, packet acknowledgments and discarding packets [443]. The effective implementation of congestion control policies reduces the overall network overhead and limit excessive transmissions in the network. However, the policies cannot ensure run-time congestion minimization. To address the dynamic congestion issues and to minimize congestion adaptively, congestion control mechanisms are defined. Congestion control mechanisms use congestion detection like channel load, buffer overflow etc. to detect and locate congestion in IWSNs [444]. With the use of TDMA based communication in IWSNs, the congestion from neighbouring nodes is significantly reduced. However, due to the limited capacity of the network, the packet in-flow from the upstream nodes may be exceeding the threshold. In such cases, congestion control mechanisms implement congestion notification for upstream nodes to counter the congestion problem [445].

Different congestion detection techniques and congestion notification mechanisms are presented in Fig. 6. The figure also 


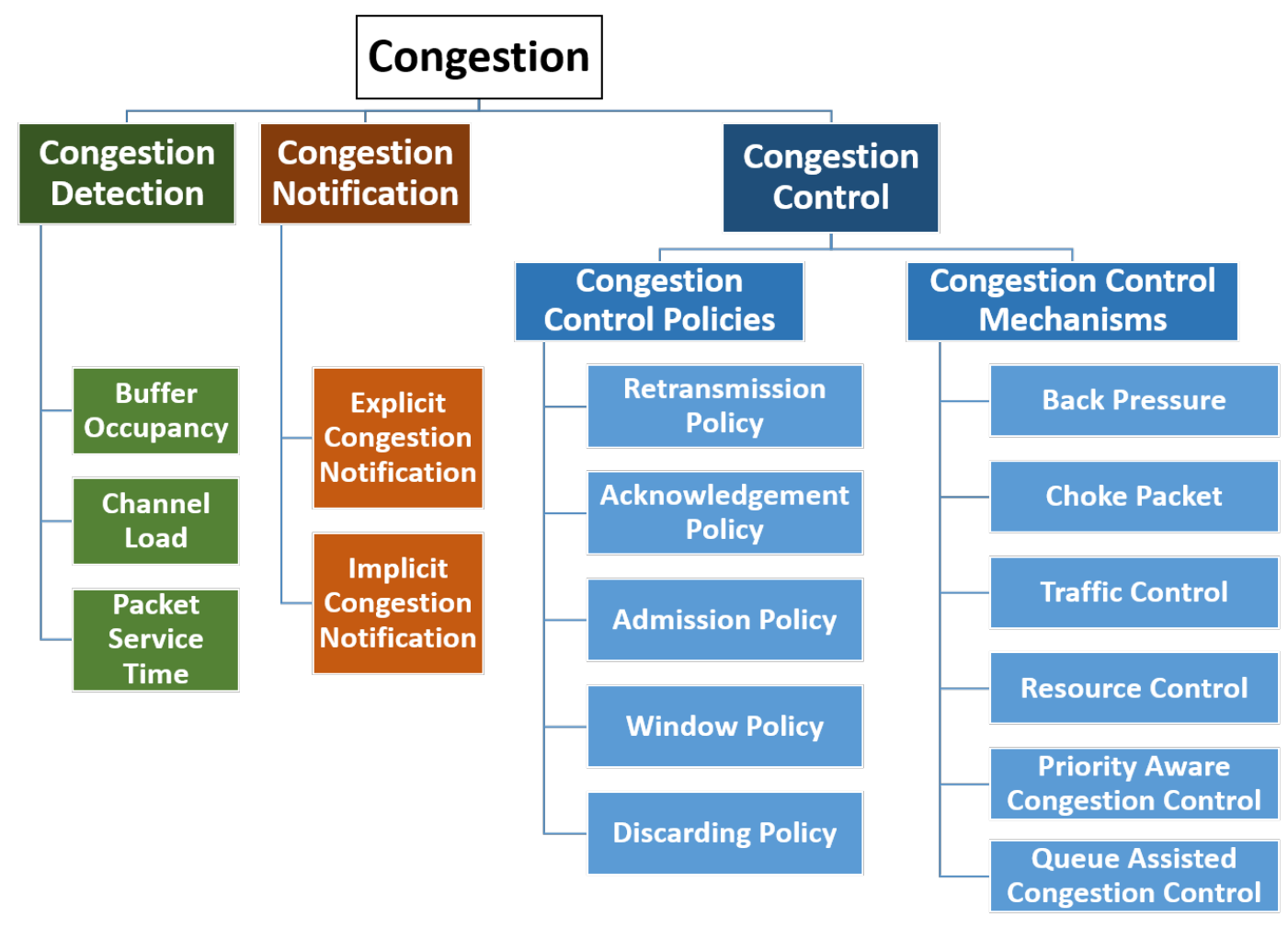

Fig. 6: Congestion in IWSNs

presents congestion control policies and congestion control mechanisms to address the congestion issues in IWSNs. Further discussion regarding these categories and congestion control on the whole, can be found in [444], [443], [445], [442], [446] and [447].

\section{IWSNS DESIGN OBJeCtives AND CHALLENGES}

The development in IWSNs over the years is influenced by certain design objectives. However, the achievement of these design objectives is affected by certain limitations of IWSNs. The primary design objectives and possible design challenges in IWSNs are listed as follows.

\section{A. Design Goals and Objectives}

In IWSNs, continuous research has provided much wanted improvements in past few years. It is because of the efforts of many individuals and some joint ventures that IWSNs have recently witnessed much wider acceptability in several industrial applications. Due to a broad scope of the potential applications of IWSNs and with rising challenges, certain design objectives need to be considered.

Wireless links in industrial automation, whether it is for emergency communication, process control, feedback systems, altering or monitoring networks, requires certain performance and reliability assurances. To fulfil these requirements using IWSNs, certain design goals and objectives must be set forth. Although, design objectives are very much linked to individual applications, their requirements, and targeted industrial systems, yet the design goals and objectives for different cases are listed as under.
- Efficient resource (battery, processing power, memory, bandwidth etc.) usage

- Ensuring predictable delay and latencies (Mandatory condition for effective regulatory and supervisory control)

- Managing congestion free communication

- Accurate time synchronization

- Real-time assurance

- Security of information

- Modular design for improved scalability

- Interoperability with existing infrastructure

- Prioritized communication

The above-listed objectives offer best practices for extended network life time, reliability of communication, an appropriate priori information of delay for efficient regulatory and supervisory control, a scalable network offering modular design for more flexibility, improved security features, jitter minimization, surety of real-time communication for sensitive processes and proper priority affiliation to different traffic types for timely and precedence based communication. The design goals, research developments and limitation in achieving these design objectives are presented in Table IV. Whereas, certain other challenges offering notable deterrent in achieving these objectives are listed in Section V-B.

\section{B. Design Challenges}

The collaborative nature of IWSNs enables this technology to bring many off the shelf advantages, however, there are certain bounds to the benefits offered by IWSNs especially when coping with harsh environments. These bounds give rise 
to certain research challenges as well. The major challenges in the present IWSNs are as under.

1) Resource constraint: IWSNs do serve as a cost-effective and efficient solution to the industrial automation and process control. Although, the low cost and reduced complexity of the IWSNs offer a suitable solution for the industrial applications yet it also adds cap to the resources of the tiny wireless motes [17], [19], [50]. Resource constraints in IWSNs can be divided into six different sections as below. 
TABLE IV: Goals and Objectives: limitations and research developments

\begin{tabular}{|c|c|c|c|}
\hline $\begin{array}{l}\text { Goals and } \\
\text { Objectives }\end{array}$ & Aspects & $\begin{array}{l}\text { Progress and } \\
\text { Research S- } \\
\text { tatus }\end{array}$ & Description \\
\hline \multirow{4}{*}{$\begin{array}{l}\text { Efficient } \\
\text { Resource } \\
\text { Usage }\end{array}$} & Battery & $\begin{array}{l}\text { Partially } \\
\text { addressed }\end{array}$ & $\begin{array}{l}\text { Limitations } \\
\text { 1) Limited battery capacity [323], [35], [39] } \\
\text { 2) Capacity increase at the cost of size, weight, and price [16] } \\
\text { 3) Decrease in the battery capacity with time and recharge cycles [35], [39] } \\
\text { Research developments and improvements } \\
\text { 1) Energy efficient utilization of IWSNs } \\
\text { - Sleep scheduling [324], [325], [326], [327] } \\
\text { - Transmission power control [104], [105], [106], [107] } \\
\text { - Deep sleep and passive listening [324], [328], [329] } \\
\text { - Data redundancy reduction [118], [330] } \\
\text { 2) Use of rechargeable batteries with energy harvesting for extended unsu- } \\
\text { pervised operation [35], [39] }\end{array}$ \\
\hline & $\begin{array}{l}\text { Processing } \\
\text { power }\end{array}$ & Addressed & $\begin{array}{l}\text { 1) Wide variety of microcontroller with diverse range of processing capa- } \\
\text { bilities suitable for IWSNs [331], [332] } \\
\text { 2) Tremendous reduction in cost factor (Use of SuperSRAM and DRAM) } \\
\text { [333] }\end{array}$ \\
\hline & Memory & $\begin{array}{l}\text { Partially } \\
\text { addressed }\end{array}$ & $\begin{array}{l}\text { Limitations } \\
\text { 1) Limited memory capacity in the available wireless nodes and microcon- } \\
\text { trollers [334], [335] } \\
\text { 2) Limited code memory, restricting implementation of complex algorithms. } \\
\text { [100] } \\
\text { Possible solutions and remedies } \\
\text { 1) Introduction of more powerful nodes with extended memory [169], [336] } \\
\text { 2) Optimized memory utilization [100], [337] }\end{array}$ \\
\hline & $\begin{array}{l}\text { Bandwidth } \\
\text { and } \\
\text { transmission }\end{array}$ & $\begin{array}{l}\text { Partially } \\
\text { addressed }\end{array}$ & $\begin{array}{l}\text { Limitations } \\
\text { 1) Overlapping spectrum in IWSNs [98], [338] } \\
\text { 2) Possible interference due to overlapping channels in WiFi, WPAN and } \\
\text { other technologies operating in ISM band [143], [301] } \\
\text { Research developments and improvements } \\
\text { 1) Cognitive channel access for interference avoidance [305], [308] } \\
\text { 2) Adaptive channel selection and contention free channel access [339] }\end{array}$ \\
\hline $\begin{array}{l}\text { Predictable } \\
\text { Delay and } \\
\text { Latencies }\end{array}$ & $\begin{array}{l}\text { Delay and } \\
\text { Deadlines } \\
\text { with } \\
\text { predictable } \\
\text { latency }\end{array}$ & $\begin{array}{l}\text { Partially } \\
\text { addressed }\end{array}$ & $\begin{array}{l}\text { Limitations } \\
\text { 1) Hard deadlines [42], [53] } \\
\text { 2) Unpredictable delays in dense networks [340] } \\
\text { 3) Unsynchronized deadlines and asynchronous channel access requirements } \\
\text { from emergency and supervisory control.[51], [53] } \\
\text { Research developments and improvements } \\
\text { 1) Segmented slot access and retransmissions [20], [82], [95], [138] } \\
\text { 2) Priority based communication [55], [218], [219] } \\
\text { 3) Asynchronous communication scheduling protocols for emergency com- } \\
\text { munication [149] } \\
\text { 4) QoS assurance [11], [31], [305] }\end{array}$ \\
\hline
\end{tabular}




\begin{tabular}{|c|c|c|c|}
\hline $\begin{array}{l}\text { Congestion } \\
\text { free com- } \\
\text { munication }\end{array}$ & $\begin{array}{l}\text { CSMA/CA } \\
\text { schemes }\end{array}$ & $\begin{array}{l}\text { Partially } \\
\text { addressed }\end{array}$ & $\begin{array}{l}\text { Limitations } \\
\text { 1) Random channel access [209], [210], [225] } \\
\text { 2) Interference [98] } \\
\text { 3) Lack of scheduling of communications [214], [215], [217] } \\
\text { 4) Back-off based delay in CSMA/CA based access schemes [340] } \\
\text { Research developments and improvements } \\
\text { 1) Channel estimation [341], [342] } \\
\text { 2) Kalman based and other congestion control algorithms [343], [344] } \\
\text { 3) Use of slotted CSMA/CA schemes [211], [212] }\end{array}$ \\
\hline & $\begin{array}{l}\text { TDMA } \\
\text { schemes }\end{array}$ & Addressed & $\begin{array}{l}\text { 1) Use of TDMA for interference free communication [20], [200] } \\
\text { 2) Guaranteed channel access [20], [201], [202] }\end{array}$ \\
\hline $\begin{array}{l}\text { Time } \\
\text { synchro- } \\
\text { nization }\end{array}$ & $\begin{array}{l}\text { Single-hop } \\
\text { topology }\end{array}$ & Addressed & $\begin{array}{l}\text { 1) Use of beacon enabled communication for nodes synchronization [20] } \\
\text { 2) Synchronization with the master clock. [20], [345] } \\
\text { 3) Ensuring recalibration of the faulty (lagging, leading) clocks [345], [346] }\end{array}$ \\
\hline $\begin{array}{l}\text { Time } \\
\text { synchro- } \\
\text { nization }\end{array}$ & $\begin{array}{l}\text { Multi-hop } \\
\text { networks }\end{array}$ & $\begin{array}{l}\text { Partially } \\
\text { addressed }\end{array}$ & $\begin{array}{l}\text { Limitations } \\
\text { 1) Large networks with multi-hop communication [345] } \\
\text { 2) Non-linear processing and communication delays. [347] } \\
\text { 3) Usage of low cost and less accurate clocks } \\
\text { Research developments and improvements } \\
\text { 1) Consensus based time synchronization: Average Time Synchronization } \\
\text { (ATS) and Maximum Time Synchronization (MTS) [348] } \\
\text { 2) Post facto synchronization and tunable synchronization [349] } \\
\text { 3) Source clock frequency recovery (SCFR) based and distributed time } \\
\text { synchronization [350], [351] }\end{array}$ \\
\hline \multirow[t]{2}{*}{$\begin{array}{l}\text { Real-time } \\
\text { assurance }\end{array}$} & Emergency & $\begin{array}{l}\text { Partially } \\
\text { addressed }\end{array}$ & $\begin{array}{l}\text { Limitations } \\
\text { 1) Asynchronous access requirements [352] } \\
\text { 2) Effects of TDMA induced access delay on instant channel access require- } \\
\text { ments [352] } \\
\text { Research developments and improvements } \\
\text { 1) Multichannel solution [203], [223], [224] } \\
\text { 2) Priority based channel access execution [55], [218], [219] } \\
\text { 3) Maximum delay assurance [20] }\end{array}$ \\
\hline & $\begin{array}{l}\text { Process con- } \\
\text { trol }\end{array}$ & $\begin{array}{l}\text { Partially } \\
\text { addressed }\end{array}$ & $\begin{array}{l}\text { Limitations } \\
\text { 1) Periodic channel access requirements [51], [55] } \\
\text { 2) Deadlines in milliseconds [42], [53] } \\
\text { 3) Lack of appropriate remedies in case of communication failure [20], [235] } \\
\text { Research developments and improvements } \\
\text { 1) Priority based access [55], [218], [352] } \\
\text { 2) Shared slots for retransmission of critical communication [20] } \\
\text { 3) On-demand channel access [352] } \\
\text { 4) Deadline optimized communications [20], [55] }\end{array}$ \\
\hline
\end{tabular}




\begin{tabular}{|c|c|c|c|}
\hline Security & $\begin{array}{l}\text { Security } \\
\text { threats, } \\
\text { integrity and } \\
\text { authenticity }\end{array}$ & $\begin{array}{l}\text { Partially } \\
\text { addressed }\end{array}$ & $\begin{array}{l}\text { Limitations } \\
\text { 1) Information tampering [37], [353] } \\
\text { 2) Node control [37], [42] } \\
\text { 3) Denial of service [37], [42] } \\
\text { 4) flooding [37], [42], [353] } \\
\text { 5) radio interference [37], [42], [353] } \\
\text { Research developments and improvements } \\
\text { 1) Hop by hop data integrity [19] } \\
\text { 2) Data encryption [19], [40] } \\
\text { 3) Cryptographic key establishment [40], [354] } \\
\text { 4) Frame protection and device management [40], [354] }\end{array}$ \\
\hline $\begin{array}{l}\text { Interoper- } \\
\text { ability }\end{array}$ & $\begin{array}{l}\text { WPAN } \\
\text { to Other } \\
\text { mediums }\end{array}$ & $\begin{array}{l}\text { Partially } \\
\text { addressed }\end{array}$ & $\begin{array}{l}\text { Limitations } \\
\text { 1) Lack of standardization [16] } \\
\text { 2) Integration with existing systems [19] } \\
\text { 3) Too many technologies in play [19] } \\
\text { Research developments and improvements } \\
\text { 1) Multi-interface motes [152], [153], [162] } \\
\text { 2) Interoperability with the IPv6 and other networks [28] }\end{array}$ \\
\hline $\begin{array}{l}\text { Prioritized } \\
\text { communi- } \\
\text { cation }\end{array}$ & Static/Dynamic & $\begin{array}{l}\text { Partially } \\
\text { addressed }\end{array}$ & $\begin{array}{l}\text { Limitations } \\
\text { 1) Priority Establishment } \\
\text { 2) Attributes of priority [55], [218] } \\
\text { 3) When and how to be prioritized [219] } \\
\text { 4) Static priority systems [55] } \\
\text { Research developments and contributions } \\
\text { 1) Introduction of priority systems [51], [55] } \\
\text { 2) Optimization of protocols w.r.t to channel access and priority of service } \\
\text { [53], [55], [352]. } \\
\text { 3) Redefinition of usage of shared slots and asynchronous channel access } \\
\text { [10] } \\
\text { 4) Hybrid channel access schemes for improved flexibility [53] } \\
\text { 5) Multichannel schemes for improved performance [29], [53] }\end{array}$ \\
\hline
\end{tabular}

- Battery: IWSNs are equipped with limited battery. The battery issue is even more critical where the motes are inaccessible due to critical nature of the application. For instance, thermal analysis of installed machines and interior sensor placement in large synchronous generation units need long lasting battery to make the most of these inaccessible motes. For most of the cases in IWSNs the motes are designed to last longer with the inbuilt ability to preserve battery where possible. This property especially helps in applications where replacing a mote is more effective than replacing battery of an existing mote. However, not much improvements can be seen in enhancing the existing energy sources (Lithium-Ion, Alkaline, Zinc-air etc.), thus, leaving the only solution to increase the size of the battery which is not an efficient alternate. Hence the battery capacity becomes a major bottle neck for most of the cases where motes cannot be used to full potential to achieve extended features [96], [97].
- Communication range: IWSNs suffer from limited range especially in harsh industrial environments. The communication range for wireless sensor motes can extend from $10 \mathrm{~m}$ to $300 \mathrm{~m}$ depending on the severity of reflection, refraction, diffraction, multipath, interference and fading [142], [153], [173]. The short range, where at one instance adds to the lifetime of the network, it also affects the real-time delivery of the data to destination. Hence, gives rise to the need of efficient routing algorithms in multi-hop communication.

- Bandwidth: For the IWSNs, a dedicated band specified for industrial, scientific and medical (ISM) applications is used. This band is reserved internationally for the use of radio frequency (RF) energy for industrial, scientific and medical purposes. Where this dedicated band provides scope for low cost industrial communication, it also limits the band access as well. Most of the radios available, 
operate at $2.4 \mathrm{GHz}$, with an accessible frequency bandwidth of $73 \mathrm{MHz}$. This frequency bandwidth is divided in eleven channels [98]. The technology offers a data rate of $250 \mathrm{kbps}$ which, for some of the applications in industrial domain, serve as a bottleneck. The industrial applications using $868 \mathrm{MHz}$ and $915 \mathrm{MHz}$ instead of $2.4 \mathrm{GHz}$ suffer even lower data rates [98].

- Memory storage: Limited memory in IWSNs offers a major challenge [99], [100]. Even with some of the latest motes in market, memory still remains a constraint. Though increase in memory is not one of the tough tasks yet it comes with an additional cost, especially for the applications requiring a large number of motes. With the limited memory capacity, the scope of more sophisticated algorithms is restricted [100], [101]. A more efficient memory allocation and optimized memory utilization is expected to improve most of the existing technology without additional cost.

- Processing capability: The rapid improvements in the Micro Electro Mechanical Systems (MEMS) enable the present-day chips, the processing capability of several hundreds of $\mathrm{MHz}$ at a relatively low cost. But in IWSNs low clocks are preferred. Only reason that justifies this adoption in the field of IWSNs is the fact that with high processing capabilities, the chip becomes more power hungry and results in early depletion of the battery. To avoid such high power consumption and to extend the network life, the motes are mostly low clocked and hence offer ability to run only low complexity algorithms [100].

- Half duplex communication: Till present, almost all of the motes available in the market offer half duplex communication [102], [103]. This attribute of the motes, used for industrial applications, adds need to use cooperative nature of communication along with better synchronization. Certain mechanisms have been proposed to overcome the half duplex nature of the available motes like CSMA/CA with exponential back off but still it poses serious limitations to the practical use of motes.

2) Energy consumption and network lifetime: As discussed earlier, battery capacity per unit volume remains almost static over the years. some improvements presented over time are either restrained by cost factor or instability of battery material in industrial atmosphere. Therefore, the smart energy consumption and means to efficiently optimize the energy usage of the motes has been thoroughly investigated. However, while the energy efficiency is achieved using transmission power control, energy efficient routing, sleep scheduling, or localized processing, there is no benchmarking and most of the accomplished work is questionable due to lack of its authenticity in harsh environments. Since the use of IWSNs in some inaccessible industrial environment signifies the need of efficient energy utilization to offer extended network lifetime to cope with the long-lasting lifetime of the industrial equipment, a thorough evaluation of existing research in stringent industrial environments is much needed.

3) Time Constraint: Time deadlines are one of the most critical parameters in an industrial automation and process control, where slight delays can cause severe damages to the equipment, spoil an entire lot under production, or can even cause threat to human life. Delay can be even more critical for sensitive processes under observation like nuclear plants. In any industrial setup, a whole system is devised to extract the critical information from various individual processes and establish a control system to make critical runtime decisions. For all close loop control systems, a particular time deadline is of imminent importance and functionality of the control system depends on the real-time delivery of this information. In IWSNs the real-time data delivery appears to be a major challenge due to limited range and susceptibility to the harsh industrial interference, humidity, multipath, dust and highly caustic environments [16]. Therefore, if the IWSNs are opted as an alternate for wired networks this aspect must be thoroughly evaluated and verified.

4) Interference, reliability, and bit error rate: In any industrial environment, interference is one of the inherited problems and usually appears as a major challenge in IWSNs. Interference is relatively high in industrial setups due to high noise, electromagnetic radiations, multipath distortion, humidity, dust and dynamic atmosphere. All these factors contribute to relatively reduced range, distorted and noisy transmission, frequency selective fading and non-reliable links, eventually resulting in extended packet delay and high packet loss ratio. To offer a reliable communication without violating critical deadlines, the impact of interference cannot be ignored. Transmission power control [107] and optimal communication power adjustment algorithms along with certain other strategies can be considered as a source of contention and interference avoidance within the local area networks [104], [105], [106], none the less the issue needs to be addressed properly.

For more critical processes, the data received from different carefully placed sensors to sample the critical information serves as a marker to identify the stability of the process. Moreover, the CSMA/CA based channel sensing and collision avoidance schemes can ensure the reliability up to certain extent but with the increased traffic, the reliability starts to suffer [108], [109]. Also some parameters including the Link Quality Indicator (LQI) and Radio Signal Strength Indicator (RSSI) are considered as the identifiers for the link quality and reliability yet they may be deceptive [110]. Bit error rate, on the other hand offers more reliable evaluation of performance of the communication in digital wireless link. However, achieving a bit error rate of the order supported by the wired networks is a major challenge due to uncertainty in wireless channels. Usually the bit error rate is carefully defined based on the needs of the process along with acceptable levels of error. 
5) Variable link capacity: IWSNs operate at relatively low data rate with an upper cap of $250 \mathrm{kbps}$ with only few exceptional high data rate schemes. The achievable data rates in reality are relatively less due to congestion, interference, high distortion noise, multipath distortion and synchronization overhead. Moreover, contrary to general assumptions, the link capacity is not constant rather it depends on the channel conditions. The changing link capacity forces the researchers to model the link with ability to provide connectivity between source and destination with certain traffic carrying capacity along with the implementation of counter measures for the imminent uncertainty in wireless links.

6) Synchronization: The evolution of IWSNs for industrial applications enforced the need of reliable data communication. It was the basic reason why most of the industrial applications of WSNs have seen a dramatic shift from CSMA/CA based channel access schemes to Time Division Multiple Access (TDMA). The use of TDMA ensured a higher percentage of contention free channel access to the source/sensor nodes in IWSNs. However, it also gives rise to the need of proper synchronization among the wireless motes in the network.

7) Sleep Scheduling: With TDMA in place, efficient sleep scheduling algorithms can exponentially enhance the lifetime of the network. At the same time, for any industrial plant, information has great significance and missing critical information can be catastrophic. Hence, advanced mechanisms are needed to provide a proper scheduling with alternate paths in place to carry critical information. Moreover, other mechanisms must be exploited to offer extended network lifetime without undermining the performance of the network. Though a great contribution in this aspect has been witnessed [111], [112], [113], [114], [115], [116], [117], yet the sleep scheduling for hybrid access schemes are not much optimized and though these schemes offer better performance yet fail to provide good energy efficiency and sleep scheduling [10].

8) Data redundancy: In IWSNs, data redundancy is usually exploited to achieve better reliability. However, beyond certain bounds, the redundancy becomes a source of quality degradation. Mostly the redundancy is modeled as a static aspect of a protocol [118], [119]; however, there is a need to treat redundancy as a dynamic aspect, which is directly linked to communication overhead and link capacity.

9) Scalability: Scalability is one of the most important attributes of the IWSNs. The emphasis on this aspect is even greater for industrial applications due to the ever-changing industrial environment. Although the use of IWSNs as a solution for industrial processes inherently offers flexibility to the changes in the existing processes yet the scalability stands as a major hurdle. It is observed that an increase in the network size results in computational and communication overhead, which becomes unbearable for the wireless nodes and hence the process fails to cope with the increased demand.

10) Network topology: Careful selection of network topology is very important as it has notable impact on the intrinsic properties and performance of the network. In literature, many topologies were listed with their benefits and weaknesses. Some of these topologies include star, mesh, tree, circular, grid, ring, bus, tier-1 network topology, split tier-1 network topology and linear topology [371]. In some cases, star topology (extended as multi directional split tier-1 topology) and hierarchical topology (a multi-level star topology) were considered. Some adaptive and static schemes were introduced to improve the performance of industrial wireless networks [120], [121]. It is known that all these topologies have certain effects on the various performance metrics including the reliability, real-time data delivery, bit rate and complexity. However, to establish a direct relationship of performance metrics with a topology under investigation, still needs extensive evaluation.

11) Security: IWSNs, whether used in critical or noncritical industrial application, is always vulnerable to security threats [122], [123]. The security threats may be active or passive. To overcome the threats of the external interference and to block malicious information, the need for sophisticated encoding schemes and malicious information identifiers is inevitable. Moreover, some hardware based security schemes [124] may assist in ensuring better security features for industrial applications.

12) Coexistence: In the past few years significant improvements in the existing structure of IWSNs have been witnessed. In all these years with various researches carried out, different domains of IWSNs were targeted. From all the significant improvements whether they included network layer, MAC layer or PHY layer, the only attribute of these researches remained ambiguous was the coexistence of these improvements. Although these improvements in different layers and aspects of IWSNs emerged in form of standardization [20], [82], [140] , however, still the there is much room for improvement. For all the algorithms defined, whether they target routing, MAC restructuring, sleep scheduling, transmission power control or any other, the evaluation of collective enhancement of these achievements is still a challenge [375].

13) Information priority scheduling: All the information in industrial applications can be divided into multiple categories based on the critical nature of the information. It has always been a challenge to optimize the flow of information and prioritize the critical information due to the existing MAC layer structure in the baseline standards. Some scheduling algorithms were proposed [10], [30], [55] to prioritize the information but priority scheduling still remains a challenge in an industrial atmosphere.

14) Contention free medium access: Contention free medium access remains one of the most considerable attributes in any industrial environment. This served as a primary reason for the changes in the industrial standards for WPAN from CSMA/CA to TDMA. With the use of TDMA, current standards offer guaranteed access to the medium in the specified slots. However, the problem is not completely resolved as retransmissions still use contention based channel access schemes. For retransmission, the latest industrial standards use shared slots [20], with contention based access to the slot. All the sources with lost packets use CSMA/CA based scheme with exponential back off to gain access to the shared slot, which once again pose challenge of maintaining collision free medium access [20].

15) Energy harvesting: In the past few years, no significant improvements in the existing batteries have been made. For 
longer network lifetime, the solution emerges from either bigger battery attachment or efficient battery utilization. One other trend has also been seen where the energy harvesting schemes are used to offer extended network lifetime. However, the energy harvesting techniques in IWSNs need further evaluation.

16) Mote size and protective casing: With the recent development in the fabrication of sophisticated printed circuit boards and nano-technology at hand, the size of the motes can be significantly reduced, yet the battery remains a major contributor in size of the mote. Moreover, the energy harvesting schemes can also add to the size of the mote. With certain frequency bands $(868 \mathrm{MHz}$ and $915 \mathrm{MHz})$ still in use for industrial automation and process control, the antenna size also contributes significantly in increased mote size and dimensions.

Apart from this, the harsh industrial environments encourage the use of suitable protective casing for sensitive equipment. Any equipment used in the industry is needed to be tested for the critical conditions, that it will go through during its operation on an industrial floor. Same is the case with the wireless sensor motes, which need to survive in harsh industrial environments. Any design of casing for these motes may need to withstand, hot and cold climate, excessive heat, rapid temperature fluctuations, corrosion, humidity, fumes, fungus, vibration, pressure and radiations. Hence, design of casing for the wireless motes has great significance and must not be considered lightly. Unfortunately, this particular area lacks substantial research to assist in low weight, low cost and reliable casings for harsh environments.

17) Multi-hop communication: One of the primary reasons for opting IWSNs for industrial applications was the inherited ability of these networks to establish multi-hop communication. This feature of IWSNs permits the flexibility to add new sources and allows coping with the structural changes in industrial plants without changing the existing structure of IWSNs. The feature adds flexibility to the network, however, it is also a challenge to establish a good communication link between source and destination without overloading a particular path and ensuring timely delivery of the information to the control unit.

The listed challenges and limitations encourage extensive research in IWSNs. In order to overcome these challenges and to achieve the design objectives and goals in IWSNs, prominent contributions have been made. Section VI to Section XI discuss some of such contributions and important research developments in IWSNs.

\section{Potential STANDARDS FOR IWSNS COMMUNICATION}

This section discusses different standards potentially suitable for IWSN. The discussion is divided in three sub-sections. Section VI-A discusses the less relevant wireless standards which could be and have been used for limited applications in WSNs. Section VI-B discusses more relevant WSN standards including IEEE802.15.4 and IEEE802.15.4e which discuss Physical and MAC specifications. Section VI-C focuses on the industrial protocols and standards which defined upper layers of the protocol stack using IEEE802.15.4 or IEEE802.15.4e as a baseline.

Wireless networks, have gained much popularity in this decade with many notable improvements in wireless industrial solutions. Significant reduction in individual mote price, improvement in processing and communication capabilities, development of protocols to facilitate communication and overcome interference are some of the improvements one can witness. All these improvements, especially the development of industrial wireless communication standards and protocols, assisted in wide scale implementation of IWSNs in industry. A detailed review of the wireless technologies, standards and industrial protocols for IWSNs are listed as follow.

The wireless technologies that fall in the domain of WPAN are standardized by 802.15 , a working group of IEEE. There are ten different groups working in 802.15, where each address certain aspects in standardization of WPAN.

1) IEEE 802.15.1 covers Bluetooth technology which is discussed in detail in Section VI-A(1).

2) IEEE802.15.2 addresses the coexistence issue in multiple devices operating in ISM band. A related discussion is presented in Section XII-F.

3) IEEE802.15.4 addresses the low datarate WPAN and plays a vital role in defining Physical and MAC layer specifications for low rate and low power networks including low-rate WPAN standard for industrial automation and process control. Further details can be found in Section VI-B.

4) IEEE802.15.5 addresses the issues of interoperability and scalability of wireless mesh networks in both low and high rate WPAN. A more detailed discussion regarding interoperability in low-rate WPAN can be found in Section XII.

Apart from these, other WPAN group focuses are listed as follows.

5) IEEE802.15.3 covers high-rate WPAN (suitability issues in industries due to high power requirements and unnecessarily high data rates compared to low data generated by sensors)

6) IEEE802.15.6 covers Body Area Networks (short range, unsuitable for industries)

7) IEEE802.15.7 covers Visible Light Communication (VLC) (Lack of existence of infrastructure and suitability to broader range of applications in industries)

8) IEEEP802.15.8 addresses peer to peer and infrastructure less communication (Fewer application that fall in this category in industrial automation and process control)

9) IEEE P802.15.9 addresses Key Management Protocol (KMP)

10) IEEE P802.15.10 addresses issues in routing in dynamically changing wireless networks (A less likely scenario due to the presence of static environments in industry)

The focuses of 802.15 groups listed in (5)-(10) are out of the scope of this paper. However, some details regarding possible developments regarding IWSNs can be found in Section IV$\mathrm{D}(5)$, discussing security and local key management, Section 
VI-A(3) discussing UWB as high-rate WPAN and Section XIIE covering potential of VLC in IWSN.

Furthermore, some other suitable standards apart from low-rate WPAN used in IWSNs under special circumstances including Bluetooth, Wi-Fi and Ultra-Wide Band (UWB), are also discussed. Whereas, more concerned area in WPAN standards, low-rate WPAN i.e. IEEE802.15.4 and IEEE802.15.4e are discussed in greater detail.

\section{A. Selected Wireless Technologies and Standards}

Many wireless technologies were considered to fulfil the needs of industrial applications. In all the technologies, tested for industrial wireless solutions, the aim was to benefit from the free unlicensed ISM band dedicated for industrial, scientific and medical ISM purposes. Wi-Fi, Ultra-Wide Band (UWB), and Bluetooth are three main technologies other than the IEEE802.15.4 based variants with potential to handle the industrial applications while utilizing the dedicated band. A brief discussion on benefits and shortcomings of these three technologies are listed as follows, while IEEE802.15.4, IEEE802.15.4e and industrial adaptation of these WPAN standards are discussed in detail in Section VI-B and Section VI-C respectively.

1) Bluetooth: Based on IEEE 802.15.1 standard, Bluetooth offers energy efficiency apart from low cost modules. In Bluetooth, seventy-nine channels are available with each offering a bandwidth of $1 \mathrm{MHz}$ to support high data rates [130]. Bluetooth devices use universal short-range radio link with frequency hopping spread spectrum (FHSS). FHSS ensures the security with the facility to access currently unoccupied channels. The protocol offers two connectivity topologies, piconet and scatternet [131]. Every piconet is formed by a Bluetooth device working as a master. The master-slave connection is established in a piconet where one master device can exist per piconet with one or more slave devices. A slave device can only establish a point to point link with the master, and can be put in the standby mode to improve energy efficiency. In a piconet, a master clock synchronization is also established. Multiple overlapping piconets form a scatternet where one device can be part of multiple piconets. However, a device can work as a master in only one piconet. Bluetooth devices offer a communication range of up to $100 \mathrm{~m}$, however, mostly the preferable communication range is up to $10 \mathrm{~m}$ [131].

Use of Bluetooth offers improved security, cost efficiency and reduced energy consumption, however, the intrinsic properties of Bluetooth limit the maximum number of connected nodes in a network. In earlier versions, only eight nodes could connect to an interface, hence affecting the suitability of Bluetooth in realistic industrial networks. With the introduction of Bluetooth 4.0, some of the constraints are relaxed. However, the master-slave interconnection in Bluetooth lacks flexibility and increases the protocol complexity. Furthermore, Bluetooth does not offer any support for mesh networking and fails to provide suitable mechanism for multi-hop communication, a core aspect of majority of the industrial wireless networks. Apart from these scatternet based multi-hop networks are inefficient and unsuitable for dense industrial networks [131],
[133], [134]. The lack of flexibility, support for limited nodes, master-slave link establishment, increased complexity and lack of multi-hop communication support are some of the issues, which affect the suitability of Bluetooth based solutions for fast paced and dynamic industrial networks [132], [131].

2) Wi-Fi: Based on IEEE 802.11standard, with possible variations of $802.11(\mathrm{a} / \mathrm{b} / \mathrm{e} / \mathrm{g} / \mathrm{n} / \mathrm{p} / \mathrm{ac} \ldots$ ay) [125], [126], [127], [128], [129], Wi-Fi offers a high data-rate using the frequency band of $2.401 \mathrm{GHz}$ to $2.473 \mathrm{GHz}$. Use of Wi-Fi allows large number of nodes, which improves the possibility for scalable networks. The network is formed using a centralized device to offer high data rates over short distances [132]. Several components in IEEE802.11 architecture interact to provide support for station mobility. A primitive cell consisting on mobile or fixed stations, is formed using Wi-Fi technology, referred as Basic Service Set (BSS) based on which IEEE802.11 employs independent basic service set (IBSS) and extended service set (ESS) network configurations [131]. IEEE 802.11 allows formation of ad-hoc networks where the stations can communicate without any Access Point (AP). An extended form of network can also be achieved using multiple BSS where the interconnection is established using Wireless Distribution System (WDS) [131]. However, WDS also has some disadvantages including throughput cut down for each WDS repeating hop, and elimination of rotated encryption key support. Furthermore, Wi-Fi modules are relatively expensive and consume more energy as compared to Zigbee and Bluetooth, which affect the suitability of Wi-Fi in battery operated networks [131]. With high power consumption, the lifetime of the IEEE802.11 networks is severely compromised, increasing maintenance and replacement costs. The short lifetime expectancy of IEEE 802.11 assisted networks also incurs unscheduled off times in the regular operation of industrial processes. Apart from these, high-speed data communication is not always desired, especially when the information is more vulnerable to interception due to high power transmission. Another issue that was noted in Wi-Fi networks was high multipath interference due to reflection of signals from the walls and other obstacles in indoor industrial environments [132]. Furthermore, increasing the number of devices in a single Wi-Fi connection, also affects the signal strength of the individual devices [132].

3) UWB: In UWB the information is communicated using very short pulses emitted in periodic sequence using radio frequency. Due to the use of impulses, UWB signal can be defined as an instantaneous spectral occupancy signal [135]. UWB has wide band of $500 \mathrm{MHz}$ with achievable data rates of $110 \mathrm{Mbps}$ [131]. Due to high achievable data rates, the UWB is termed as high-rate WPAN, also referred as IEEE802.15.3. UWB can be used in short-range applications and precise localization [133]. Due to the short range communication capabilities, UWB is used in indoor applications with high data rate requirements. High data rate in UWB) is suitable to assist multiple video and multimedia streams for indoor applications. Apart from this, UWB can fit in to short range cable replacement such as a wireless alternate for USB 2.0 and IEEE1394 [131].

The standardization and further developments of UWB include 
TABLE V: PHYSICAL Layer Parameters of IEEE 802.15.4 Standard [23]

\begin{tabular}{|c|c|c|c|c|c|c|}
\hline \multirow[t]{2}{*}{ PHY (MHz) } & \multirow{2}{*}{$\begin{array}{c}\text { Frequency Band } \\
(\mathrm{MHz})\end{array}$} & \multicolumn{3}{|c|}{ Data Parameters } & \multicolumn{2}{|c|}{ Spreading Parameters } \\
\hline & & $\begin{array}{l}\text { Symbol Rate } \\
\text { (kSymbols/s) }\end{array}$ & Symbols & $\begin{array}{c}\text { Bit Rate } \\
(\mathbf{k b} / \mathbf{s})\end{array}$ & Modulation & $\begin{array}{c}\text { Chip rate } \\
\text { kChip/s }\end{array}$ \\
\hline 780 & $779-787$ & 62.5 & 16-ary orthogonal & 250 & O-QPSK & 1000 \\
\hline 780 & $779-787$ & 62.5 & 16 -ary orthogonal & 250 & MPSK & 1000 \\
\hline $868 / 915$ & $\begin{array}{c}868-868.6 \\
902-928\end{array}$ & $\begin{array}{l}20 \\
40\end{array}$ & $\begin{array}{l}\text { Binary } \\
\text { Binary }\end{array}$ & $\begin{array}{l}20 \\
40\end{array}$ & $\begin{array}{l}\text { BPSK } \\
\text { BPSK }\end{array}$ & $\begin{array}{l}300 \\
600\end{array}$ \\
\hline 868/915 (optional) & $\begin{array}{c}868-868.6 \\
902-928\end{array}$ & $\begin{array}{c}12.5 \\
50\end{array}$ & $\begin{array}{l}\text { 20-bit PSSS } \\
\text { 5-bit PSSS }\end{array}$ & $\begin{array}{l}250 \\
250\end{array}$ & $\begin{array}{l}\text { ASK } \\
\text { ASK }\end{array}$ & $\begin{array}{c}400 \\
1600\end{array}$ \\
\hline 868/915 (optional) & $\begin{array}{c}868-868.6 \\
902-928\end{array}$ & $\begin{array}{c}25 \\
62.5\end{array}$ & $\begin{array}{l}16 \text {-ary orthogonal } \\
16 \text {-ary orthogonal }\end{array}$ & $\begin{array}{l}100 \\
250\end{array}$ & $\begin{array}{l}\text { O-QPSK } \\
\text { O-QPSK }\end{array}$ & $\begin{array}{c}400 \\
1000\end{array}$ \\
\hline 950 & $950-956$ & 100 & Binary & 100 & GFSK & \\
\hline 950 & $950-956$ & 20 & Binary & 20 & BPSK & 300 \\
\hline 2450 DSSS & $2400-2483.5$ & 62.5 & 16-ary orthogonal & 250 & O-QPSK & 2000 \\
\hline
\end{tabular}

IEEE P802.15.3a, IEEE 802.15.3b, IEEE 802.15.3c along with ongoing developments for amendment $3 \mathrm{~d}$ for IEEE 802.5.3. IEEE P802.15.3a operates in the frequency band of $3.1 \mathrm{GHz}$ to $10.6 \mathrm{GHz}$ with fractional bandwidth of $20 \%$ with the transmission range of $5 \mathrm{~m}$ and dynamic power range of 80 $\mathrm{dB}$ [136]. The improvements are targeted at imaging and multimedia communications. IEEE802.15.3b improves 2003 standard by adding interoperability to MAC along with some other features like MAC layer management entity, logical link control and added contention periods in frame. IEEE802.15.3c targeted millimetre wave based amendments in the Physical layer operating at a frequency of $57-64 \mathrm{GHz}$ with a communication range of $10 \mathrm{~m}$ [137].

Majority of the improvements introduced in the UWB target high-speed short-range communication which fail to align with the requirements of industrial applications. More suitable standards like IEEE 802.15.3 and IEEE 802.15.3b can at best serve as a supporting technology for IWSNs. Thus leaving UWB for industrial applications where the distance is relatively small with a need of extremely high data rate requirements. Furthermore, a relatively higher power requirement and ability to connect to eight motes at most, limit the scope of use of UWB for wide industrial market. High peak energy pulses also makes data more vulnerable to security attacks [135].

To give a quantitative comparison of power consumption of UWB and Wi-Fi, in comparison to tradition WSN, the power consumption of CC2430 (Zigbee), CX53111 (Wi-Fi) and XS110 (UWB) are presented in Fig. 7. The power consumption for transmission and reception of these modules is same due to the longer duration of active listening period compared to shorter transmission period [131].

\section{B. IEEE WPAN for WSNS/IWSNs}

IEEE WPAN standards offer a baseline for different working groups, covering details of the Physical and MAC layer. Based on the specifications of IEEE 802.15.4 [21] and 802.15.4e [20] ZigBee Alliance, ISA100 wireless compliance institute, HART communication foundation and other groups have defined protocols with upper layer specifications like ZigBee,

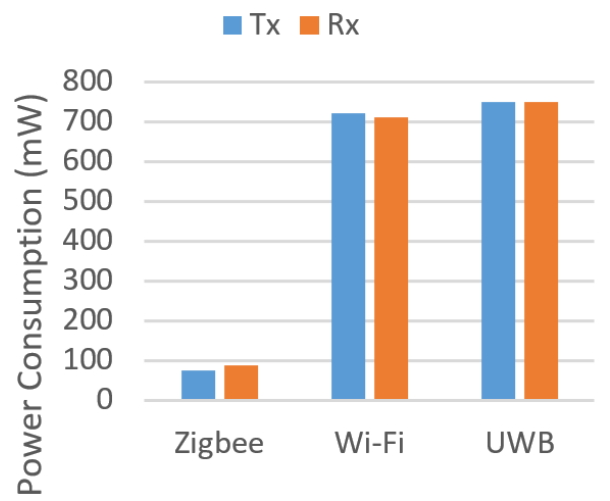

Fig. 7: Power consumption for Wi-Fi and UWB in comparison of Zigbee [131]

WirelessHART, ISA100.11a, 6LoWPAN and MiWi [27], [28], [29], [138].

1) IEEE 802.15.4: The standard offers specification of the Physical and MAC layer for low power, low cost, low speed and energy efficient communication within the nearby devices. The technology targets long life self-configurable network$\mathrm{s}$ with the ability of autonomous operation. The standard describes Physical and MAC layer architecture, functional overview, frame formats, management services, security operations, modulation schemes, transmission power, RF requirements and quality metrics.

Physical layer specifications provide frequency requirements, RF details, modulation schemes, spreading parameters, transmission power, channel details and assignment of UWB channels. It also specifies the recommended receiver sensitivity and link quality measure along with the channel state assessment. Details of some of the Physical layer parameters are listed in Table V.

The MAC layer handles the access to the physical channel including generation of beacons, synchronization mechanism to the generated beacons, motes association and disassociation to PAN. It also manages the assurance of contention free medium access by implementing CSMA/CA mechanism, support 
for device security, handling guaranteed time slot mechanism and reliable link assurance between the MAC entities [23]. In addition, the MAC layer controls the operating conditions of the motes by nominating them either as full-function device or reduced-function device. Full-function device can switch between a coordinator (a node which controls and coordinates a network) and a sensing device (a node which sense data and relay information in the network) whereas the reducedfunction device only works as a simple sensing device. The MAC layer also defines the layout of the superframe with the details of inter-frame separation, Contention Access Period (CAP) and contention Free Period (CFP).

2) IEEE 802.15.4e: The amendments in the existing WPAN standard IEEE 802.15.4 were focused on enhancing the suitability of existing standard for critical industrial applications of IWSNs. Thus, IEEE 802.15.4e mainly targets the real-time and reliability constraints in IWSNs. Some notable changes were introduced in the existing standard including the use of TDMA based channel access in IEEE 802.15.4e that replaced the CSMA/CA based access technique. This change offers guaranteed access to the channel to improve reliability. The changes introduced in new standard mainly targeted MAC layer with the inclusion of synchronization beacons for synchronization in new TDMA based access scheme. For retransmissions, shared slots are used which follow the CSMA/CA based access scheme with exponential back off (same mechanism is used in IEEE 802.15.4 during regular channel access schemes where the transmitter waits for random slots of time before retransmission, if the channel is busy). The modified standard also includes the structural amendments in the security header and control field.

\section{Selected Industrial Standards for WSNs/IWSNs}

Based on IEEE 802.15.4 and IEEE 802.15.4e many industrial protocols were formed to address the delicate nature and versatility of the industrial applications. Some of these protocols are listed as under. The listed protocols use Physical and MAC layer specifications of either IEEE 802.15.4 or IEEE 802.15.4e and extend their own upper layers model.

1) WirelessHART: WirelessHART is the technology solution in IWSNs, based on HART communication protocol developed by HART Communication Foundation [82]. With the built-in support for multiple IWSN topologies, WirelessHART offers solutions for monitoring, automation and process control for industrial applications. WirelessHART is widely accepted for industrial automation and process control with over 30 million HART devices installed worldwide [26]. The protocol is based on IEEE 802.15.4 standard with Direct Sequence Spread Spectrum (DSSS) and TDMA synchronized channel access mechanism. The protocol offers high reliability by incorporating the suggested modifications in IEEE 802.15.4e [139] along with channel hopping for enhanced security. It also supports the addressing of up to $2^{16}$ devices.

Link layer addressing is sufficiently large, enabling around 65000 devices within a single network but the network size is limited by the power consumption and latency issues. Nevertheless the WirelessHART also lacks in interoperability and fails compatibility to IP based devices and internet.
2) ZigBee: ZigBee, a protocol based on the IEEE 802.15.4 and developed by ZigBee Alliance offers a modest data rate of $250 \mathrm{kbps}$ [131]. It has sixteen channels each with a bandwidth of $2 \mathrm{MHz}$ and an ability to connect 65000 devices at once. These specifications allow the formation of mesh networks with single and multi-hop communication. ZigBee mainly focuses on low cost and low power solution for extended lifetime and improved deployment and maintenance cost.

It is one of the most widely used standards with over 70 million ZigBee devices installed worldwide [28]. Based on the IEEE 802.15.4, ZigBee provides upper layer specifications for a wide variety of applications. Currently, Zigbee Alliance, a non-profit open group offers three solutions referred as ZigBee PRO, ZigBee RF4CE and ZigBee IP. Apart from a relatively wider variety of solutions, ZigBee nodes are capable of connecting in mesh, star and tree topologies which further enhance the scope of its applications in industrial environment. Apart from this, a special feature in ZigBee PRO (ZigBee Green) allows battery less nodes to integrate with the networks, thus providing flexibility of greener technology.

ZigBee, on the other hand, uses CSMA/CA scheme for channel access which reduces its scope for time constraint and reliability critical industrial applications. Furthermore the exponential back-off mechanism triggered in case of channel unavailability introduces unwanted delay, which is not appreciated in time constraint industrial applications. Some of the overlapping channels in ZigBee and $\mathrm{Wi}-\mathrm{Fi}$ also introduce unwanted interference in the presence of Wi-Fi.

3) ISA100.11a: ISA100.11a is a wireless network solution by ISA100 Wireless Compliance Institute. ISA100.11a targets monitoring, automation and process control applications in industrial setup. A notable architectural resemblance is found in ISA100.11a and WirelessHART. For instance, the use of $2.4 \mathrm{GHz}$ operational frequency, implementation of TDMA based synchronized access and channel hopping functions in upper Data Link sub-layer are some of the many similarities in these two protocols. However, Network and Transport layer of ISA100.11a are derived from 6LoWPAN [139], which allows the use of IPv6 addressing in this standard.

The MAC sub-layer uses CSMA/CA mechanism for the channel access. However, retransmissions can benefit from frequency, time and spatial diversity. An optional implementation of IEEE 802.15.4, CSMA/CA based exponential back-off mechanism is also available. It also allows implementation of TDMA based channel access and channel hopping with ARQ interference suppression mechanism.

4) 6LoWPAN: 6LoWPAN is a IPV6 based low power wireless personal area network [140], [141]. 6LoWPAN offers the benefit of interfacing directly with other IP devices or existing IP networks. It also inherits the security, architecture, network management and transport layer protocols from the existing structure. The use of IPV6 enables the 6LoWPAN devices to readily embed in the existing wired industrial Ethernet setup. To ensure low power operation, the superframe is divided into active and inactive regions where the coordinator can go into low power or sleep mode to conserve energy. Low power listening mode is also included to further improve the energy efficiency. To provide 
TABLE VI: Selected Industrial Protocols and Standards [20], [23], [27], [28], [29], [138], [142]

\begin{tabular}{|c|c|c|c|c|c|c|c|c|c|c|}
\hline $\begin{array}{c}\text { Industrial } \\
\text { Standard } \\
\text { and Protocols }\end{array}$ & $\begin{array}{c}\text { Research group } \\
\text { / Institute / } \\
\text { Working Alliance }\end{array}$ & $\begin{array}{c}\text { Multi- } \\
\text { Topologies } \\
\text { Support }\end{array}$ & $\begin{array}{c}\text { Estimate } \\
\text { of } \\
\text { Devices }\end{array}$ & $\begin{array}{c}\text { Access } \\
\text { Scheme }\end{array}$ & $\begin{array}{c}\text { Channel } \\
\text { Access }\end{array}$ & $\begin{array}{c}\text { Network } \\
\text { Size }\end{array}$ & $\begin{array}{c}\text { Base } \\
\text { Standard }\end{array}$ & $\begin{array}{c}\text { Interoper- } \\
\text { ability } \\
\text { (IP \& } \\
\text { Internet) }\end{array}$ & $\begin{array}{c}\text { Green } \\
\text { Motes } \\
\text { Support }\end{array}$ & $\begin{array}{l}\text { Data } \\
\text { Rate } \\
\text { kbps }\end{array}$ \\
\hline $\begin{array}{c}\text { WirelessHART } \\
{[82],[138]}\end{array}$ & $\begin{array}{c}\text { HART } \\
\text { Communication } \\
\text { Foundation }\end{array}$ & Available & $\begin{array}{c}30 \\
\text { Million }\end{array}$ & $\begin{array}{c}\text { Direct } \\
\text { Sequence } \\
\text { Spread } \\
\text { Spectrum } \\
\text { (DSSS) }\end{array}$ & TDMA & $2^{16}$ & IEEE 802.15 .4 & No & No & 250 \\
\hline $\begin{array}{c}\text { Zigbee } \\
\text { [21], [143], [144] }\end{array}$ & $\begin{array}{c}\text { Zigbee } \\
\text { Alliance }\end{array}$ & Available & $\begin{array}{c}70 \\
\text { Million }\end{array}$ & DSSS & CSMA/CA & $2^{16}$ & IEEE 802.15 .4 & $\begin{array}{c}\text { Yes } \\
\text { (Zigbee } \\
\text { IP) }\end{array}$ & $\begin{array}{c}\text { Yes } \\
\text { (Zigbee } \\
\text { Pro) }\end{array}$ & 250 \\
\hline $\begin{array}{c}\text { ISA100.11a } \\
{[20],[82],[139]}\end{array}$ & $\begin{array}{c}\text { ISA100 } \\
\text { Wireless } \\
\text { Compliance } \\
\text { Institute }\end{array}$ & $\begin{array}{c}\text { Mesh } \\
\text { Routing }\end{array}$ & - & $\begin{array}{c}\text { DSSS } \\
\text { Channel } \\
\text { Hopping }\end{array}$ & $\begin{array}{c}\text { TDMA } \\
\text { (Transport } \\
\text { Layer), } \\
\text { CSMA } \\
\text { (MAC) }\end{array}$ & $\begin{array}{c}\text { IPV6 } \\
\text { Addressing }\end{array}$ & IEEE 802.15 .4 & $\begin{array}{c}\text { Yes } \\
\text { (IPV6) }\end{array}$ & No & 250 \\
\hline $\begin{array}{l}\text { 6LoWPAN } \\
\text { [27], [141] }\end{array}$ & $\begin{array}{c}\text { Internet } \\
\text { Engineering } \\
\text { Task Force }\end{array}$ & Available & - & - & CSMA/CA & $\begin{array}{c}\text { IPV6 } \\
\text { Addressing }\end{array}$ & IEEE 802.15 .4 & $\begin{array}{c}\text { Yes (IPV6), } \\
802.15 .4 \\
\text { Compliance }\end{array}$ & No & $20-250$ \\
\hline
\end{tabular}

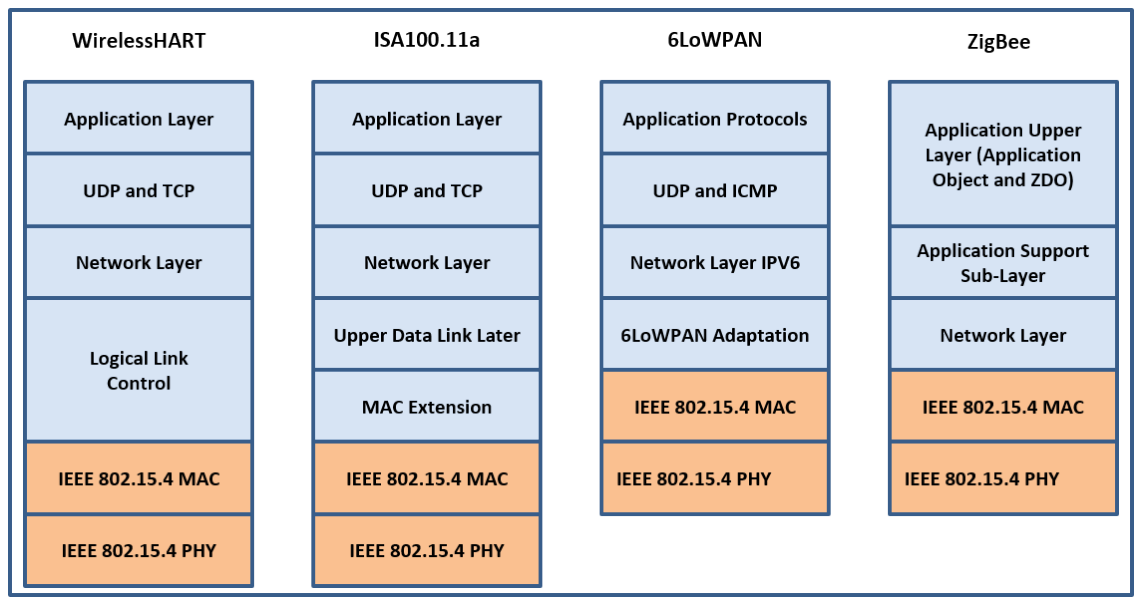

Fig. 8: Protocol Stack Structure for selected Industrial Standards [82], [139], [141]

security from external attacks, the protocol incorporates 128-bit AES. To extend the scope of 6LoWPAN, the ability to interact with MAC devices is included which enables the 6LoWPAN devices to integrate with other IEEE802.15.4 based devices.

On the other hand, in 6LoWPAN, the channel access and reliability are a bit compromised with the use of CSMA/CA based channel access. To initiate transmission, the devices have to compete for the channel access using CSMA/CA based mechanism which adds uncertainty. Moreover, the protocol support low data rates ranging from 20 to $250 \mathrm{kbps}$. A brief overview of the selected industrial protocols is presented in Table VI whereas the protocol stack of the above mentioned industrial protocols and responsibilities of different layers in the protocol stack are presented in Fig. 8 and Table VII respectively.

The technological developments in the past few years, whether it involves hardware platforms or standardization of access schemes, leaves a significant impact on improving credibility of IWSNs. A review of the milestones achieved in last two decades is presented in Fig. 9. It pinpoints the main contributions and milestones achieved in hardware platform design, standards and industrial protocols. Although, a more detailed description of the milestones represented in taxonomy in Fig. 9 can be found in Section VI, VII and XII, yet the taxonomy presents broader perspective of significant events in the past. The taxonomy also gives a fair insight in the future market value of IWSNs and potential of future technologies in industrial applications.

\section{IWSN PlatForms, Field-tRials, Simulators AND SERVICE PROVIDERS}

\section{A. Industrial Motes and Available Radios}

Wireless sensor motes used for industrial applications are equipped with a processor, memory, sensor board, radio, 


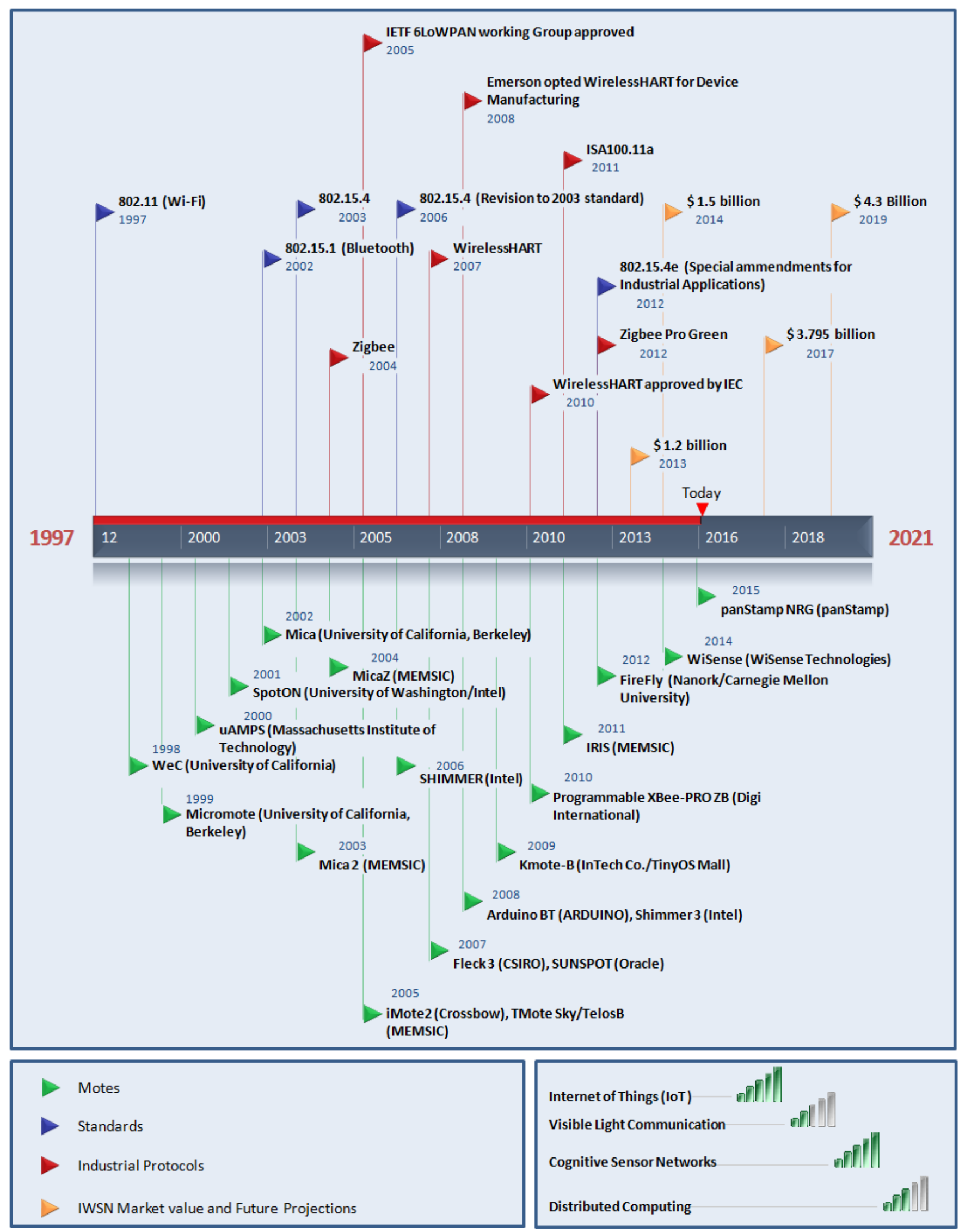

Fig. 9: Taxonomy of Wireless Motes, Standards, Industrial Protocols, IWSNs Market value \& Projections and Future Industrial Technologies [20], [21], [22], [23], [26], [27], [28], [29], [129], [130], [142], [145], [146], [147], [148] 
TABLE VII: Salient Features of Protocol Stack of selected Industrial Standards

\begin{tabular}{|c|c|c|c|c|}
\hline \multirow{2}{*}{ Layers } & \multicolumn{4}{|c|}{ Functionality } \\
\hline & WirelessHART & ISA100.11a & Zigbee & 6LoWPAN \\
\hline Application Layer & - HART command [51] & $\begin{array}{l}\text { - Publishing / Subscribe, } \\
\text { Client/Server, bulk, Alert } \\
\text { event notification } \\
\text { - Wired fieldbus protocol } \\
\text { interoperability by Object } \\
\text { mapping or protocol } \\
\text { tunneling [51], [82] }\end{array}$ & $\begin{array}{l}\text { - ZigBee Application Layer } \\
\text { [51] }\end{array}$ & $\begin{array}{l}\text { - HTTP } \\
\text { - Constrained application Pro- } \\
\text { tocol (COAP) } \\
\text { - Message queue telemetry } \\
\text { transport (MQTT) } \\
\text { - Websocket [27], [355] }\end{array}$ \\
\hline Transport Layer & $\begin{array}{l}\text { - Connection orient and } \\
\text { connection less communi- } \\
\text { cation } \\
-\quad \text { Transport layer ack- } \\
\text { nowledgements } \\
-\quad \text { Message-based Priority } \\
{[51],[82]}\end{array}$ & 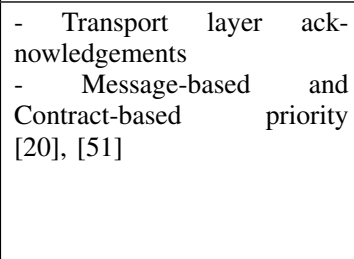 & - & $\begin{array}{l}\text { - UDP preferred due to low } \\
\text { overhead and energy load } \\
\text { over TCP } \\
\text { - TCP } \rightarrow \text { Transport Layer Se- } \\
\text { curity (TLS) } \\
\text { - UDP } \rightarrow \text { Datagram Trans- } \\
\text { port layer security (DTLS) } \\
{[355]}\end{array}$ \\
\hline Network Layer & $\begin{array}{l}\text { - Extended HART Address } \\
\text { [51] }\end{array}$ & $\begin{array}{l}\text { - IPv6 addressing (6LoW- } \\
\text { PAN) [51] }\end{array}$ & $\begin{array}{l}\text { - Zigbee Routing Algorithms } \\
\text { - Route Aggregation } \\
\text { - Tree routing } \\
\text { (AODV) [21], [51] }\end{array}$ & $\begin{array}{l}\text { - IPv6 routing protocol for } \\
\text { low-power and lossy network- } \\
\text { s (RPL) [355] }\end{array}$ \\
\hline Data Link Layer & $\begin{array}{l}\text { - TSMP features } \\
\text { - Superframe Optimization } \\
\text { - No frequency reuse for ded- } \\
\text { icated links [51], [138] }\end{array}$ & $\begin{array}{l}\text { - TSMP features } \\
\text { - CSMA/CA } \\
\text { - Channel Hopping (SH, FH, } \\
\text { Hybrid) } \\
\text { - AFH and BlackListing } \\
\text { - Superframe Optimization } \\
\text { [51] }\end{array}$ & $\begin{array}{l}\text { - CSMA/CA } \\
\text { - Beacon Sync. } \\
\text { - Frequency Agility [51], } \\
{[143]}\end{array}$ & $\begin{array}{l}\text { - Adaptation of IPv6 to IEEE } \\
802.15 .4 \\
\text { - CSMA/CA } \\
\text { - Beacon Sync [27], [355] }\end{array}$ \\
\hline Physical Layer & $\begin{array}{l}\text { - IEEE } 802.15 .4,2.4 \mathrm{GHz}, \\
868 \mathrm{MHz}, 915 \mathrm{MHz} \\
\text { - DSSS } \\
\text { - Data rate } 250 \mathrm{~Kb} / \mathrm{s} \text { [23], [82] }\end{array}$ & $\begin{array}{l}\text { - IEEE } 802.15 .4,2.4 \mathrm{GHz} \text {, } \\
868 \mathrm{MHz}, 915 \mathrm{MHz} \\
\text { - DSSS } \\
\text { - Data rate } 250 \mathrm{~Kb} / \mathrm{s}[21]\end{array}$ & $\begin{array}{l}\text { - IEEE } 802.15 .4,2.4 \mathrm{GHz}, \\
868 \mathrm{MHz}, 915 \mathrm{MHz} \\
\text { - DSSS } \\
\text { - Data rate } 250 \mathrm{~Kb} / \mathrm{s}[21],[23]\end{array}$ & $\begin{array}{l}\text { - IEEE } 802.15 .4,2.4 \mathrm{GHz}, \\
868 \mathrm{MHz}, 915 \mathrm{MHz} \\
\text { - DSSS } \\
\text { - Data rate } 250 \mathrm{~Kb} / \mathrm{s} \text { [21], [27] }\end{array}$ \\
\hline
\end{tabular}

battery and probable extensions for energy harvesting and protective packaging. On the software end the software platfor$\mathrm{m}$, developer tool and simulators provide substantial support. All these factors contribute equally and serve as a decisive factor in adoption of a particular mote for certain process control application. For instance, a process, which requires lot of different sensors and high sampling rates, may demand a mote with more flexible external sensor attachment options and a relatively wide band communication standard. Some other applications may demand connectivity to a relatively larger network, which may disqualify certain Bluetooth, based wireless modules [149].

Industrial motes are application specific which allow a low power, efficient and delay sensitive solution. Furthermore, most of the industrial solutions are reinvented with modular based solutions, where the radio requirements, processing needs, temperature tolerance, moisture resistivity, memory requirements, interoperability and channel access schemes are specifically modelled to offer a low cost effective solution, fully optimized for the application at hand. As an example, if a particular industrial application requires an 8-bit, $4 \mathrm{MHz}$ processor, capable of executing 1 Million Instructions Per Second (MIPs) to sense, sample, synchronize and communicate information, there is no point of adding a 32 bit architecture working at $48 \mathrm{MHz}$, and processing $30 \mathrm{MIPS}$ : one, it will add additional cost to the solution (more critical for larger number of deployments), two, it will consume more power, being running on higher clocks, three, more heat is produced and affiliated heat dissipation issues arise.

For industrial automation and process control many commercial solutions are available along with some research initiatives to fill the gaps. All these works focus on more flexible solution design, targeting mainstream industrial applications. Some of the motes and radio modules designed for industrial automation are listed as follows. Please note that each of discussed motes, radios and System on Chip (SoC) are labelled with M (Mote), R (Radio) or SoC.

1) WirelessHART compliant solutions for industrial automation:

- LTC5800 (SoC): LTC5800 is designed by dust networks to achieve a high reliability of the order up to $99.999 \%$ to minimize the risk in the industrial applications. The LTC5800 based motes are wirelessHART compatible and offers time synchronized network-wide scheduling, per transmission frequency hopping, network wide reliability and low power operation [150]. LTC5800 belongs to Eterna family of SoC, which provides a scalable, reliable, energy efficient and robust networking solution.

- LTP5900-WHM (M): LTP5900-WHM features ARM Cortex-M3 32-bit microprocessor which runs WirelessHART, enabling it to form mesh networks with selfhealing ability. The physical and MAC layer are adopted from IEEE802.15.4 and IEEE802.15.4e respectively where encryption and authentication is also ensured [151]. It includes temperature and drift compensation for real-time network synchronization. Frequency hopping 
and reliability optimization is ensured. Apart from this, smart networking technology allows LTP5900 to form self-healing mesh networks.

The core communication in the WirelessHART based solutions listed above is very similar and only minor changes are introduced. All these solutions offer good reliability and scalable modular design however, do suffer from interoperability issues.

2) Zigbee and 6LoWPAN compliant solutions:

- Kinetis KW2xx (M): Panasonic Kinetis KW2xx series implements SMAC and Thread. Thread [152] being an IPv6 mesh networking protocol developed by industry leading technology companies, offers better interconnectivity, Over The Air Programming (OTAP) and support for multiple IDEs and Real Time Operating Systems (RTOS). Some of the Kinetis KW2xx includes ARM Cortex-M4, 32-bit microprocessors which also implements IEEE802.15.4 [153]. KW21Z, being on the low end uses Cortex-M0 to enable energy efficient operation. The application areas of Kinetis KW2xx in industries include climate control, safety access control and security [152].

- JN517x series (M): JN517x series by NXP supports Zigbee 3.0, which bridges the gap between the MAC devices and IoT by offering suitable interconnectivity. It is also compatible with Thread and IEEE802.15.4 [154].

- Ember EM35x (SoC): Silicon Labs Ember EM35x ARM SoCs are Integrated with Zigbee. The use of ARM Cortex-M3 based Zigbee SoCs are used which offer better energy efficiency and improved performance. AES 128 encryption is also implemented for improved security [155].

- OpenMote (M): OpenMote follows modular approach where CC2538, OpenBase and battery are connected together to offer extended performance [156]. Although openMote is more research oriented solution yet the use of CC2538 in OpenMote offers many of the shelf benefits and is found in many industrial applications as well. It is a SoC from Texas Instruments operating at $2.4 \mathrm{GHz}$ band. It is fully compatible with IEEE 802.15.4 and provides both 6LoWPAN and Zigbee implementations on the higher layers [157].

- Re-Mote (M): Re-Mote from Zolertia also uses CC2538 [157] and hence possesses similar features as the OpenMote. However, Re-Mote is particularly optimized for the IoT [158]. Apart from this, Re-mote also features CC1200 [159] for $868 / 915 \mathrm{MHz}$ band communications which is facilitated by multi-band antenna.

- Z1 (M): Z1 by Zolertia is designed to target both commercial and industrial applications. To offer full compliance to IEEE802.15.4 and 6LoWPAN, Z1 is equipped with CC2420 and MSP430 radios It also provides support for TinyOS and Contiki [160].

All these motes are suitable for small scale industrial mesh networking. With the increase in the number of nodes the operation is significantly compromised. Therefore, these motes are not suitable for critical processes especially if a large number of sensor motes need regular communications within

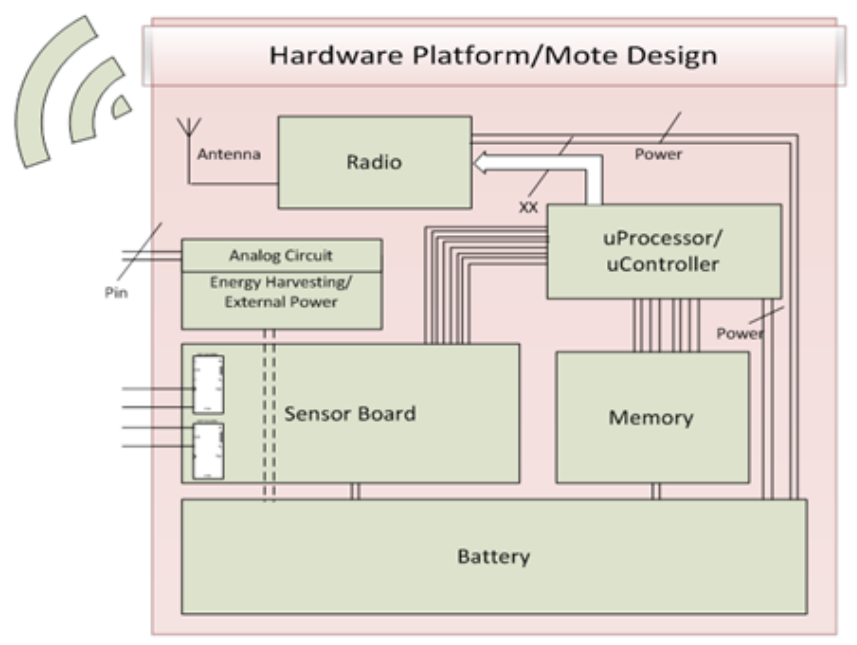

Fig. 10: IWSN Mote Hardware Architecture

a specified time.

3) XYR 6000 (ISA100.11a compliant (SoC)): XYR 6000 are developed by Honeywell as a replacement to seamlessly expensive wired links, especially for where using wired links are either too costly, time consuming, inaccessible or the instrumentation schemes are frequently changing. XYR 6000 implements IEEE802.15.4 Physical layer and IEEE802.15.4e MAC layer. These modules are integrated in Honeywell OneWireless network and are compliant with ISA100.11a. The gateway is capable to communicate with up to 100 nodes within a given time. The gateway and associated nodes also offer IEEE $802.11 \mathrm{~b} / \mathrm{g}$ interface for improved interoperability. XYR 6000 also offer end to end industry security, multispeed monitoring, reliability, improved performance, and ruggedness for industrial hazardous environments [161].

4) Wi-Fi/Bluetooth enabled motes:

- RN1810/RN1810E (SoC): RN1810 is created by Microchip and it incorporates an on-board TCP/IP networking stack, $2.4 \mathrm{GHz}$ transceiver, cryptographic accelerator, real-time clock, RF power amplifier and power management subsystem. The module is capable of providing data rates of up to $2 \mathrm{Mbps}$ with an extended range of 400 meters [162]. The SPI interface allows the module to integrate with Microchip PIC microcontroller family.

- muRata Com 6M (SoC): It is a TIs WL1271L chipset based module which provides Bluetooth 4.0, Bluetooth Low Energy (BLE) and IEEE802.11b/g/n connectivity. muRata Com6M integrates with TIs OMAP, DaVinci and Integra to offer extended features.

- Intel Mote (IMote) and IMote2 (M): The design of IMote is optimized to offer improved performance, bandwidth efficiency, low power operation and cost effectiveness. It includes an ARM7 core and IEEE802.15.1 (Bluetooth) compatible radio. To improve the connectionoriented Bluetooth nature, network formation and maintenance algorithms are optimized with the adoption of scatternet [163], [164].

These modules offer high data rates which enables them to offer high speed data links. While there are many traditional 
TABLE VIII: Technical Specifications of Selected Motes [167], [168], [169], [170], [171]

\begin{tabular}{|c|c|c|c|c|c|c|}
\hline Mote Platform & Micro-Processor & RAM & Flash & EEPROM & Bus & Clock \\
\hline TelosB/Tmote Sky & TI MSP430F1611 & $10 \mathrm{~K}$ & $48 \mathrm{~K}$ & $1 \mathrm{M}$ & $16-$ bit & $4-8 \mathrm{MHz}$ \\
\hline MicaZ/Mica2 & Atmel Atmega 128L & $4 \mathrm{~K}$ & $128 \mathrm{~K}$ & $512 \mathrm{~K}$ & 8 -bit & $8 \mathrm{MHz}$ \\
\hline FLECK 3B & Atmel ATmega 128L & $8 \mathrm{~K}$ & $128 \mathrm{~K}$ & - & 8 -bit & $8 \mathrm{MHz}$ \\
\hline IRIS & Atmel ATmega 128L & $8 \mathrm{~K}$ & $640 \mathrm{~K}$ & $4 \mathrm{~K}$ & 8 -bit & $8 \mathrm{MHz}$ \\
\hline Sun SPOT & Atmel AT91RM9200 & $512 \mathrm{~K}$ & $4 \mathrm{M}$ & none & $32-$ bit & $180 \mathrm{MHz}$ \\
\hline EZ-RF2480 & TI MSP430F22741 & $1 \mathrm{~K}$ & $32 \mathrm{~K}$ & none & $16-$ bit & $16 \mathrm{MHz}$ \\
\hline JN5139 & JN5139 & $96 \mathrm{~K}$ & $192 \mathrm{~K}$ & - & $32-$ bit & $16 \mathrm{MHz}$ \\
\hline RCM4510W ZB & Rabbit 4000 & $512 \mathrm{~K}$ & $512 \mathrm{~K}$ & - & 8 -bit & $29.49 \mathrm{MHz}$ \\
\hline PAPER & Atmel Atmega 128L & $132 \mathrm{~K}$ & $128 \mathrm{~K}$ & - & 8 -bit & $8 \mathrm{MHz}$ \\
\hline
\end{tabular}

TABLE IX: Radio Specifications of Selected Motes [172], [173], [174], [175]

\begin{tabular}{|c|c|c|c|c|c|c|}
\hline Mote Platform & Radio Module & Sleep & Idle/Rx & Tx(mA) & Frequency (MHz) & Data Rate (Kbps) \\
\hline Mica2 & TI CC1000 & $0.2 \mu \mathrm{A}$ & $<7.4 \mathrm{~mA}$ & 10.4 & 900 & 40 \\
\hline $\begin{array}{c}\text { TelosB/Tmote } \\
\text { SunSPOT/MicaZ/SHIMMER }\end{array}$ & TI CC2420 & $0.02-426 \mu \mathrm{A}$ & $18.8 \mathrm{~mA}$ & 17.4 & $2400-2483.5$ & 250 \\
\hline EZ-RF2480 & TI CC2480 & $0.3-190 \mu \mathrm{A}$ & $26.7 \mathrm{~mA}$ & 26.9 & 2400 & 250 \\
\hline IRIS & Atmel AT86RF230 & $20 \mathrm{nA}$ & $15.5 \mathrm{~mA}$ & $16.5(3 \mathrm{dBm})$ & $2405-2480$ & 250 \\
\hline RCM4510W & MaxStream XBee 2 & $<20 \mu \mathrm{A}$ & $150 \mathrm{~mA}$ & 150 & 2400 & 250 \\
\hline FLECK 3B & Nordic RF 905 & $33 \mu \mathrm{A}$ & $12.5 \mathrm{~mA}$ & 30 & $433 / 868 / 915$ & 50 \\
\hline JN5139 & Custom RF board & $2.6 \mu \mathrm{A}$ & $<37 \mathrm{~mA}$ & 37 & 2400 & 250 \\
\hline PAPER & MaxStream 9XCite & $100 \mu \mathrm{A}$ & $<77 \mathrm{~mA}$ & 77 & $902 / 928$ & 40 \\
\hline
\end{tabular}

applications of these modules, the common industrial applications where these modules can be seen, include, supervisory control, high speed data bridging, monitoring, vibration and chemical sensing.

5) Waspmote (M): While not particularly designed for only industrial industrial automation, waspmote has certain features which make the waspMote more appealing for the industrial environments. Use of Waspmote for smart water sensing and air quality monitoring are specifically the applications of interest [165], [166]. The demonstration of smart water sensor using Waspmote uses the peripheral sensors to measure water quality parameters, like dissolved ion content, oxygen levels, conductivity and $\mathrm{pH}$ [165]. New generation of Waspmotes launched by Libelium, provides open source platform for wireless sensor which now integrates 60 different sensor probes to facilitate the gas quality monitoring and evaluation of $\mathrm{NO}$, $\mathrm{CO} 2, \mathrm{CO}$ and other harmful gases levels in different industrial environments [166]. The modular approach used in Waspmote also offers a number of radio technologies including, WiFi, Zigbee, 802.15.4, Bluetooth, NFC and 3G, to choose from for extended applications and improved operability [166]. The new generation also supports OTAP for flexible and remote operations.

6) Some significant secondary research motes and WSN/IWSN platforms: Apart from the motes listed above, there is a wide variety of wireless motes available in the market or developed by the researchers for diverse applications. All these solutions offer suitable variety to meet a wide range of applications including home automation, research and development, smart cities, lighting control etc. Some of the prominent motes with technical specifications and radio details [16], [56] are listed in Table VIII and Table IX respectively. Whereas a generalized architecture of WSNs/IWSNs Hardware Platform is presented in Fig. 10. It is also worth noticing that the hardware attributes of WSNs and IWSNs are quite similar since the physical layer, radio specifications, processing, storage and hardware features are almost the same however, some of the application specific attributes of IWSN motes are listed as follows.

1) TDMA/guaranteed channel access/time synchronization/ packet scheduling/link heterogeneity

2) Priority based channel access for emergency and regulatory control

3) Data integrity check/ Encryption, symmetric key cryptography (AES-128, key management, frame protection)

4) QoS assurance/ Multi-hop network support/Fault tolerance

5) Explicit congestion notification

6) upstream/downstream reliability/ loss recovery

7) Implicit and explicit congestion notification

Apart from these, motes are designed to withstand harsh industrial environments where the operation under high temperature or pressure may be required.

\section{B. Field-trials}

IWSN is emerging as a cost efficient and effective networking solution for industrial automation. A number of cases have been presented where compliance with international standards is ensured using IWSNs. Some of such field-trials for industrial automation are presented as follows.

1) Polibol: Smart Factory solution in Zaragoza, Spain: Polibol is a manufacturing company which produces aluminium laminated plastics and printed coils for food products 
TABLE X: Companies Offering Services in IWSNs [176], [177], [178], [179], [180], [181], [182], [183], [184], [185]

\begin{tabular}{|c|c|c|l|}
\hline Sr. & Companies & Country & Scope/Services \\
\hline 1. & Emerson & US & Automotive, Beverage, Food, Metals, Oil and Gas, Packaging, Pulp and Paper, Solar, Wind \\
\hline 2. & GE Energy & US & $\begin{array}{l}\text { Critical Power Services, Drives Services, Emergency Services, Engineering Services, Hydro } \\
\text { Services, Inspection Services, Mechanical Services }\end{array}$ \\
\hline 3. & Honeywell & US & Energy, Safety, Security \\
\hline 4. & ABB & Switzerland & Control Products and Systems, Industries and Utilities, Measurement Products \\
\hline 5. & Endress+Hauser & Switzerland & $\begin{array}{l}\text { Energy Solutions, Analytical Solutions, Field Network Engineering, Plant Asset } \\
\text { Management }\end{array}$ \\
\hline 6. & Siemens & Germany & Automation, Building Technologies, Energy, Healthcare \\
\hline 7. & Yokogawa & Japan & $\begin{array}{l}\text { Chemical, Food and Beverage, Iron and Steel, LNG Supply Chain, Oil and Gas, Power, } \\
\text { Refining, Renewable Energy, Concentrated Solar Power, Wind Power }\end{array}$ \\
\hline 8. & Yamatake & Japan & Sensors, Process Controls, Harsh Environment \\
\hline 9. & Invensys & UK & $\begin{array}{l}\text { Production Optimization, Real-Time Performance Management, Industrial Manufacturing } \\
\text { Facilities Performance, Control Excellence, Industrial Power Management, Net Oil and Gas } \\
\text { Solution, Environment and Safety Excellence, Environmental Monitoring System }\end{array}$ \\
\hline 10. & Mitsubishi & Japan & Industrial solutions, Energy \\
\hline
\end{tabular}

and consumer industry. It operates several production lines that incur automation of critical processes including control of air temperature, gas monitoring and ensuring authorized gas concentration levels. Since, Polibol manufactures flexible food packaging, it must follow demanding health legislation and food hygiene [448]. To ensure the compliance with international standards and FDA regulations, a high level of quality control must be established throughout the production. Recently, Polibol has introduced Libelium's Waspmote and Meshlium based IWSN solution to ensure high level of automation in one of the production facilities in Zaragoza, Spain. Libelium's Waspmote based sensor network is used to monitor the air temperature in pipes and around the printing machines, and carbon dioxide concentration in working areas in realtime [449]. Whereas Meshlium serves as an IoT gateway that connects Libelium wireless network to Microsoft Azure cloud [450], [451]. Libelium's Waspmote and Meshlium based IWSN ensures real-time data communication. IWSN based real-time process control not only improves quality of the products but also reduces the maintenance costs.

2) Honeywell: Nucor Steel in Tuscaloosa, Alabama: Honeywell is one of the leading services provider in automation and process control industry. The use of IWSNs in the automation, monitoring and feedback control systems, has resulted in several economic benefits along with improved safety. Implementation of wireless transmitters to monitor the furnace temperature at Nucor Steel in Tuscaloosa (Nucor Corporation is largest steel manufacturer in US) is one such project which resulted in increasing production by 15 percent [452].

Primary objective was to improve the process operations by effectively establishing instantaneous temperature feedback from one of the furnace, previously not being monitored. The wireless solution was provided by Honeywell using the wireless nodes mounted on the cooling circuits protected by specially built cases to withstand temperature of up to $1000^{\circ}$ centigrade [453].

3) OneWireless EHM case study: Loch Rannoch: Loch Rannoch is a double-hull oil tanker, capable of transporting
130,000 tons. It is used for transporting oil from storage vessel to an oil processing terminal in the North Sea [453]. Due to the large size of Loch Rannoch, manual monitoring is not a viable option. Further, to this, the purpose of the monitoring system was to ensure safety of the employees, protection of the on-board assets, improvement in tankers capacity and proactive management of maintenance schedules. The listed objectives were achieved using OneWireless [454] based wireless network solution where a highly redundant network was formed to overcome the poor channel conditions on the tanker. The implementation of WSN in Loch Rannoch resulted in increased uptime, time efficiency and reduced cost [453].

4) Dust Networks, wafer manufacturing in California, USA: Semi-conductor wafer manufacturing units use delicate processes for the production of high quality wafers. On the same time, to maximize uptime, and overall yield of the processes, uninterrupted supply must be ensured.

At Linear technologies Silicon Valley fabrication unit, over one hundred and seventy-five gas cylinders are used in the wafer manufacturing process [455]. In this facility, an unplanned interruption in the gas supply can cause significant financial loss and an unplanned delay in the supply to the customers. Use of wired networks was not possible due to the presence of concrete walls and lack of AC sockets. Wi-Fi was also not a suitable option due to the channel distortion and high interference [455].

To ensure the uninterrupted operation of the wafer manufacturing plant, IWSN based automation and feedback control system is adopted. Linear Technology used a SmartMesh IP WSN to streamline manufacturing operations, monitoring gas cylinder levels to proactively schedule replenishment and ensure uninterrupted supply. SmartMesh IP embedded wireless mesh networks worked effectively with a reported data reliability of up to $99.99999 \%$ along with ultra-low power power requirements [455], [456]. 


\section{Network Simulators}

The researchers and industries widely use network simulators to evaluate the performance of developed scenarios with certain level of accuracy. Use of simulators in WSNs, cuts down the initial cost and give viable insight on performance of wireless ad-hoc networks before the actual deployment. Various network simulators are currently available. Some of the prominent network simulation and evaluation tools are discussed as follows.

1) NS2/NS3: Network simulator, $\mathrm{NS} 2$ is a discrete event simulator which offers OTcl script and $\mathrm{C}++$ based tool to evaluate performance of wired and wireless networks. NS2 offers suitable support to develop new and customizable libraries and include extensive features in the existing package [457]. The customizable nature of NS2 makes it suitable for network performance evaluation in WSNs. The NS2 framework allows definition of new packet headers and packet tracers for improved debugging. Third party extensions in NS2 can also be easily incorporated ns2miracle. Over the years various WSN standards and protocols have been implemented and evaluated using NS2 [459]. However, the use of OTcl causes large overhead, resulting from the OTcl interfacing with $\mathrm{C}++$ and OTcl interpreter. To improve the overall efficiency of NS2, and to overcome its limitations, NS3 was introduced, however, the lack of back compatibility in NS3 restricts the integration of the WSN based framework and libraries already developed for NS2 [460].

2) $\mathrm{OMNeT++}$ : OMNeT++ is a $\mathrm{C}++$ based framework used for developing network simulators. With the use of OMNeT simulation library, many network simulation packages have been developed [461]. The evaluation of WSN based network is supported by some of the extension packages developed using OMNeT++. Some examples of OMNeT based WSN supported extension packages include Mobility Framework (MF), Castalia [462] and MiXiM.

Castalia is widely used in the research community for simulation of general purpose WSN, Body Area Networks (BAN) and low power networks [463]. Use of Castalia in WSNs is limited to generally static networks where the mobileelement based network preferably use MF or MiXiM. Castalia provides support for wide range of platforms and allows to evaluate their performance under various circumstances. The performance of the network can be evaluated based on several parameters using interactive simulation.

MF and MiXiM , are simulators for wireless and mobile networks, developed using OMNeT++ simulation engine. Mixim itself was a merger of four simulator including MF, Mac Simulator, Positif Framework and ChSim. Many other models and projects have also been integrated in MiXiM. As the MiXiM inherited all the features of MF and other mobile networking frameworks, therefore, it provides substantial support in formulation and evaluation of various wireless and mobile network scenarios [464].

3) OPNET: Optimized Network Engineering Tool (OPNET) is currently a part of Riverbed, an end to end solution provider for application, infrastructure and network monitoring [465]. OPNET is a network simulation tool capable of simulating heterogeneous networks while running various simulation protocols simultaneously. OPNET also provides support for WSNs and allows network customization and scalability. zigbee based networks are supported in this simulator along with customization options for zigbee coordinator, zigbee router and zigbee end devices [466]. Furthermore, mobile device can be included in the network and several network parameters can be evaluated at global, network or node level.

4) QualNet: QualNet communications simulation platform offers a testing, and training package which allows implemention of real-world networking scenario. It mimics real communication network and offers authentic and reliable network behaviour. The software provides a graphical design for the effective network formation with click and drag facilities and stack customization options [467]. Simulator also provides statistical graphing tools which is used to formulate reports and produce customized graphs using the simulation data received from the network analyzer [467]. Support for wireless ad-hoc sensor networks is included where global parameters can be set to IEEE802.15.4 radio and IEEE802.15.4 MAC for the entire network [468]. Network parameters are customizable and performance of the network can be visualized and evaluated using simulators 3D visualizer and network analyzer [468].

\section{Industrial services providers}

Due to the high cabling deployment and maintenance cost, it is expected that the IWSNs will see a great boost in the near future [33], [34]. It is the primary reason, why many leading industrial process control and automation service providers have started investing in IWSN based industrial solutions. With the prediction of IWSNs market soaring as high as \$3.795 Billion by end of year 2017 [34], many new wireless solutions for industrial automation and process control have been launched. This trend, adopting IWSNs for industrial solutions, can be observed in several renowned companies offering services in the automation and process control. A significant number of such companies have started offering industrial wireless networks based solutions to establish communication links between the central control unit and the industrial equipment. Table X presents a list of key companies offering services in industrial monitoring and control systems using IWSNs.

\section{MAC LAYER OPTIMIZATION AND MAC SCHEMES}

Over the years many solutions for IWSNs were also proposed by the research community. These proposed schemes involved improvements in reliability, real-time operability, network life enhancement and deterministic network formation. Most of these researches focused on MAC layer optimization, primarily because MAC layer handles two most important tasks, controlling nodes access to the wireless medium and managing the use of radio. Efficient channel access improves both reliability and real-time data delivery and offers better congestion control, whereas efficient use of radio improves network lifetime [36]. In this section, a detailed review of MAC protocols is presented to offer insight of current research trends in MAC optimization for IWSNs. 


\section{A. Classification of MAC Protocols and IWSN MAC Develop-} ments

During the last few years the design objectives of MAC protocols have experienced a significant change [187]. Earlier researches sacrificed throughput and reliability for extended network lifetime [44], [186]. However, for IWSNs, the energy efficiency in MAC protocols has become a secondary objective, where the network can no longer rely on best effort data delivery services [38].

To label the MAC layer developments according to industrial application requirements, an extended taxonomy of MAC protocols is created. The taxonomy labels noteworthy MAC developments according to their suitable application area in industry. The taxonomy of MAC protocols is presented in Fig. 11, which categorizes MAC based developments with respect to channel access scheme, target application area, latency, reliability bounds and single channel and multichannel attributes. Furthermore, a classification of MAC protocols is also represented in Table XI which classifies notable MAC protocols based on communication priority, latency and area of application.

Over the time a large number of MAC protocols are being presented and one can find an exhaustive list of such protocols in [36], [38], [41], [45], [188], [189]. In [189] MAC protocols are classified in four categories. The classification is based on the medium access methods, hence categorizing MAC protocols in to random, periodic, slotted and hybrid access schemes. In [36] the MAC protocols are also classified in four categories namely asynchronous, synchronous, slotted and multi-channel. Each of these categories has their own significance and offer unique benefits. Asynchronous protocols can run on very low duty cycle, a desirable trait for longer lifetime but the efficient communication between the nodes and congestion control are major challenges. Communication challenges present in asynchronous MAC protocols are suitably resolved in synchronous protocols but in these protocols channel congestion and collision avoidance remains an issue. Slotted schemes resolve the issue of channel congestion but channel utilization in such cases is relatively low. The Multichannel schemes take benefit of full potential of wireless motes by implementing both TDMA and Frequency Division Multiple Access (FDMA) to improve the channel capacity. Apart from these classifications, there are many protocols that target MAC layer optimization in both TDMA based and CSMA/CA based channel access schemes.

In our classification, the MAC protocols are distributed in: Contention (CSMA/CA) based schemes, TDMA based schemes, multi-channel schemes and priority enabled schemes. Each of these is listed as follows.

1) Contention based MAC protocols: CSMA/CA based medium access protocols fail to offer deterministic behavior which compromises their effectiveness in critical industrial applications [190]. Hence, industrial applications with less stringent deadlines can only be suitable for CSMA/CA based medium access protocols. In [191], Markov chains are used to model relations of packet transmission, packet delay, and en- ergy consumption. Using this model, a distributed adaptive algorithm is derived to minimize power consumption along with improving packet reception probability and delay constraints. In [210] authors present a predictive wakeup mechanism in asynchronous duty cycling to reserve energy. In [209], authors present a sparse topology and energy management technique which wakes the radio from deep sleep state without the use of low power radio. Some other contention based schemes include [192], [193], [194], [195], [196].

Furthermore, to provide an extensive classification of the MAC protocols, in the MAC taxonomy presented in Fig. 11, the contention based schemes are classified in six categories namely: A) low duty cycle with extended lifetime, B) contention based low latency schemes, C) periodic contention based sustainable networks, D) contention based bounded deadtime communication, K) Throughput enhancement using contention based multichannel access and L) delay sensitive multichannel emergency access. The labels A, B, C, D, K and L are same as used in the taxonomy to maintain symmetry. The contention based MAC protocols falling in any of these categories are presented in Table XI along with other attributes of these protocols.

a) Summary and insights: In IWSNs, contention based channel access schemes has very limited use in the process control due to the non-deterministic nature. However, the CSMA/CA allows the nodes, freedom to communicate whenever needed and hence serves as a suitable mechanism to offer improved network lifetime in non-critical monitoring and data accumulation applications. Over the years plenty of CSMA/CA based MAC protocols have been introduced. The primary target as observed was to extend the network lifetime by introducing suitable sleep mechanism. Since this communication offered nodes to wake-up only when transmission was necessary so the protocols like STEM, STEM-B [204], PW-MAC [205] offered a suitable solution for monitoring applications.

Another and less frequently used attribute of CSMA/CA based MAC protocols was suitable reduction in the communication delay in the less congested networks. Some MAC Schemes like schemes like SIFT [220], Q-MAC [225], D-MAC [222], T-MAC [223] exploited this attribute to offer low latency in communication delay. Nonetheless, due to the contention based access, the deterministic behaviour can still not be ensured. To incorporate deterministic behaviour, slotted (BMAC [207]) and scheduled (CC-MAC [206]) CSMA were also proposed.

2) TDMA based MAC protocols: TDMA based MAC protocols serve more efficiently in ensuring reliability and latency bounds. It is for the same reason, TDMA based MAC protocols are considered more suitable for industrial applications. However, TDMA based schemes do require time synchronization and optimal TDMA scheduling is a NP-hard problem [102], [197]. In [197], authors have proposed two heuristic algorithms to solve the schedule minimization problem and ensured packet delivery. Authors have also evaluated upper bounds for these schedules as a function of total packets generated in the network. In [198], [199], authors further improved the results in [197] and showed how their work 
outperforms [197]. In [198], authors considered harsh dynamic environment but failed to offer guaranteed data reliability. In [199], authors improved reliability in harsh control environments and formed hypergraph to increase scheduling flexibility. Moreover, two schemes were also presented in this paper namely dedicated scheduling and shared scheduling and were applied to wireless sensor and control networks for performance evaluation. Another TDMA based scheme, ShedEx is introduced in [102]. This paper extends the concept of reliability improvement by repeating most rewarding slots along with a scheduling algorithm to guarantee certain specified reliability.

With respect to the taxonomy presented in Fig. 11, the TDMA based schemes are further divided in five subcategories (labelled E, F, G, M and N) depending on suitability for specific area of application. The TDMA based protocols classified in these subcategories are listed in Table XI.

a) Summary and insights: The implementation of TDMA based communication was introduced to ensure guaranteed channel access, hence eliminating the uncertainty introduced by CSMA/CA based channel access schemes. One such examples is the modification of IEEE802.15.4 for industrial applications in the form of IEEE802.15.4e which introduces TDMA based communication. Since the incorporation of TDMA reduces the uncertainty in WSNs, it can be used for the control application which require periodic feedback. It is worth noticing that IEEE802.15.4e LLDN [20] offers a suitable solution for regulatory and supervisory control applications. TDMA-MAC [200] is another protocol which ensures low latency using TDMA based communication to support feedback control systems. However, some other protocols using TDMA might not be suitable for control applications due to the introduction of long delay among two communications of an individual node. Therefore, protocols like LMAC [201], LEACH [202] and LEACH-C [202], although using TDMA, are only suitable for monitoring applications. Multi-channel TDMA schemes like ALERT [203] and T-Opt coverage [204] offer suitable reliability and latency assurance to meet the requirements of open loop control applications. Furthermore, these two schemes (ALERT and T-Opt)where work in single channel, can also benefit from the multichannel, which can be exploited to communicate to a larger number of nodes in a given time. The other TDMA based protocols which introduce priority based communication are discussed in detail in Section VIII-A(4) due to the overlapping nature of these schemes.

3) Multi-channel MAC: Use of multichannel in TDMA based MAC protocols enables improved medium utilization and offer extended features in IWSNs. In last couple of years a notable trend in multichannel MAC solutions can be seen. In [204], authors present multichannel, TDMA based source aware scheduling scheme for static networks. The algorithm benefits from multiple channels but fails to guarantee reliability. In [205], authors extended the ShedEx scheme to multichannel scenario by introducing scalable integration in existing scheme. Authors also claim to cut latencies around $20 \%$ in TDMA schedules from ShedEx. In [206], authors propose a Regret Matching based Channel Assignment algorithm (RMCA), to reduce multichannel overhead. In this paper authors investigated multichannel transmissions and used simulations and hardware implementation to demonstrate performance improvements and complexity reductions respectively. An analytic approach to model and analyze multiple channels is presented in [207]. The affirmation of model accuracy is established from numerical and simulation results. Moreover, multi-level priority for packet transmission sequence is also established.

Other multichannel schemes categorized from $\mathrm{K}$ to $\mathrm{P}$, in MAC taxonomy, Fig. 11, are listed in Table XI along with the salient features of these protocols.

a) Summary and insights: Use of multiple channels in IWSNs offer notable benefits including diversity, throughput enhancement, network scalability, optimized scheduling, on demand channel access and improved network control information. Since, in an industrial process, multiple applications can co-exist, the use of multiple-channels can introduce appropriate control in handling diverse data using parallel data streams. Apart from this, hybrid schemes with both TDMA and CSMA/CA based channel access, to support diverse traffic types, can be facilitated in parallel without introducing conflicts. RL-MMAC [244] and DSME [20] are two hybrid schemes which benefit from multiple channels to facilitate diverse traffic types. Both schemes facilitate both periodic and on-demand communication. DSME particularly focuses on improving the data rates and hence often compromises the delay constraints. RL-MMAC on the other hand offers low latency. MMSN [220], Y-MAC [222], DMC [242] are some of the CSMA/CA based multiple channel schemes which due to the presence of longer delay between the consecutive transmissions limits their scope for low latency process control. Whereas Hy-MAC [223], T-opt [204], ALERT [203] and some others introduce TDMA based channel access for collision free communication for more sensitive traffic.

4) Priority Enabled MAC protocols: In most of the industrial processes, generated information in some cases is more critical than the rest hence should be prioritized above the rest of the communication. The priority based communication in IWSNs facilitates the communication of high priority traffic by providing adaptive channel access. Some of the proposed work in this domain includes [30], [54], [55], [207], [208]. In [207], authors presented a priority enabled MAC to prioritize messages with high information content. The protocol supports deadline requirements for feedback control systems but assumes full duplex communication which is not true in IWSNs. A priority enhanced MAC protocol for critical industrial applications is presented in [55]. In this protocol the traffic in an industrial communication network is divided in four groups and the protocols allows the high priority traffic to overtake the low priority traffic bandwidth. The paper presents performance analysis and evaluation of the protocol through experimental implementation. In [30], a priority enabled MAC is defined in which priority is assigned on the basis of arbitration frequency allocated to individual users. The protocol is evaluated using discrete time Markov chain model and guaranteed access of the highest priority user is assured.

A classification of certain other priority enabled MAC pro- 
tocols is also presented in Table XI, where the priority mechanism along with the salient features of these protocols is listed.

a) Summary and insights: The diverse nature of industrial applications introduces a wide variety of sensory data to be accumulated at the control centre. Since various industrial processes run simultaneously in an industrial environment and cannot be distributed geographically. Therefore, it is much more obvious that communication link must relay data from regulatory control, supervisory control, open-loop control alerting and monitoring applications through the same wireless link. Under such circumstances, the priority based communication offers a significant improvement in network efficiency by communicating the traffic according to sensitivity levels. However, in IWSNs, the priority based communication is not thoroughly evaluated and very few protocols offer priority based communication. Nonetheless, the significance of the priority based communication cannot be undermined. Priority based communication also increases the diversity of the network by allowing sensory data from different applications to be communicated to the control centre without affecting the critical processes thus improving the efficiency of the entire network.

\section{B. Extended Classification of MAC Protocols}

The taxonomy presented in Fig. 11, provides a much wider and in-depth view of possible MAC layer developments, and allows to classify different schemes presented over the years into one of the sixteen possible categories. The taxonomy is developed to assist in the evaluation of the basic requirements of a protocol and its much accurate characterization into one of various application areas in industrial environments. Furthermore, MAC protocols presented in research literature over time are also characterized into the categories specified by the MAC taxonomy. The classification of notable MAC protocols based on the presented taxonomy are listed in Table XI. Although, the classification of various protocols is discussed earlier on case to case basis, in general, the presented classification can be broadly distributed in single channel and multi-channel schemes, with further distribution of contention based, slotted and hybrid access schemes. Further subdivision in contention based, slotted and hybrid schemes maps to a particular application domain, dealing with one of the six industrial systems, discussed in II-(A). The MAC taxonomy also lists the objectives of WSNs and IWSNs and defines the bounds on key parameters.

The protocols listed in Table XI, are classified using the presented MAC taxonomy to evaluate their suitability in IWSNs. For instance, MAC protocols including STEM [209], PWMAC [210], CC-MAC [211], B-MAC [212], SyncWUF [213], TrawMAC [214], TICER [214], S-MAC [55], DS-MAC [217] and few others can be used in the monitoring applications. However, out of the above listed protocols, STEM, STEM$\mathrm{B}$ and PW-MAC are more suitable for the applications with asynchronous communication requirements whereas almost all of the rest are suitable for periodic monitoring applications. Similarly, LAMA [218], PriorityMAC [55] and EQ-MAC
[219] offer prioritized access which make these protocols suitable for the applications where the industrial system is handling more than one type of traffic. Usually these protocols are suitable for the systems dealing with supervisory control and alerting systems, where, depending on the traffic type the priority is assigned. To diversify the monitoring, control, and emergency communication in IWSNs, multi-channel MAC schemes were also presented over the years. The multi-channel schemes like MMSN [220], TMCP [365] and Y-MAC [222] can be used in the asynchronous monitoring applications with relatively larger networks. Whereas schemes like HyMAC [223] and FDP-MAC [224] can be put in use for time sensitive applications. A detailed list of various multi-channel schemes, along with the protocol characteristics and latency details are listed in Table XI.

\section{Network Layer Developments}

Network layer plays an important role in real-time and reliable communication of information in IWSNs. A large number of protocols have been proposed to meet routing requirements in diverse applications in conventional and industrial WSNs. Over the years, routing protocols are proposed to improve certain key attributes of a network. Some of the key performance metrics and network attributes optimized by routing include network lifetime, latency, throughput, reliability, energy efficiency, robustness Packet Reception Rate (PRR), scalability and algorithm complexity [380], [382]. Extensive list of routing protocols addressing one or more of the listed performance metrics can be found in [378], [379], [381], [382], [284], [376], [377].

Routing protocols due to significant variations in the underlay architecture and applications of IWSNs, can be classified in multitude of ways. A few attempts have been made to classify routing protocols based on significant features and some of these classifications can be found in [376], [377], [382], [381], [380]. In this section, an extended classification of routing protocols is presented where the significance of various classes of routing protocols is discussed.

The extended classification of routing protocols is presented in Fig. 12. The overall classification of the routing protocols is divided in six sub-categories, where each of these categories is discussed in detail. The created taxonomy of the routing where distributes the protocols into multiple categories, it also labels each subcategory with respect to performance metrics to highlight the focus of the created category and its suitability for different applications. In Fig. 12, colour coding is used to label the routing subcategories in accordance with the performance optimization parameters listed in the figure. Three performance optimization parameters are considered latency, energy efficiency and scalability. Based on these performance metrics and the potential of the routing subcategories each routing subcategory is appropriately labelled.

\section{A. Flat}

In flat routing protocols, all nodes are assigned with equal role and functionality which reduces the overhead and offers simplistic rules to manage entire networks [377]. The 
TABLE XI: Salient Features of Selected MAC Protocols

\begin{tabular}{|c|c|c|c|c|c|}
\hline Protocols & $\begin{array}{l}\text { Channel Access } \\
\text { Scheme }\end{array}$ & $\begin{array}{l}\text { Frequency } \\
\text { channels }\end{array}$ & $\begin{array}{l}\text { Application } \\
\text { as per Fig. } \\
11\end{array}$ & Latency & Priority \\
\hline STEM [209], PW-MAC [210] & Contention based & Single & A & High & No \\
\hline STEM-B [209] & $\begin{array}{l}\text { Contention based } \\
\text { with synchronized } \\
\text { paging }\end{array}$ & Single & A & High & No \\
\hline $\begin{array}{l}\text { SIFT [225], STEM-T [226], } \\
\text { D-MAC [227], T-MAC (FRT- } \\
\text { S) [228], ADCA MAC [229], } \\
\text { Q-MAC [230], D-S Adap- } \\
\text { t [231] }\end{array}$ & Contention based & Single & B & Medium to low & No \\
\hline CCMAC $[211]$ & Scheduled CSMA & Single & $\mathrm{C}$ & High & No \\
\hline B-MAC [212] & $\begin{array}{l}\text { Slotted contention ac- } \\
\text { cess }\end{array}$ & Single & $\mathrm{C}$ & High & No \\
\hline $\begin{array}{l}\text { SyncWUF [213], TrawMAC } \\
\text { [214], TICER [215], RICER } \\
\text { [215], S-MAC [216], DSMAC } \\
\text { [217], MS-MAC [232], Op- } \\
\text { timized MAC [233], PMAC } \\
\text { [234] }\end{array}$ & Contention based & Single & $\mathrm{C}$ & High & No \\
\hline SchedEx $[102]$ & $\begin{array}{l}\text { CSMA/CA contention } \\
\text { based scheduling }\end{array}$ & Single & $\mathrm{D}$ & Low & No \\
\hline DW-MAC [235] & contention based & Single & $\mathrm{D}$ & Low & No \\
\hline $\begin{array}{l}\text { LLDN [20], TDMA-MAC } \\
\text { [200] }\end{array}$ & TDMA & Single & $E$ & Low & No \\
\hline $\begin{array}{l}\text { LMAC [201], LEACH [202], } \\
\text { LEACH-C [202] }\end{array}$ & TDMA & Single & $\mathrm{F}$ & High & No \\
\hline LAMA [218] & TDMA & Single & G & Low (selected data) & Q \\
\hline NAMA [218] & TDMA & Single & G & Low (selected data) & $\mathrm{R}$ \\
\hline PAMA [218] & TDMA & Single & G & Low (selected data) & $\mathrm{S}$ \\
\hline EQ-MAC(CMAC) [219] & TDMA & Single & G & Medium to low (selected data) & $\mathrm{T}$ \\
\hline PriorityMAC [55] & TDMA & Single & G & Low (selected nodes) & $\mathrm{U}$ \\
\hline Wise MAC [236] & Hybrid & Single & $\mathrm{H}$ & High & No \\
\hline TRAMA [237] & Hybrid & Single & $\mathrm{H}$ & Medium to High & No \\
\hline FSC [10], SSA [10] & Hybrid & Single & $\mathrm{H}$ & Low & No \\
\hline WirArb [30] & Hybrid & Single & $\mathrm{I}$ & Low (selected nodes) & $\mathrm{V}$ \\
\hline Z-MAC [238] & Hybrid & Single & $\mathrm{J}$ & Medium to low & No \\
\hline PARMAC [239] & Hybrid & Single & $\mathrm{J}$ & $\begin{array}{l}\text { Low to medium (Intragrid) } \\
\text { High (Intergrid) }\end{array}$ & No \\
\hline $\begin{array}{l}\text { HMAC [240], EQMAC (CA- } \\
\text { MAC) [219] }\end{array}$ & Hybrid & Single & $\mathrm{J}$ & Low & No \\
\hline GANGS [241] & Hybrid & Single & $\mathrm{J}$ & Medium & No \\
\hline MMSN [220], Y-MAC [222] & CSMA/CA & Multi-channel & $\mathrm{K}$ & Medium & No \\
\hline TMCP [365] & CSMA/CA & Multi-channel & $\mathrm{K}$ & High & No \\
\hline DMC-Allocation [242] & CSMA/CA & Multi-channel & $\mathrm{L}$ & Low & No \\
\hline $\begin{array}{l}\text { ALERT [203], T-opt Coverage } \\
\text { [204] }\end{array}$ & TDMA & Multi-channel & M & Medium to low & No \\
\hline $\begin{array}{l}\text { HyMAC [223], FDP-MAC } \\
{[224]}\end{array}$ & TDMA & Multi-channel & M & Low & No \\
\hline $\begin{array}{l}\text { SchedEx (M-C) [205], DMP } \\
\text { [243] }\end{array}$ & TDMA & Multi-channel & $\mathrm{N}$ & Medium to low & No \\
\hline RL-MMAC [244] & Hybrid & Multi-channel & $\mathrm{P}$ & Medium to low & No \\
\hline DSME [20] & Hybrid & Multi-channel & $\mathrm{P}$ & Medium (12xhigh data rates [245]) & No \\
\hline \multicolumn{6}{|c|}{ Q: Priority evaluation in two hop neighbourhood, with link activation access provided to priority node } \\
\hline \multicolumn{6}{|c|}{ R: Self and neighbour Priority evaluation by sensor nodes to determine the priority of access to the slot } \\
\hline \multicolumn{6}{|c|}{ S: Prioritized link activation to destination nodes } \\
\hline \multicolumn{6}{|c|}{$\begin{array}{l}\text { T: The scheme classifies the gathered data in to queues based on the importance and the high priority queue gets the privileged access to the } \\
\text { channel }\end{array}$} \\
\hline \multicolumn{6}{|c|}{ U: Four level Priority is established with high priority node given the access to highjack the timeslot of the low priority node } \\
\hline \multicolumn{6}{|c|}{$\begin{array}{l}\text { V: An arbitration decision period is run and Frequency polling is used where each node is pre-assigned a frequency based on its priority. Based } \\
\text { on the frequency polling in arbitration phase node with highest priority gets access to first time slot in arbitration execution period and so on } \\
\text { where nodes with lower priorities have to wait till all the higher priority nodes have communicated }\end{array}$} \\
\hline
\end{tabular}




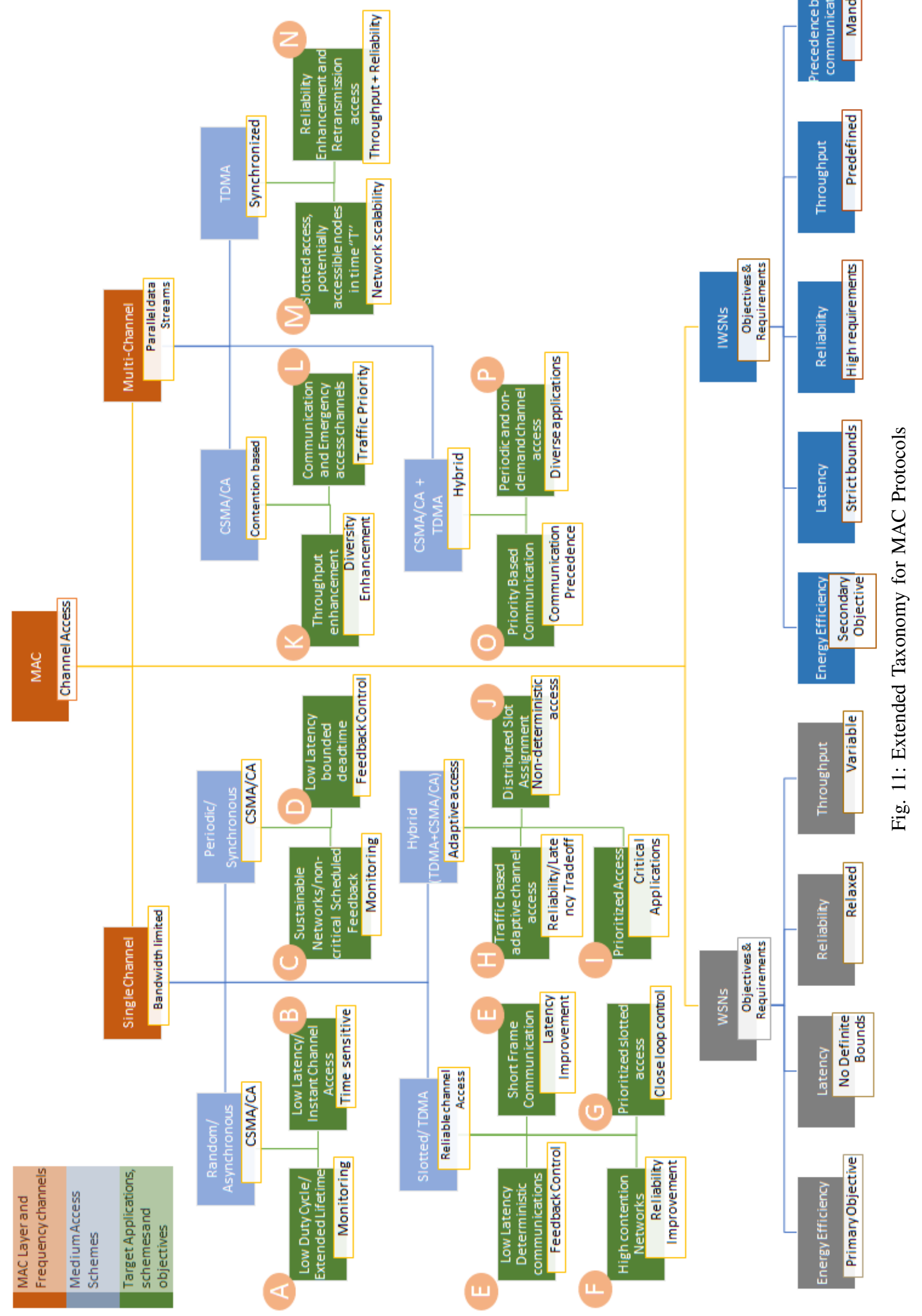


complexity of such protocols is relatively low and they are suitable for the data gathering applications. The flat architecture reduces the network complexity, however, it also adds longer delay in larger networks. Some of the flagship routing protocols in this category include WRP [383], TBRPF [384], TORA [385], Flooding [386], Gossiping [387] and ZRP [388].

\section{B. Hierarchical}

Hierarchical routing protocols benefit from the versatility of nodes in the network. The use of special purpose nodes in the networks reduces the added delay in flat routing protocols [378], [381]. Due to the hierarchical attributes of the network, a fairly optimized low latency 2-hop communication can be established in the network, where the sensing nodes relays information to cluster-head (a special purpose node capable of communicating directly to sink) which then communicates this information to sink. Hierarchical routing protocols due to their unique attributes, offer reduced delay, hence offering a suitable solution for time critical industrial applications. A classification of hierarchical routing protocols can be found in [378], [381] whereas some of the examples of hierarchical protocols are: LEACH [389], TEEN [390], PEGASIS [391], APTEEN [431], MIMO [393], HPAR [394], NHRPA [395] and DHAC [396].

\section{Geographic}

Geographic or location based routing protocols use geographical placement of nodes to evaluate optimum path for routing. Usually these protocols rely on the built-in positioning systems embedded in the nodes. With the knowledge of accurate location of nodes geographical routing protocols formulate optimal path from source to destination, however, the use of positioning systems in nodes adds to the cost and also limits the use of such protocols in covered locations. Some of the location based routing protocols include TTDD [397], COUGAR [398], GEM [399], GEAR [400], IGF [401], SELAR [402] and OGF [403].

\section{Gradient}

Gradient based routing protocols use gradient cost field establishment to route data from farther ends of the networks to the sink. The concept of gradient cost field establishment is taken from a natural phenomenon where water flows from higher grounds to the valley. Similarly, in gradient based routing protocols data propagates in a direction where it finds minimum cost. Each node maintains a routing table with at least the information of one least cost neighbour. The cost field at each sensor node can be defined in terms of hop count, energy consumption, delay, link quality, node energy etc. Any one or a combination of above described parameters can be used to establish Gradient cost model. In gradient based routing, the cost field can be established as a function of any of the QoS attributes which can optimize the network for desired quality metric. Selection of right quality metric can lead to an application specific and optimized routing solution. Some of the flagship gradient based routing protocols include GBR [405], GRAB [118], GRACE [404], PC-GRACE [106], SGF [406] and RRP [407].

\section{E. Cluster based routing}

In cluster based routing protocols the network is divided into clusters. Each cluster formulates a small network, where one of the nodes in the cluster acts as a cluster-head/coordinator. This node is responsible for ensuring the affiliation of nodes in the cluster, accumulation of data from these nodes and onward communication of the accumulated information to the sink[377]. Due to the distributed solution, cluster-based approaches offer delay minimization, redundancy reduction, robustness and efficient intra-cluster and inter-cluster communications among nodes. Some of the cluster-based routing protocols include LEACH [389], TEEN [390], PEGASIS [391], Re-cluster-LEACH [408], SOP [409], CHR [410], and IDSQ [411].

\section{F. Mobility aware routing}

Mobility aware routing considers the time to time change in the position of the sink or certain mobile nodes and updates the routing paths accordingly. It is always hard to ensure reliability, energy efficiency and communication overhead minimization within the mobile networks. While some routing protocols consider the scenario of mobile sink, others do allow the mobile nodes to be part of the network. For mobile sink based protocols although the sensors have to update the routing path however if sink movement is strategically planned, it resolves sink hole problem (heavy use of sensors located around static sink) [378]. Some of the proposed protocols to handle mobility in the networks include MIP [412], IEMF [413], Joint mobility and routing [414], DataMULEs [415], SEAD [416] and proxy tree-based data dissemination [417].

\section{G. Multipath routing}

Multipath routing allows multiple streams of information from source to sink. Multipath routing protocols exploit the existing multi-paths in the network to offer improved reliability of the data. Although multipath routing protocols are less energy efficient and multiple copies of same data can cause network congestion, however, accurate data reception and reliability of the data significantly increased by implementing multipath routing. The path selection in multipath routing is quite similar to single path routing where instead of the selection of single most suitable path, multiple paths in order of their suitability are selected for routing of data. Some of the multipath routing protocols include GRAB [418], TBRPF [384], TORA [385], TTDD [397], MIMO [393] and HMRP [419].

\section{H. Proactive}

In proactive routing protocols, each node is aware of the data flow path to the sink hence route discovery delay in such protocols is eliminated. This allows proactive protocols to offer improved latency in data communication and can be used for real-time data delivery. The basic requirements of low latency in such protocols urges dissemination of periodic updates. The periodic update messages disseminated throughout the network allows the path reestablishment in case of change in topology 
[382]. Since frequent updates are required in proactive routing protocols, the communication overhead is relatively much higher. Some of the examples of proactive routing protocols include 2-Tier data dissemination [420], SDG [421], SPIN [422], CRP [423], Leach [389] and SOP [409].

\section{Reactive}

Reactive protocols in opposition to proactive protocols, establish on-demand communication and require very little information about the route at individual node level. Since the intermediate nodes do not have to make routing decisions therefore, there is no need to keep routing tables and information of the neighbouring nodes [382]. Furthermore, the overall efficiency of reactive protocols is relatively higher due to less communication overhead. However, the lack of information of routing path and on-demand path establishment adds additional delay to the communication of data. This delay is usually referred as path acquisition delay [382], [427]. Some of the traditional reactive routing protocols include TEEN [390], COUGAR [398], CADR [424], EAR [425] and RR [426].

\section{J. Hybrid}

Hybrid schemes benefit from both the features of proactive and reactive routing protocols. The communication region influences the use of proactive and reactive routing. Most of the hybrid protocols use proactive routing locally and reactive routing inter-locally [382]. Due to the optimal selection of the routing, the hybrid protocols offer reduced latency, overhead reduction and scalability within the network. Some of the hybrid protocols include GBR [405], PEGASIS [391], SPEED [428], MSRP [429], JARA [430] and APTEEN [431].

\section{K. Query based}

In query based routing protocols the communication between the source and destination is initiated by a query request circulated by the destination node. The destination node (sink) if needed circulates a request for a specific data (based on location or specific attributes of the sensed data). Any node having the data of interest responds to the request with appropriate sensory data. The query based data routing algorithms minimize the unnecessary data communication thus avoiding flooding of data in the network resulting in extended network lifetime [377]. The query based algorithms are more suitable for delay tolerant applications due to the added delay from query request to delivery of data. The delay is further increased in reactive query based algorithms. Some of the query-based routing protocols include RR [426], DD [432],COUGAR [398], ACQUIRE [433], SPIN-PP [434] and EQSR [435].

\section{Data centric}

In data-centric routing protocols, when a sensory data is forwarded from the source node, the intermediate nodes can perform the data aggregation in the original data packet. This allows efficient aggregation of data and reduces the communication overhead. However, due to the manipulation in the source packets, the identifiers can be altered due to which the received information cannot be individually identified with respect to the sources. Some of the representative protocols in this category include SPIN [422], COUGAR [398], RR [426], ACQUIRE [433], DD [432] and EAR [425].

\section{Address centric}

Address-centric protocols differ from data-centric with respect to data delivery mechanism. In address-centric routing protocols data is transmitted individually from source to sink. The information from each sensor node is transmitted independently to keep the identity of originating entity intact. Unlike data-centric, this type of protocols gives relatively higher importance to locality of the originating data. Some of the address-centric protocols include dream [436], LAR [437], GRACE [404] and GRAB [418].

\section{N. Negotiation based}

Negotiation based routing protocols use meta-data negotiations to reduce the redundant transmissions [382]. These protocols focus on energy efficiency and hence communication is limited only to the occasions when requested. Negotiation based protocols usually follow a three-stage cycle for information communication. In case of an event, sensor node advertises event based sensory information in first stage where advertisement packet is broadcasted. Seeing the advertisement packet, if any nodes requires the advertised data, it will send a request packet indicating its interest in the data. In the third stage the data is transmitted from the sensor node to interested node completing three stage-cycle: ADV-REQDATA [382]. Negotiation based protocols prioritize operational efficiency and energy conservation at the cost of added delay and therefore can be used in monitoring applications. Some of the selected negotiation based routing protocols include SPIN [422], SPIN-PP, SPIN-RL [434], DD [432], VGA [438] and SAR [439].

\section{O. Homogeneous}

The routing protocols are sometimes divided based on the nodes in the network. If all the nodes in the network are same with respect to the hardware, size, battery, energy supply, energy harvesting, radio and transmission powers, the nodes are considered as homogeneous nodes [377]. The routing approaches used for homogeneous sensing networks are referred as homogeneous routing protocols. Due to the similar attributes of the nodes, homogeneous routing protocols treat all nodes equally. Some of the homogeneous routing protocols include PEGASIS [391], TEEN [390], LEACH [389], COUGAR [398], SPIN [422], DD [432] and SPEED [428].

\section{P. Heterogeneous}

Unlike homogeneous routing protocols, heterogeneous routing protocols are capable of handling more diverse networks. Heterogeneous routing protocols takes in to consideration the 


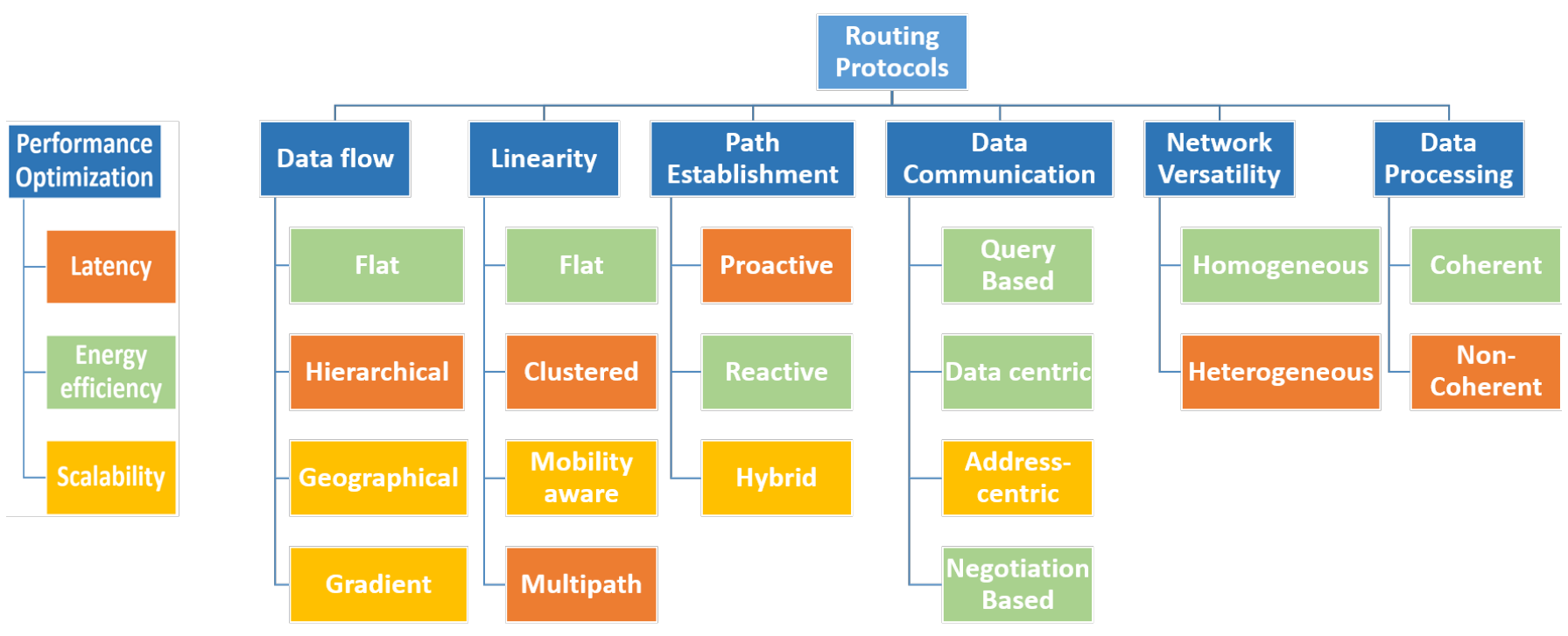

Fig. 12: Classification of Routing Protocols

versatility of the networks and are more suitable for networks with heterogeneous sensing nodes [377]. The heterogeneous routing benefits from the special features of the nodes in the network, where the nodes with extended battery, range, bandwidth can work as coordinator, cluster-head or aggregator to facilitate network management and efficient communication of information from primitive nodes. Some of the example of heterogeneous routing protocols include SOP [409], CHR [410], IDSQ [411] and CADR [424].

\section{Q. Coherent and non-coherent routing}

In coherent data processing based routing protocols, the sensor nodes transmit the data with minimum processing. This type of routing poses minimum processing load on the sensor nodes. The sensor nodes use primitive algorithms for time stamping and redundancy reduction cite napantazis. This allows nodes with low processing specifications to work effectively without overburdening them. In Non-coherent routing, the senor nodes locally process the data and then it is forwarded to aggregators for onward processing. Data processing in noncoherent routing goes through three phases [382].

- Target detection, data collection and pre-processing

- Membership declaration

- Central node election

In first phase the detection of an event, collection of events sensory data and pre-processing of data is completed. In the second phase, the node selects to participate in the cooperative function and informs the neighbouring nodes. In last phase, a node is elected to perform further processing on the sensory information and apply more extensive compression, encryption, and aggregation on the received information [382], [377]. It is observed that the localized processing in non-coherent processing based routing protocols offer better scalability and reduced delay compared to coherent processing based routing. Examples of coherent and non-coherent processing based routing include SWE and MWE [440], [441].

\section{EnERgy Sources AND ENERgy Harvesting IN IWSNS}

The current industrial equipment is developed to last for months, even year(s) without a replacement or maintenance. This advancement in technology allowed to cut down regular maintenance costs in industries. However, the network lifetime of IWSNs must also be improved in order to allow smooth operation of the plant by avoiding battery replacements and redeployment of nodes, until the regular maintenance of the equipment is called. Due to the extended lifetime requirements, energy harvesting in IWSNs has gained much importance. Many schemes are proposed that can add up in the lifetime of the network by harvesting energy from the available sources in the dynamic industrial environment. A number of techniques can be used to capture energy. Photovoltaic (PV), wind, thermal, biochemical, vibrational, pressure, nuclear, microwave, magnetic resonances, and Radio Frequency (RF) are some of the options for energy harvesting techniques [39], [248], [249], [250], [251]. The details of these energy harvesting techniques and energy sources are listed as follows.

\section{A. Radiant Energy}

Radiant energy plays a significant role in energy harvesters and is one of the most widely used energy sources. Radiant energy harvesting can be divided into solar, RF and infrared. 


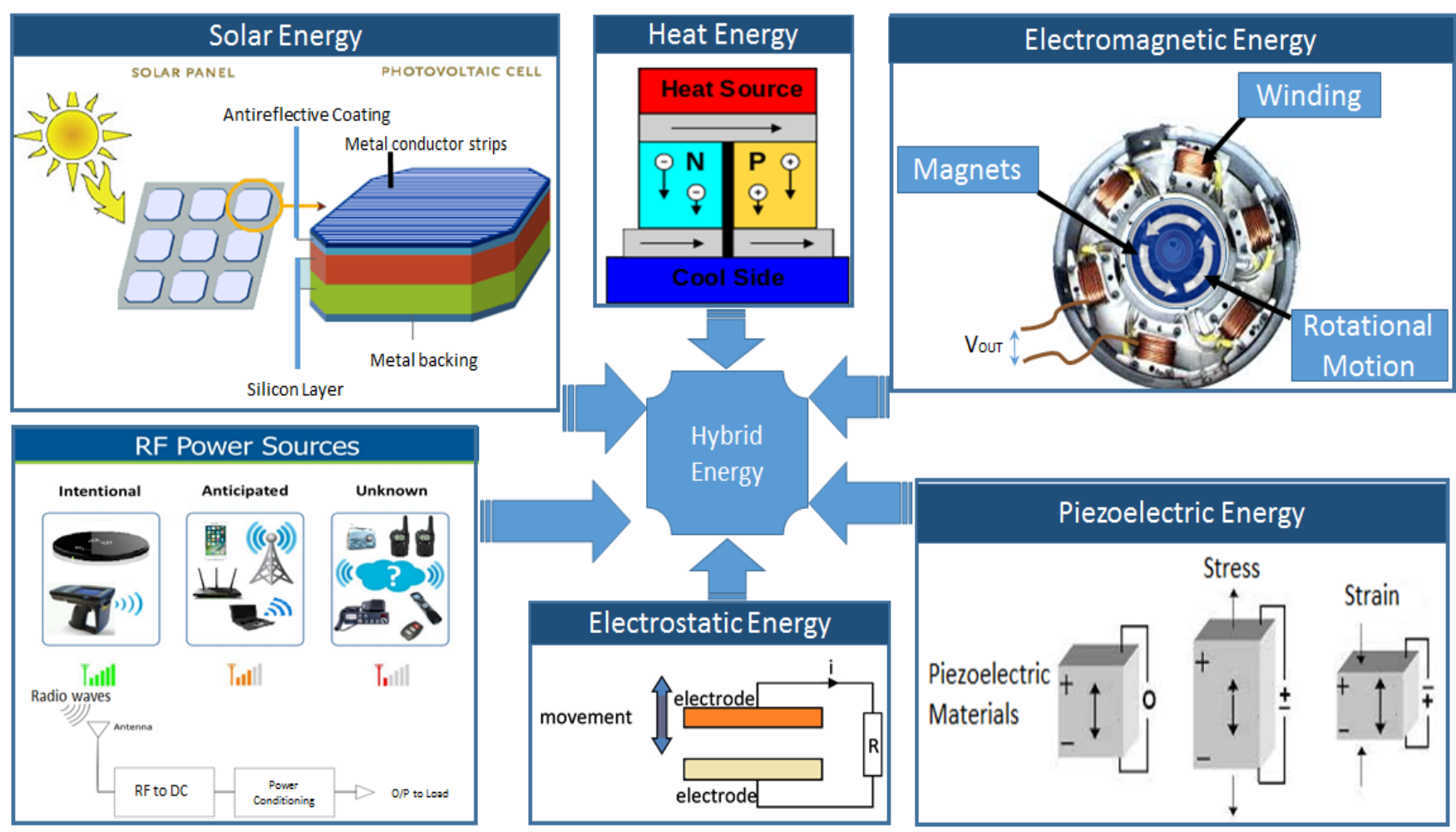

Fig. 13: Energy harvesting techniques in IWSNs

1) Solar: A wide variety of applications use PV for outdoor/indoor energy harvesting. Energy harvested by PV varies significantly, depending on the indoor and outdoor environment and lighting conditions.

The use of solar/illumination for outdoor/indoor applications in IWSNs has been used for variety of applications [46], [39], [252], [253]. Since the overall energy requirements are relatively low in case of IWSNs, relatively small size PVs can be used to meet the purpose. In some cases, for outdoor applications the hybrid photovoltaic/temperature (PV/T) cells are also used to offer improved efficiency. For outdoor environments, the solar energy is termed as an infinite source of energy, but the energy harvested on different days on different times with different weather conditions can vary tremendously [47], [254]. Hence, the energy harvested from the solar cells is termed as uncontrollable and less predictable due to the randomness in its behaviour. The indoor light harvesting, however, is usually more predictable as the sources of illumination work during certain predefined hours. Furthermore, in industrial environments certain luminous levels are maintained to ensure effective work environments. A careful survey of lighting conditions in different regions in the indoor industry floors and points of interest can give a better estimate of energy harvesting potential in such environments [255], [256]. Indoor, although predictable, yet is less effective, as the efficiency of the indoor PV harvesters is reported to be reduced to one third [47]. The graphical representation of solar energy harvesters is presented in Fig. 13, whereas the details of energy harvesting potential of solar harvesters are presented in Table XII.
2) $R F$ : The RF based energy harvesting has gained much attention in the last decade [48], [257]. With the exponential increase in the wireless communications, and countless sources transmitting electromagnetic radiations, RF energy harvesting has become a definitive source for energy. Presently, the Wi-Fi access points, cellular base stations, WiMAX communications, TV broadcasts, and radios are emitting large amount of energy in the atmosphere.

The RF energy sources can be divided in two areas: near-field and far-field harvesting. The near-field wireless energy harvesting targets the applications where the transmitter is placed in close vicinity of the RF energy harvester, which harvests energy from the close range known transmitter. Since, in such cases, a predefined distance is maintained which makes such cases predictable and much more reliable. In the near-field energy harvesting, the efficiency is reported to be up to $80 \%$ [258]. In the far field, the energy is harvested from the different wireless communications and broadcasts taking place in the vicinity [48]. The energy is harvested from the RF/microwave radiations using a wide band antenna or array of antennas. This received energy is then rectified and transformed into power [49], [259]. It is considered as a relatively consistent and sufficient source of energy in the urban areas, however, its density per square $\mathrm{cm}$ reduces significantly in the suburbs [47].

3) Infrared energy: Infrared energy harvesting targets next generation of the PVs to harness energy from the infrared radiations emitted by the hot bodies. All hot bodies, as a power dissipation mechanism, emit infrared radiations which carry a notable amount of energy. Harvesting energy from the infrared 
radiations offers an alternate to the solar power during night. Furthermore, in the industrial environments, a lot of energy is wasted in the form of infrared radiations which can be effectively transformed in to an untapped energy source.

4) Summary and insights: The primary objective of energy harvesting in IWSNs is self-sustainability. Therefore, selection of an energy harvesting scheme is essentially motivated by the energy requirements of the sensor nodes. The energy consumption of nodes even with same hardware specifications, can change significantly depending on the frequency of communication, sleep scheduling and passive listening duration. Therefore, there are no clear boundaries for suitability of application areas of a specific energy harvesting technique and can be used in diverse applications. Nonetheless, based on the potential of various radiant energy sources and their suitability to certain application areas some recommendations are provided as follows.

Solar is one of the readily available and most frequently used energy harvesting schemes in IWSNs. Due to the abundance of this resource, applications requiring more frequent transmissions including regulatory and supervisory control can easily be powered up using PV based energy harvesting. Motes using solar based energy harvesting are equipped with a primary rechargeable battery and follow the harvest-storeuse model. It is also noted that the outdoor nature of solar energy harvesting nodes encourages larger separation between the nodes which requires more powerful radios. Some of the solar powered energy harvesting nodes include Heliomote, Prometheus, Ambimax and sunflower [39]. Although solar energy harvesting in IWSNs is widely used and is highly suitable for outdoor applications, yet the uncertainty and dependence on the weather conditions make the source unpredictable.

RF based energy harvesting can serve a potential source of energy in near field region and can provide low cost long life energy harvesting. However, RF energy harvesting produces much lower power levels in the order of microwatts even in the close vicinity of the cellular towers $(15 \mathrm{~m}-100 \mathrm{~m})$ hence, the RF based energy harvesters are only suitable near transmission towers and wireless access points in industrial environments. Infrared energy harvesting due to its dependence of low energy wave emissions by hot bodies is more suited for industrial environments with plenty of hot bodies. Boilers, incinerators, furnaces, engines, and hot bodies can serve as source of energy for the infrared based energy harvesting. Since the infrared based energy harvesting offers limited energy therefore, it is suitable for applications with less frequent transmission requirements. For such energy harvesting sources, it is also desirable to incorporate efficient power management schemes.

\section{B. Heat Energy}

Heat energy is one of the sources that is available in abundance in the industrial environments and is therefore, termed as a significant contributor in energy harvesting systems. The heat based energy harvesting relies on the heat energy produced from the mechanical equipment, structures, friction, heating chambers, heating losses, even from the electrical equipment [35]. The thermal energy harvesting systems rely on thermoelectric effect [260], where electrical energy is produced due to the temperature difference achieved between the two surfaces of different materials [261]. The principle of transforming temperature difference between two surfaces into voltage is also referred as Seebeck effect [249], [251]. Although the energy efficiency of the thermoelectric devices is relatively low but such devices offer a relatively long lifetime. Furthermore, thermoelectric energy harvesting is termed as a reliable energy source with relatively low maintenance.

1) Summary and insights: The miniature size of the thermal system allows nodes to use heat energy harvesting in wide variety of industrial applications. The use of heat based energy harvesting nodes are commonly used in the radiators and heated surfaces in industrial environments [39]. The use of thermoelectric charge is also common in the temperature control applications where the thermoelectric energy harvesting powers the feedback communication of the nodes. Furthermore, the use of heat energy harvesting nodes is also introduced in automotive industry where the engine heat and exhaust waste are used to produce thermoelectric charge [35]. While the infrared energy harvesting nodes are not bounded by the time of the day and sunshine like solar PVs and can work during the night time as well, yet the source is very limited and only serves for low duty cycle nodes with extended sleep duration.

\section{Vibration Energy}

Mechanical forces, found in abundance in industrial environments, are great sources of energy and are widely accepted in the industrial environments. Whether it is achieved through electrostatic, piezoelectric, or electromagnetic, [262], [263] this energy resource can greatly impact the lifetime of the IWSNs. The electrostatic, electromagnetic, and piezoelectric devices transform the mechanical energy into electrical energy. The working principle of these devices is listed as under.

1) Piezoelectric: The piezoelectric effect based devices use piezoelectric material which under the influence of stress or strain, are capable of producing electric charge. The produced charge is regulated to achieve a steady output. The piezoelectric systems offer advantages of high output voltages and capacitances but are relatively expensive and the coupling coefficient is also material dependent [260].

2) Electrostatic: The electrostatic energy harvesters use the relative motion between the plates of the variable capacitors to generate the electric current. In this method, the mechanical motion is used to cause the distance change between the electrodes of the capacitor. Hence resulting in voltage variation, thus, generating electric current in the connected circuit. Electrostatic systems offer high output voltages, adjustable coupling coefficients, low capacitances and cost effectiveness [260]. On the down side, these devices are relatively small and needs handling of micrometer dimensions.

3) Electromagnetic: The electromagnetic energy harvesting is one of the most widely used power generation mechanism, commonly used on the larger scales where the relative motion in electromagnets and the windings induces electric current in the windings. The synchronous generators, induction generators, permanent magnet generators are some of the examples 
TABLE XII: Primary Battery sources and Energy harvesting techniques in IWSNs [16], [32], [35], [270], [271], [272], [273]

\begin{tabular}{|c|c|c|c|c|}
\hline Sr. & Energy Sources & & Possible Variations & Power \\
\hline 1. & \multicolumn{4}{|c|}{ Primary Batteries } \\
\hline & & a) & Alkaline AA (E91) [274] & $1.5 \mathrm{~V}$, upto 3080mAh@40C \\
\hline & & b) & Lithium AA (L91) [275] & 1.5V, upto 3400mAh@40C \\
\hline & & c) & $\begin{array}{l}\text { Lithium-Manganese Dioxide } \\
\text { (Li/MnO2) (LA522) [276] }\end{array}$ & $9 \mathrm{~V}$, upto $850 \mathrm{mAh}$ \\
\hline \multicolumn{5}{|c|}{\begin{tabular}{l|l}
2. & Super Capacitors \\
\end{tabular}} \\
\hline & & a) & Maxwell BCAP0350 & $\begin{array}{l}2.5 \mathrm{~V}, 350 \mathrm{~F}, 5.73 \mathrm{Wh} / \mathrm{L} \text { with } \\
500,000 \text { life cycles [35] }\end{array}$ \\
\hline & & b) & Maxwell PC10 & $\begin{array}{l}2.5 \mathrm{~V}, 10 \mathrm{~F}, 1.4 \mathrm{Wh} / \mathrm{kg} \text { with } \\
500,000 \text { life cycles [35] }\end{array}$ \\
\hline & & c) & Green-cap EDLC(DB) & $\begin{array}{l}2.7 \mathrm{~V}, \quad 50 \mathrm{~F}, \quad 4.4 \mathrm{Wh} / \mathrm{L} \text { with } \\
\text { over } 100,000 \text { life cycles [35] }\end{array}$ \\
\hline & & d) & NEC TokinFT0H105Z & 5V, 1F, 1000 life cycles [35] \\
\hline \multirow[t]{4}{*}{3.} & \multicolumn{4}{|c|}{ Rechargeable Batteries } \\
\hline & & a) & NiMH & $\begin{array}{l}\text { 1.2V, upto 2500mAh@40C } \\
\text { [35] }\end{array}$ \\
\hline & & b) & Li-ion & $\begin{array}{l}\text { 3.7V, upto 730mAh@40C } \\
\text { [35] }\end{array}$ \\
\hline & & c) & Li-polymer & $\begin{array}{l}\text { 3.7V, upto 930mAh@40C } \\
\text { [35] }\end{array}$ \\
\hline \multirow[t]{2}{*}{4.} & \multicolumn{4}{|c|}{ Energy Harvesting Techniques } \\
\hline & Light & a) & $\begin{array}{l}\text { Indoor } \\
\text { Outdoor }\end{array}$ & $\begin{array}{l}10 \text { to } 100 \mathrm{~W} / \mathrm{cm} 2[16],[32], \\
{[46]} \\
0.15 \text { to } 15 \mathrm{~mW} / \mathrm{cm} 2 \quad[32], \\
{[46]}\end{array}$ \\
\hline & Temperature & - & - & $15 \mathrm{~W}$ to $60 \mathrm{~mW} / \mathrm{cm} 2[32]$ \\
\hline & Vibration & $\begin{array}{l}\text { a) } \\
\text { b) } \\
\text { c) }\end{array}$ & $\begin{array}{l}\text { Electrostatic } \\
\text { Electromagnetic } \\
\text { Piezoelectric [16] }\end{array}$ & $\begin{array}{l}50 \text { to } 100 \mathrm{~W} / \mathrm{cm} 3[32],[47] \\
0.2 \mathrm{~W} \text { to } 1 \mathrm{~mW} / \mathrm{cm} 3[32], \\
{[46],[47]} \\
10 \text { to } 100 \mathrm{~W} / \mathrm{cm} 3\end{array}$ \\
\hline & Wind/Airflow & - & - & $\begin{array}{l}0.1 \text { to } 100 \mathrm{~mW} / \mathrm{cm} 3 \text { (wind } \\
\text { speed } 2 \mathrm{~m} / \mathrm{s} \text { to } 9 \mathrm{~m} / \mathrm{s} \text { ) [47] }\end{array}$ \\
\hline & Thermal & - & - & 15 to $60 \mathrm{~W} / \mathrm{cm} 3[16]$ \\
\hline & $\begin{array}{l}\text { Nuclear diode } \\
\text { junction }\end{array}$ & - & - & $500 \mathrm{n}$ to $\mathrm{W} / \mathrm{cm} 3$ [268] \\
\hline
\end{tabular}

where electromagnetic induction is used for electrical power generation. In electromagnetic induction, the rotational motion achieved from wind, hydropower, gas and petrol engine is converted in the electric power. However, these structures are relatively large and are used to produce electrical energy in hundreds of megawatts. For IWSNs, the electromagnetic energy harvesting has great significance as in industries magnetic flux, rotational motion and magnetic fields are found in abundance, which can easily be transformed in small scale selfsustaining electromagnetic energy sources. Furthermore, the electromagnetic energy harvesting sources offer high output currents, robustness, and long lifetime. However, due the small size of these harvesters the energy efficiency is relatively low [260].

In general, vibration/motion energy produced from different uncontrollable/partially controllable processes, (wind, liquid flow, stress, strain, vibrate etc.) can result in diverse energy resources [264], [265]. The graphical representation of vibration energy harvesting is presented in Fig. 13, whereas the details of energy harvesting potential of these harvesters are presented in Table XII.
4) Summary and insights: Industrial environments are filled with the vibrations generated by a wide variety of operations including rotation, drilling, pressure, kinetic energy and biomotion. The use of energy harvesting techniques to scavenge energy from these sources is a common practice. Depending on the application and the node energy requirements, a decision can be made on the possible energy harvesting scheme.

The selection of the viable energy harvesting scheme depends on its suitability in a given application. For instance, evaluation of rotational speed, magnetic flux and generated heat in motors and generators require precise sensory feedback. For such cases, sensor nodes are usually placed on the rotor for accurate measurements. Since the sensor node is going through the rotational motion, hence the use of electromagnetic energy harvesters is more suitable. The electromagnetic energy harvesting nodes produce notable energy and can be used in a wide variety of applications. However,the use of permanent magnets in the electromagnetic energy harvesters lose magnetic properties over time and can cause reduction magnetic flux in turn reducing energy production capabilities with the passage of time. 
In the pressurized environments, use of piezoelectric energy harvesting appears to be a more viable option. The piezoelectric harvesting nodes although harvest plenty of power yet the material used in piezoelectric energy harvesters is relatively expensive.

Since the vibrational energy harvesting nodes are capable of fulfilling required energy needs, there are no limitations on applications and communication of vibration based energy harvesting nodes if a suitable vibrational energy harvesting source is available.

\section{Hybrid Energy Resources}

In order to improve the performance of the energy harvesting sources, primarily relying on single energy source, hybrid schemes are implemented. In certain cases, the certain energy sources are intrinsically linked and can be efficiently utilized. The use of solar PVs along with thermal power harvesting offers one such scenario where the photons as well as the heat of the sun is transformed in to electrical energy. It results in the improved efficiency per unit area. To employ multi-source harvesting, multiple harvesting modules are integrated in a system [35]. A hybrid system using maximum power point solar PVs and wind is presented in [266]. The design of the hybrid energy harvesters is presented in [267]. Some other example of hybrid energy harvesters is presented in [264].

The nuclear diode junction battery with long lasting ability can also serve as an alternate. The battery offers long life but relatively low power [268], [269].

Table XII lists some of the energy harvesting techniques used and their expected potential contribution along with conventional primary battery specifications, supercapacitor details and rechargeable batteries. All these techniques add additional lifetime to IWSNs fulfilling the basic requirements of the extended lifetime. Apart from the energy harvesters, a suitable electronic circuit with regulators, DC-DC converters and filtering is needed to feed the rechargeable batteries. Though in some cases battery-less operation is also opted for IWSNs, where the energy-harvester serves as the primary source of energy yet it limits the coverage of the sensor nodes.

1) Summary and insights: Use of the hybrid energy harvesting nodes is relatively rare in industrial environments. One of the primary reasons is the additional cost factor added to an individual node affecting the cost of the network significantly, especially in case of larger networks. However, in some cases where more frequent communications are required from the wireless sensor nodes deployed in remote or inaccessible area, hybrid schemes may be adopted. In the literature, some of the hybrid schemes are demonstrated over the years including thermal-PV, PV-wind, biochemicalbiomechanical [35], [46].

The use of the primary batteries, rechargeable batteries and supercapacitors can be found in almost all the applications in IWSNs due to the non-suitability of battery less nodes (green nodes) for industrial automation and process control. Due to the unique attributes of each of these battery sources, the preference of the individual battery type can vary depending on the system requirements. The rechargeable batteries are often used in combination with the energy harvesting techniques to offer longer network lifetime. The notable capacity of rechargeable batteries allows the use of less predictable energy harvesting techniques like solar so that the nodes can survive few days without recharge. However, one of the possible problems of the rechargeable batteries is the effect on battery lifetime with frequent recharges.

Super capacitors on the other hand, offer much higher recharge cycles without significantly wearing out. However, lower energy capacity limits the use of supercapacitors only in combination with the predictable energy harvesting techniques. The predictable attributes of the energy harvesting schemes ensure a constant source of energy hence allowing the nodes to mostly operate based on the energy harvested. However, to assist in the occasional variations in the energy harvested and anomalies in harvesting systems are addressed with use of supercapacitors. More frequent energy harvesting schemes used in combination with supercapacitors include vibrations and indoor solar harvesters.

The permanent batteries are used in non-accessible and remote wireless networks. The use of under-water sensor nodes in dams for evaluation of water $\mathrm{pH}$, impurities sediments water flow-in dissolved oxygen and temperature sensors serve as one such cases where the node lifetime lasts until the battery dies out. Furthermore, in some applications where the energy harvesting is not an option the permanent batteries are also used. In such cases the nodes are easily accessible and a regular battery replacement cycles are scheduled to run the processes smoothly.

\section{Good Practices and Design Solutions in IWSNS}

In IWSNs, continuous research has provided much wanted improvements in past few years. It is because of the efforts of many individuals and some joint ventures that IWSNs have recently witnessed much wider acceptability in all sorts of industrial applications. Due to the broader scope of the potential applications of IWSNs, it is becoming difficult to cope with the rising challenges. In this section, to resolve prominent challenges in IWSNs, certain practices are listed. Wherever appropriate, existing research is discussed to overcome these challenges, otherwise new directions for possible solutions are explored.

\section{A. Dynamic Priority Scheduling}

The communication in Industrial processes is not usually limited to one particular class or industrial system. For instance, an industrial process can be communicating emergency traffic, regulatory control traffic, alerting traffic and monitoring traffic. In such processes, the multilevel prioritized communication offers a better chance at effective communication optimization. Some of the priority based communication protocols are listed in Section VIII-A(4) and main strengths 
and weaknesses of these protocols are highlighted. However, a commonly observed trend is the use of pre-defined priorities for each industrial traffic type. Doing so, limits the response of communication protocol only to predefined states, and hence, its suitability to handle dynamic processes. Since the priority of traffic in industrial networks can change on runtime, depending on variations in sensor readings, the use of predefined traffic priority may not be a suitable solution. Hence, the widely-proposed priority based MAC protocols for IWSNs are inefficient in scenarios where certain variation in the existing priority can occur. The examples of such cases include Alerting systems, where violation in certain critical thresholds, in runtime, may result in instability in plant's operation. Under such circumstances the alerting system's traffic priority should be increased due to its current critical nature. Similarly realtime variation in supervisory control feedback also changes the priority of the affiliated traffic and more regular feedback may be desired.

Therefore, a more effective way to deal with processes like these is by establishing dynamic priority, which could prioritize in real-time. The concept behind dynamic priority scheduling is to form adaptive and evidence based decision of which information is more critical and must be delivered on priority. Inclusion of dynamic priority scheduling meets the challenges of time constraint and information priority scheduling by efficiently sacrificing the bandwidth of low priority data sources.

\section{B. Variable Sampling Rates and Heterogeneous Sensing}

The information generated in a plant is usually of critical nature but its significance rises even more when it violates critical thresholds. At such instances, it is imperative to observe status of such information more frequently. To do so, the design cannot rely on the static sampling rates rather it must adopt with the changing statistics of the information from different sections of the plant. Hence, the incorporation of variable sampling based on critical nature of information is imperative for better industrial solution. In literature, no significant contributions can be found in this aspect. However, the use of variable sampling rates, to compensate network loads and to trade-off between the frequently desired and less frequently desired information, can significantly improve the reliability of the network communication by effectively managing bandwidth fluctuations and resource constraints.

Heterogeneous sensing, is usually referred in similar meanings as variable sampling. However, to establish a difference between the two, the heterogeneous sensing is used in sense of quantization information and number of bits assigned to the sensed information. As in IWSNs, it is of paramount importance that the information from different sources is dealt according to the nature of information, not only in sense of priority but also in terms of sampling rates and affordable quantization error of the sensed information. Therefore, heterogeneous sensing, though rarely used, can make a significant difference in reducing the load of stressed out networks by down scaling the number of bits used to represent an analog sensor value. The decision on when to reduce the precision of the sensed information is question of how significant the sensed information is and will it affect the process outcome or not.

This compels the researchers to optimize the information preferences and allocate the resources accordingly in order to get the desired results. Thus, merging heterogeneous signals and the allocation of non-uniform resources must primarily be considered in design.

\section{Data Fusion and Localized Processing}

Data fusion is an important aspect of IWSNs and can be greatly beneficial in harsh industrial applications. Localized processing with efficient data fusion can greatly help in transforming massive data into compressed critical information. The localized processing also reduces the time delays between reading sensor values to controlling the actuators. If efficiently implemented, this process can help in eliminating some crucial constraints in IWSNs including data redundancy, time constraint, network lifetime and even BER.

\section{Efficient Information Scheduling}

Information scheduling plays a very important role in heterogeneous industrial networks. In a system with different processes integrated together, the sensed data originating from different sources may have very diverse time deadlines, sampling rates, priority levels and failure consequences. Some of the existing scheduling schemes presented over the years are discussed in Section VIII-A(2). Apart from this, the IEEE802.15.4e discussed in Section VI-B(2) also offers a base platform which can be further enhanced to optimize scheduling. However, there is much more potential for improvement where an efficient scheduling of information can resolve many intrinsic problems of Synchronization, Sleep Scheduling, Contention free medium access, Coexistence, reliability, interference and BER. Thus, where a good scheduler design is of principal importance in TDMA based industrial communication standard, a poor scheduler may lead to violation of time constraints in harsh industrial environments.

\section{E. Long Lifetime Assurance}

A long network lifetime has much importance in IWSNs especially in automation and process control applications. With the improving technologies, a great extension in the lifetime of the industrial equipment has been witnessed. To cope with this, energy harvesting techniques must be considered to offer extended lifetime of IWSN nodes, in comparison to that of the industry equipment. A detailed discussion is being provided on various energy harvesting schemes, capacity of the batteries and supercapacitors in Section X, yet, the practical evaluation of these schemes in industrial environments can lead to a more accurate time and capacity information of various schemes discussed. Another interesting evaluation would be to link the energy harvesting schemes with the power consumption of various motes available in the market along with the use of low latency, reliable and energy efficient algorithms [275], [276]. A thorough research in this domain may lead to a better estimate of the lifetime expectancy of IWSN motes with different energy harvesting techniques. 


\section{F. Data Reliability, Time Synchronization and Retransmission Scheduling}

Reliable data communication has great significance in IWSNs and accuracy of each transmission is very important. It is for the same reasons, retransmission is considered to be very important to achieve the desired levels of QoS. In this aspect, IEEE802.15.4e can serve as a good example as it offers interference free channel access. It also implements Time Synchronized Channel Hopping (TSCH) for better reliability. However, the desired Packet Reception Rate (PRR) could be a challenge due to some significant interference sources, intrinsic to the industrial environments. This is where the desired QoS is compromised. However, to ensure a particular QoS at all times for certain communication types in IWSNs, the retransmission scheduling can be used. The shared slots provided in IEEE802.15.4e can be used for this purpose, but the default retransmission uses a CSMA/CA based channel access during the shared slots which makes the reliability and timely delivery of already delayed information questionable. In this aspect, very limited research can be found. The available research includes works in [10] and [55]. In [10], authors try to improve the retransmission efficiency in TDMA based multihop IWSNs, using efficiently scheduling the shared slots. In [55], a priority mechanism is defined which attempts to improve the QoS of high priority communication, by allowing it to retransmit the failed communications of high significance over the time slots assigned to lower priority communications, hence offering better reliability. Further improvements are also expected in this domain however it requires more investigation and research for optimized solutions.

\section{G. Application Specific and Resource Efficient Design}

Careful observation of the parameters in a plant can give insight of the suitable solutions for the selected applications. A prime solution in the field of IWSNs that can perfectly fit every application is a myth. Therefore, one cannot deny the need for an application specific design in IWSNs [16]. Apart from this, if the wireless nodes are used to develop a generalized solution which can work in a wide variety of applications, it might need a lot of resources. For instance, if the nodes are developed to be compatible with Zigbee, WirelessHART and 6LowPAN standards at once, it will require multiple radios and secondary processing units. Keeping in view the maintenance, modifications, deployment packaging, servicing, and unit costs, the solutions becomes unfeasible. Therefore, most of the applications tend to offer compatibility with a single communication protocol where few special nodes can work as a gateway to interconnect with other technologies. An application specific design offers more promise of fulfilling the desired objectives with minimum resources and lowest cost possible. In Section VII-A, a detailed discussion on motes and radios available in the market can be considered to identify suitable motes for required applications. Furthermore, some other key elements need to be opted, based of application requirements are listed in Section IV-D. With all the basic objectives covered, one may look further to optimize efficiency of the design by incorporating transmission power control, sleep scheduling and energy harvesting for network lifetime improvement, distributed processing, linearization, effective memory mapping and code optimization for resource efficient design and modular approach, clustering, and hierarchical design for scalable solutions [277].

However, to propose a solution suitable for the application as well as to offer possible potential for future developments is a challenging task. Apart from this an industrial solution based on WSNs is expected to be efficient and scalable. The IWSNs not only have to adjust with the existing field-bus and Ethernet legacy solutions but also have to deal with the changing and ever expanding industrial structure. In such cases, modular approach and hierarchical based solution is recommended for the scalable architectures. Furthermore, it is recommended to efficiently utilize the resources from implementing efficient memory allocation algorithms to the sophisticated techniques to prolong the network lifetime.

\section{Future Research Directions}

IWSNs offer a suitable alternate for the industrial monitoring, automation and process control and it is expected that the future investments in IWSNs will further increase. Apart from many appealing benefits, primary cause of market boost in IWSNs is the significant price differences in wireless and wired networks. However, there are still some concerns from control and automation industry and experts of the field, highlighting the QoS relate problems of the wireless technology. It is, therefore, foreseen that certain critical areas will receive significant amounts of attention in the future to cope with the QoS offered by the typical wired networks.

It is therefore predictable that the future research will try to compensate the lag in IWSNs to offer reliable communication in industrial applications with comparable quality metric values (BER, RSSI, data rate etc.) to that of the wired networks. Moreover, to meet the high data rate requirements in higher hierarchy of IWSNs, other technologies like Visible Light Communication (VLC) [278], [279], Cognitive Radios (CR) [280], [281] and IoT [282] will play an important role. Main research streams in IWSNs to cope with the industrial demands are listed as under.

\section{A. Deterministic Network Formation}

Since IWSNs are not yet considered as a mature technology in industrial automation and control, ensuring deterministic behavior in IWSNs will be receiving great significance in the next few years. It is very important to offer deterministic behavior of IWSNs to assure proper working in harsh environments and industrial processes. Despite a lot of work in Physical, MAC and Network layer optimizations, still most of it needs to converge on a single platform to offer consolidated solution in the formation of deterministic networks where one could predict the operation with certainty. Moreover, the reliability assurance in IWSNs will always be compared to the existing wired solutions irrespective of the cost difference. It is, therefore, needed to conduct dedicated research in this sensitive area. Though with the inclusion of TDMA, channel access reliability is certainly improved, yet the added delay 
needs to be carefully handled without sacrificing the deterministic network architecture. Certain reliability, real-time delivery, scheduling and priority optimization protocols [30], [36], [38], [41], [53], [54], [55], [62], [66], [97], [108], [109], [193], [207], [275], [276], [283], [284] (Section VI, VIII and IX provide sufficient information on the listed optimization protocols) are also defined. However, for most of the cases static network architectures were investigated while most of the practical applications demand dynamic and scalable network architecture for durable design.

\section{B. Durable and Long Life IWSNs}

The extension in the lifetime of industrial equipment has forced the need for the energy harvesting techniques in IWSNs. Moreover, some hard-to-access deployment points (turbines, internal machinery/generators sensors etc.) urge the extensive lifetime of the wireless sensor nodes. By far, literature lacks a thorough evaluation of energy harvesting techniques specifically targeting industrial environment, its energy generation potentials and presentation of application specific energy harvesting techniques for industrial environments. A thorough evaluation of potential of energy harvesting techniques with suitable blend of variety to cover the wide scope of industrial applications is very important. Such effort will encourage further investigation in this area to unleash the full potential of IWSNs in industries. Apart from this, research on hybrid energy sources is very limited and if properly investigated can unlock infinite potential for future IWSNs.

\section{Interoperability in IWSNs}

Interoperability and transformation of existing structure to wireless with minimal variation in the existing setup will greatly help the transformation. Moreover, the need for wireless solutions with more flexibility and ability to embed in the existing wired networks is missed and is strongly desirable for potential improvements. Although some industrial protocols offer interoperability in IEEE802.15.4 and TCP/IP operated devices, yet it only accounts for the Ethernet based wired networks. Also the need for translation from full duplex (wired setup) to half duplex (wireless setup) is much needed. Apart from these, many wireless technologies are predicted to collaborate in industrial automation and process control. Unfortunately, scarcity of the radio band would demand operation of various wireless technologies in overlapping spatiotemporal regions which demand expansion of IWSNs to other technologies to assist in future industrial automation and process control.

\section{Industrial IoT}

Industrial Internet of Things (IIoT) is a specific branch of IoT which addresses the communication and connectivity issues in industrial environments and offers suitable solutions. IIoT can improve interconnectivity, flexibility, scalability, time efficiency, cost effectiveness, security, productivity and operational efficiency in the industries. IoT can also serve as a platform to establish intelligent network of devices [285] which can interrelate data and processes to effectively establish feedback control systems for industrial automation. The research in use of IoT in industrial environments is increasing exponentially with IIoT emerging as a main contender in the field of wireless industrial automation [286]. Apart from the contributions of research community in improving the operability of IoT in the industries, many dedicated hardware modules and platforms are also introduced to support the IoT based developments in the industrial environments. A list of various practical modules optimized for IoT are discussed in Section VII-A.

The developments in IIoT aim at offering an accomplished technology capable of integrating heterogeneous sensors and systems. To maximize the operational efficiency and to offer extended benefits, use of multi-layer Service Oriented Architecture (SOA), with some variations, is proposed [287], [286]. Energy consumption, latency, extensibility, modularity, scalability, topology, throughput, security and safety are some of the key considerations shaping SOA [288]. For process control and automation applications, sensors and actuators need to interact with the environment more frequently and may have real-time communication and response requirements, hence an adaptive architecture is needed to dynamically interact with the environment. Furthermore, the architecture should also support the heterogeneous and decentralized operation of IoT. Therefore, SOA is considered an appropriate approach to achieve interoperability between heterogeneous devices in a multitude of way [286], [289], [290].

Some initiatives from the research community and standards organizations have facilitated the adaptation of IoT in industrial environments. 6LoWPAN is one such development which targeted, IPv6 implementation over Low-power WPAN, details of which can be found in Section VI-C(4). Details of some other developments and initiatives are listed as follows.

1) OpenWSN: OpenWSN is an open-source project which facilitates the implementation of IoT in time and reliability sensitive resource constrained networks. The free and opensource implementation of the IoT oriented protocol stack and development of debugging, support and integration tools in OpenWSN encourages rapid development in IoT centered low power mesh networks [291], [292]. OpenWSN implements standards based protocol stack for IEEE802.15.4e Time Synchronized Channel Hopping (TSCH) based resource constrained networks coupled with IoT standards like 6LowPAN (the IPv6 protocol adaptation layer to IEEE802.15.4 networks [293]), RPL (routing protocol for Low-Power Lossy Networks (LLN) [294]) and CoAP (a light-weight HTTP-alike application protocol [295]) to aid ultra-low power and reliable mesh networking in internet enabled WSN [291]. Use of IEEE802.15.4e TSCH MAC ensures suitability of OpenWSN for time and reliability constrained industrial networks.

The developments in OpenWSN can be categorized in two sub-projects, firmware and software. The firmware is written in standard C99 and GCC is used as a default compiler which enables compatibility of the firmware with AVR, ARM cortex and MSP processor architectures[292]. OpenVisualizer, the software sub-project, developed using Python, serves as an internet gateway and is responsible for IPv6 compression, 
network topology, routing, statistics and essential packet metrics. In OpenWSN the flexibility is added using command line options which allow the users to combine toolchains, kernals applications and board with single command line [292]. Furthermore, the continuous integration servers provide open IIoT service which allows end-users to build applications online.

2) 6lo WG: IPv6 over Networks of Resource-constrained Nodes (6lo) Working Group replaces 6LoWPAN WG to extend the IoT enabled and IPv6 supported protocol stack for wider range of radio technologies. It is an initiative by Internet Engineering Task Force (IETF) where 6 lo WG is intended to provide suitable support for IoT and IPv6 to resolve interconnectivity issues in primitive radio technologies. The primary objectives of 6lo is to facilitate IPv6 connectivity over resource constrained networks with following characteristics [296].

- Low power, processing and memory resources

- Strict upper bounds on state, code space and processing cycles

- Optimization of energy and network bandwidth usage

- Lack of some layer 2 services like complete device connectivity and broadcast/multicast

The developments in 6lo aim at several radio technologies where the main areas of interest include [297]

- Transmission of IPv6 packets over Digital Enhanced Cordless Telecommunications (DECT) Ultra Low Energy (ULE)

- Transmission of IPv6 packets over Master-Slave/TokenPassing (MS/TP) Networks

- Transmission of IPv6 packets over Near Field Communication (NFC)

- Transmission of IPv6 packets over BLUETOOTH(R) Low Energy (BLE)

- Transmission of IPv6 packets IPv6 Packets over DECT Ultra Low Energy

- Transmission of IPv6 packets IPv6 over Low-Power Wireless Personal Area Networks (6LoWPANs)

- Transmission of IPv6 over MS/TP Networks

- Transmission of IPv6 packets over ITU-T G.9959 Networks

\section{E. IWSNs and VLC}

In industrial applications, Ethernet based standards offer significant data rates in multiples of tens of megabits per second to handle high speed traffic. To handle large amounts of data in higher hierarchies of the IWSNs, the IEEE 802.15.4 based industrial standards may not be able to offer sufficient bandwidth and may need a different technology which could support high data rates. For such cases, VLC technology can serve as a suitable replacement to the existing high speed Ethernet [298], [299], [300]. Some other benefits of the technology include the non-existence of interference among VLC and radio frequency based IWSNs as well as relatively higher security compared to traditional technologies.

\section{F. Cognitive Sensor Networks for Industrial Applications}

IWSNs use Industrial, Scientific and Medical (ISM) band, a frequency spectrum utilized by many other technologies as well. The continuous increase in the use of wireless technologies in various applications are and will be soon leading to overlapping wireless spectrum access where the opportunistic spectrum access would be inevitable [280]. Examples of such cases can be seen in Wi-Fi, Zigbee, Bluetooth and Wi-MAX, where all of these use overlapping spectrum [301], [302], [303]. To avoid the interference under similar circumstances, use of cognitive sensor networks for opportunistic spectrum access may be the only solution. Moreover, CR also offer a scope of bandwidth extension and multichannel utilization by opportunistic tapping in unused spectrum which is otherwise not usable in traditional IWSNs [281], [304]. Cognitive Radio Sensor Networks (CRSNs) are suitable for handling nonlinearly distributed sensors for reliable data delivery QoS under unfavorable propagation conditions [305].

Industrial and factory automation, process control and distributed control systems are appealing more and more wireless technologies [306], [307]. Although with the use of TDMA based channel access the channel congestion and collisions are greatly reduced, but the scheme is not very successful in guaranteed channel access if multiple technologies are working in same vicinity. CR, under such circumstances can assist in optimizing communication reliability along with the provision of interoperability among different technologies. Apart from this, channel bonding in CRSNs should also be considered for improved reliability and delay parameters. A survey of channel bonding CRSN is presented in [308]. There are also many protocols which have been refined over the years for Cognitive radios and can be transformed for CRSNs. Cognitive radio protocols involving spectrum sensing, spectrum allocation and spectrum handoff can be optimized for sensor networks to offer better efficiency [309]. Apart from this, the cognitive radio power control mechanism offers extended network lifetime along with reduced interference [310]. Some of the existing architectures (Spectrum pooling, CORVUS, IEEE802.22, DIMSUMnet, DRiVE, OverDRive, Nautilus [311], [312]) can also be utilized to signify the benefits of cognitive sensor networks over traditional sensor networks.

All these benefits vouch for the cognitive sensor networks as a potential technology in industrial automation and process control. Opportunistic spectrum access in cognitive radios has been thoroughly evaluated and offers sufficient and well evaluated works in literature. All these developments can easily be translated in IWSNs to offer improved performance in industrial automation and process control applications by assuring superior communication reliability and collision avoidance. However, use of cognitive sensor networks in industrial automation and process control is still in infancy and need to be further explored for analysis of this potential technology in industrial applications. The security assurance in CRSN is also a main issue and still several challenges and open research issues are remaining [313]. Some of the work in this particular aspect, as discussed earlier, [280], 
[306], [307], [314], [315], [316], [317], [318], can serve as a baseline for developing the next generation CRSNs for monitoring applications, emergency responses, process control and automation.

\section{CONCLUSION}

IWSNs have shown great potential in improving the processes and cost efficiency in industrial applications. However, many challenging factors still limit the performance of IWSNs in extreme industrial environments. At present, the potential of IWSNs is limited by many factors. It is therefore necessary to target the key limiting areas to exploit the full potential of IWSNs. The research in past few years has pushed IWSNs to the verge of wide acceptability in industrial automation and process control. Yet there is a need of further improvement in the areas including, reliability, real-time data delivery, lifetime enhancement, security, localized processing, efficient route selection, scalability, modular design, information aggregation, data compression, interoperability and deterministic network design. Any improvement in these areas would promote IWSNs towards industrial applications, which could affect the process control and automation industry on broader scales by providing low cost information from the entire plant, adding to efficiency of the processes and introducing significant cost reduction in the information collection and process control.

IWSNs introduce efficient means to communicate information within the industrial processes and have foreseeable future in industrial monitoring, process control and automation. But applications of IWSNs are not limited to information communication. Rather, the technology enables IWSNs to embed intelligence in the industrial processes. With the ability to offer localized processing and decision making, each node, individually, and each network, collectively, can serve as an evolutionary technology for industries. Still IWSNs are far from reaching the stated potential and need continuous improvements as well as collaborative progress to reach potentially promising future.

In this review, an effort is made to present the IWSNs from a different perspective. The paper offers a thorough survey on IWSNs, and attempts to encompass sufficient and significant work in this field including the mote designs, radios, protocols and standards, research contributions, developments in energy harvesting and batteries, main industry contributors and primary industry requirements. The paper also evaluates the gaps in technology and the industrial requirements, which could challenge the performance of IWSNs, and offers possible way out to exploit full potential of IWSNs. It also gives an insight of future technologies and the trends that could be observed in coexistence with IWSNs in future in automation and process industries.

\section{ACKNOWLEDGMENT}

Author would like to thank the European Erasmus Mundus cLINK programme and Northumbria University for funding the research. Author would also like to thank Northumbria University, UK and Capital University of Science and Technology, Pakistan for facilitating the collaborative research and provision of research facilities.

\section{REFERENCES}

[1] C. Ying-Chyi, L. Ching-hua, and C. Pao-Long, "Using theory of constraints to find the problem about high level inventory in the aerospace industry," in Technology Management for Global Economic Growth (PICMET), 2010 Proceedings of PICMET '10:, 2010, pp. 1-10.

[2] A. Z. Xu. (2014, 2020: Future automation. Available: http://www.controleng.com/single-article/2020-future-automation/c33ed4 679973dcb1ed2f53411520088d.html

[3] T. M. Chiwewe and G. P. Hancke, "A Distributed Topology Control Technique for Low Interference and Energy Efficiency in Wireless Sensor Networks," Industrial Informatics, IEEE Transactions on, vol. 8, pp. 1119, 2012.

[4] M. Magno, D. Boyle, D. Brunelli, B. O’Flynn, E. Popovici, and L. Benini, "Extended Wireless Monitoring Through Intelligent Hybrid Energy Supply," Industrial Electronics, IEEE Transactions on, vol. 61, pp. 18711881, 2014.

[5] L. Palopoli, R. Passerone, and T. Rizano, "Scalable Offline Optimization of Industrial Wireless Sensor Networks," Industrial Informatics, IEEE Transactions on, vol. 7, pp. 328-339, 2011.

[6] S. Sooyeon, K. Taekyoung, J. Gil-Yong, P. Youngman, and H. Rhy, "An Experimental Study of Hierarchical Intrusion Detection for Wireless Industrial Sensor Networks," Industrial Informatics, IEEE Transactions on, vol. 6, pp. 744-757, 2010.

[7] O. Kreibich, J. Neuzil, and R. Smid, "Quality-Based Multiple-Sensor Fusion in an Industrial Wireless Sensor Network for MCM," Industrial Electronics, IEEE Transactions on, vol. 61, pp. 4903-4911, 2014.

[8] S. X. Ding, Z. Ping, Y. Shen, and E. L. Ding, "An Integrated Design Framework of Fault-Tolerant Wireless Networked Control Systems for Industrial Automatic Control Applications," Industrial Informatics, IEEE Transactions on, vol. 9, pp. 462-471, 2013.

[9] C. Min, "Reconfiguration of Sustainable Thermoelectric Generation Using Wireless Sensor Network," Industrial Electronics, IEEE Transactions on, vol. 61, pp. 2776-2783, 2014.

[10] Y. Dong, X. Youzhi, W. Hongchao, Z. Tao, Z. Hao, Z. Hongke, et al., "Assignment of Segmented Slots Enabling Reliable Real-Time Transmission in Industrial Wireless Sensor Networks," Industrial Electronics, IEEE Transactions on, vol. 62, pp. 3966-3977, 2015.

[11] B. Carballido Villaverde, S. Rea, and D. Pesch, "InRout A QoS aware route selection algorithm for industrial wireless sensor networks," Ad Hoc Networks, vol. 10, pp. 458-478, 2012.

[12] S. Misra, S. Goswami, C. Taneja, A. Mukherjee, and M. S. Obaidat, "A PKI Adapted Model for Secure Information Dissemination in Industrial Control and Automation 6LoWPANs," Access, IEEE, vol. 3, pp. 875-889, 2015.

[13] T. Blevins, C. Deji, H. Song, M. Nixon, and W. Wojsznis, "Process Control over Real-Time Wireless Sensor and Actuator Networks," in High Performance Computing and Communications (HPCC), 2015 IEEE 7th International Symposium on Cyberspace Safety and Security (CSS), 2015, pp. 1186-1191.

[14] J. Cecilio, P. Martins, J. Costa, and P. Furtado, "State machine modelbased middleware for control and processing in industrial wireless sensor and actuator networks," in Industrial Informatics (INDIN), 10th IEEE International Conference on, 2012, pp. 1142-1147.

[15] Y. Zhiyuan, S. Yulei, and N. H. El-Farra, "Resource-aware scheduled control of distributed process systems over wireless sensor networks," in American Control Conference (ACC), 2010, pp. 4121-4126.

[16] V. C. Gungor and G. P. Hancke, "Industrial Wireless Sensor Networks: Challenges, Design Principles, and Technical Approaches," Industrial Electronics, IEEE Transactions on, vol. 56, pp. 4258-4265, 2009.

[17] J. Akerberg, M. Gidlund, and M. Bjorkman, "Future research challenges in wireless sensor and actuator networks targeting industrial automation," in Industrial Informatics (INDIN), 9th IEEE International Conference on, 2011, pp. 410-415.

[18] F. Dobslaw, M. Gidlund, and Z. Tingting, "Challenges for the use of data aggregation in industrial Wireless Sensor Networks," in Automation Science and Engineering (CASE), IEEE International Conference on, 2015, pp. 138-144.

[19] A. A. Kumar Somappa, K. vsthus, and L. M. Kristensen, "An Industrial Perspective on Wireless Sensor Networks 2014; A Survey of Requirements, Protocols, and Challenges," Communications Surveys \& Tutorials, IEEE, vol. 16, pp. 1391-1412, 2014.

[20] "IEEE Standard for Local and metropolitan area networks-Part 15.4: Low-Rate Wireless Personal Area Networks (LR-WPANs) Amendment 1: MAC sublayer,' IEEE Std 802.15.4e-2012 (Amendment to IEEE Std 802.15.4-2011), pp. 1-225, 2012. 
[21] "IEEE Standard for Information technology- Local and metropolitan area networks- Specific requirements- Part 15.4: Wireless Medium Access Control (MAC) and Physical Layer (PHY) Specifications for Low Rate Wireless Personal Area Networks (WPANs)," IEEE Std 802.15.42006 (Revision of IEEE Std 802.15.4-2003), pp. 1-320, 2006.

[22] "IEEE Standard for Information Technology - Telecommunications and Information Exchange Between Systems - Local and Metropolitan Area Networks Specific Requirements Part 15.4: Wireless Medium Access Control (MAC) and Physical Layer (PHY) Specifications for Low-Rate Wireless Personal Area Networks (LR-WPANs)," IEEE Std 802.15.42003, pp. 1-670, 2003.

[23] "IEEE Standard for Local and metropolitan area networks-Part 15.4: Low-Rate Wireless Personal Area Networks (LR-WPANs)," IEEE Std 802.15.4-2011 (Revision of IEEE Std 802.15.4-2006), pp. 1-314, 2011.

[24] Z. Tang, Z. Mei, P. Zeng, and H. Wang, "Industrial wireless communication protocol WIA-PA and its interoperation with Foundation Fieldbus," in Computer Design and Applications (ICCDA), 2010 International Conference on, 2010, pp. V4-370-V4-374.

[25] K. Al Agha, M. H. Bertin, T. Dang, A. Guitton, P. Minet, T. Val, et al., "Which wireless technology for industrial wireless sensor networks? The development of OCARI technology," Industrial Electronics, IEEE Transactions on, vol. 56, pp. 4266-4278, 2009.

[26] WirelessHART Overview, H. C. Foundation, (2016). Available: http://en.hartcomm.org/hcp/tech/wihart/wireless_overview.html

[27] 6loWPAN Active Drafts, (2016). Available: https://datatracker.ietf.org/ $\mathrm{doc} / \mathrm{search} /$ ?name $=61 \mathrm{loWPAN} \&$ activeDrafts $=$ on $\& \mathrm{rfcs}=$ on

[28] Utility Industry, The ZigBee Alliance, (2016). Available: http://www.zigbee.org/what-is-zigbee/utility-industry/

[29] ISA-100 Wireless Compliance Institute, ISA-100 Wireless Compliance Institute-Official Site of ISA100 Wireless Standard, (2016). Available: http://www.isa100wci.org/

[30] T. Zheng, M. Gidlund, and J. Akerberg, "WirArb: A New MAC Protocol for Time Critical Industrial Wireless Sensor Network Applications," Sensors Journal, IEEE, vol. PP, pp. 1-1, 2015.

[31] B. C. Villaverde, S. Rea, and D. Pesch, "InRoutA QoS aware route selection algorithm for industrial wireless sensor networks," Ad Hoc Networks, vol. 10, pp. 458-478, 2012.

[32] C. Viehweger, T. Keutel, and O. Kanoun, "Energy harvesting for wireless sensor nodes in factory environments," in Systems, Signals \& Devices (SSD), 11th International Multi-Conference on, 2014, pp. 1-4.

[33] Industrial Wireless Sensor Networks Market worth $\$ 944.92$ Million by 2020, (December 2015). Available: http://www.marketsandmarkets.com/ PressReleases/wireless-sensor-network.asp

[34] Industrial Wireless Sensor Networks Market worth $\$ 3.795$ Billion by 2017, (December 2015). Available: https://docs.zoho.com/printpdf.do?doc Id=yjvcr1e55cfc490b7464194dea2a 78611 eb68

[35] F. Akhtar and M. H. Rehmani, "Energy replenishment using renewable and traditional energy resources for sustainable wireless sensor networks: A review," Renewable and Sustainable Energy Reviews, vol. 45, pp. 769784, 2015.

[36] H. Pei, X. Li, S. Soltani, M. W. Mutka, and X. Ning, "The Evolution of MAC Protocols in Wireless Sensor Networks: A Survey," Communications Surveys \& Tutorials, IEEE, vol. 15, pp. 101-120, 2013.

[37] P. Baronti, P. Pillai, V. W. Chook, S. Chessa, A. Gotta, and Y. F. Hu, "Wireless sensor networks: A survey on the state of the art and the 802.15. 4 and ZigBee standards," Computer communications, vol. 30, pp. 1655 1695,2007

[38] P. Suriyachai, U. Roedig, and A. Scott, "A survey of MAC protocols for mission-critical applications in wireless sensor networks," Communications Surveys \& Tutorials, IEEE, vol. 14, pp. 240-264, 2012.

[39] S. Sudevalayam and P. Kulkarni, "Energy Harvesting Sensor Nodes: Survey and Implications," Communications Surveys \& Tutorials, IEEE, vol. 13, pp. 443-461, 2011.

[40] G. Zhao, "Wireless sensor networks for industrial process monitoring and control: A survey," Network Protocols and Algorithms, vol. 3, pp. 46-63, 2011.

[41] M. Doudou, D. Djenouri, and N. Badache, "Survey on Latency Issues of Asynchronous MAC Protocols in Delay-Sensitive Wireless Sensor Networks," Communications Surveys \& Tutorials, IEEE, vol. 15, pp. 528$550,2013$.

[42] D. Christin, P. S. Mogre, and M. Hollick, "Survey on wireless sensor network technologies for industrial automation: The security and quality of service perspectives," Future Internet, vol. 2, pp. 96-125, 2010.

[43] A. Flammini, P. Ferrari, D. Marioli, E. Sisinni, and A. Taroni, "Wired and wireless sensor networks for industrial applications," Microelectronics Journal, vol. 40, pp. 1322-1336, 2009.
[44] I. Demirkol, C. Ersoy, and F. Alagoz, "MAC protocols for wireless sensor networks: a survey," IEEE Communications Magazine, vol. 44, pp. 115-121, 2006.

[45] A. Bachir, M. Dohler, T. Watteyne, and K. K. Leung, "MAC essentials for wireless sensor networks," Communications Surveys \& Tutorials, IEEE, vol. 12, pp. 222-248, 2010.

[46] R. V. Prasad, S. Devasenapathy, V. S. Rao, and J. Vazifehdan, "Reincarnation in the ambiance: Devices and networks with energy harvesting," IEEE Communications Surveys \& Tutorials, vol. 16, pp. 195-213, 2014

[47] M. L. Ku, W. Li, Y. Chen, and K. R. Liu, "Advances in Energy Harvesting Communications: Past, Present, and Future Challenges," IEEE Communications Surveys \& Tutorials, vol. 18, pp. 1384-1412, 2015.

[48] X. Lu, P. Wang, D. Niyato, D. I. Kim, and Z. Han, "Wireless networks with RF energy harvesting: A contemporary survey," IEEE Communications Surveys \& Tutorials, vol. 17, pp. 757-789, 2015.

[49] C. R. Valenta and G. D. Durgin, "Harvesting wireless power: Survey of energy-harvester conversion efficiency in far-field, wireless power transfer systems," IEEE Microwave Magazine, vol. 15, pp. 108-120, 2014.

[50] K. Islam, S. Weiming, and W. Xianbin, "Wireless Sensor Network Reliability and Security in Factory Automation: A Survey," Systems, Man, and Cybernetics, Part C: Applications and Reviews, IEEE Transactions on, vol. 42, pp. 1243-1256, 2012.

[51] P. Zand, S. Chatterjea, K. Das, and P. Havinga, "Wireless industrial monitoring and control networks: The journey so far and the road ahead," Journal of sensor and actuator networks, vol. 1, pp. 123-152, 2012.

[52] L. Zheng, "Industrial wireless sensor networks and standardizations: The trend of wireless sensor networks for process autometion," in Proceedings of SICE Annual Conference, 2010.

[53] L. F. M. de Moraes and R. S. Silva, "Analysis of multichannel wireless networks With priority-based polling MAC protocols," in Wireless Days (WD), IFIP, 2014, pp. 1-6.

[54] Z. Meng, L. Junru, L. Wei, and Y. Haibin, "A priority-aware frequency domain polling MAC protocol for OFDMA-based networks in cyberphysical systems," Automatica Sinica, IEEE/CAA Journal of, vol. 2, pp. 412-421, 2015.

[55] S. Wei, Z. Tingting, F. Barac, and M. Gidlund, "PriorityMAC: A Priority-Enhanced MAC Protocol for Critical Traffic in Industrial Wireless Sensor and Actuator Networks," Industrial Informatics, IEEE Transactions on, vol. 10, pp. 824-835, 2014.

[56] H. Liqun and N. W. Bergmann, "System requirements for industrial wireless sensor networks," in Emerging Technologies and Factory Automation (ETFA), IEEE Conference on, 2010, pp. 1-8.

[57] P. Neumann, "Communication in industrial automationWhat is going on?," Control Engineering Practice, vol. 15, pp. 1332-1347, 2007.

[58] J. Xia, C. Zhang, R. Bai, and L. Xue, "Real-time and reliability analysis of time-triggered CAN-bus," Chinese Journal of Aeronautics, vol. 26, pp. 171-178, 2013.

[59] Falco, Joseph, James Gilsinn, and Keith Stouffer. "IT security for industrial control systems: Requirements specification and performance testing." NDIA Homeland Security Symposium \& Exhibition. 2004.

[60] Stouffer, Keith, Joe Falco, and Karen Scarfone. "Guide to industrial control systems (ICS) security.” NIST special publication 800.82 (2011): 16-16.

[61] I. F. Akyildiz and M. C. Vuran, Wireless sensor networks vol. 4: John Wiley \& Sons, 2010.

[62] D. Kumar, T. C. Aseri, and R. Patel, "Multi-hop communication routing (MCR) protocol for heterogeneous wireless sensor networks," International Journal of Information Technology, Communications and Convergence, vol. 1, pp. 130-145, 2011.

[63] S. K. Vuppala, A. Ghosh, K. A. Patil, and K. Padmanabh, "A scalable WSN based data center monitoring solution with probabilistic event prediction," in Advanced Information Networking and Applications (AINA), IEEE 26th International Conference on, 2012, pp. 446-453.

[64] A. Salhieh, J. Weinmann, M. Kochhal, and L. Schwiebert, "Power efficient topologies for wireless sensor networks," Parallel Processing, International Conference on, 2001, pp. 156-163.

[65] J. Hwang, C. Shin, and H. Yoe, "Study on an agricultural environment monitoring server system using wireless sensor networks," Sensors, vol. 10, pp. 11189-11211, 2010.

[66] Q. Pham Tran Anh and K. Dong-Sung, "Enhancing Real-Time Delivery of Gradient Routing for Industrial Wireless Sensor Networks," Industrial Informatics, IEEE Transactions on, vol. 8, pp. 61-68, 2012.

[67] Pottner, Wolf-Bastian, et al. "Constructing schedules for time-critical data delivery in wireless sensor networks." ACM Transactions on Sensor Networks (TOSN) 10.3 (2014) 
[68] G. P. Hancke and V. C. Gungor, "Guest editorial special section on industrial wireless sensor networks," Industrial Informatics, IEEE Transactions on, vol. 10, pp. 762-765, 2014

[69] M. Erol-Kantarci and H. T. Mouftah, "Wireless multimedia sensor and actor networks for the next generation power grid," Ad Hoc Networks, vol. 9, pp. 542-551, 2011.

[70] Applications of Industrial Plant 3D Laser Scanning and Modeling. (2015) Available: http://www.geomapres.com/industry/engineeringverticals/

[71] J. Slipp, M. Changning, N. Polu, J. Nicholson, M. Murillo, and S. Hussain, "WINTeR: Architecture and Applications of a Wireless Industrial Sensor Network Testbed for Radio-Harsh Environments," in Communication Networks and Services 6th Annual Research Conference, CNSR , 2008, pp. 422-431.

[72] M. Wehner, S. Zeisberg, A. Olivereau, N. Oulha, L. Gheorghe, E. Slusanschi, et al., "A Trustworthy Architecture for Wireless Industrial Sensor Networks: Research Roadmap of EU TWISNet Trust and Security Project," in SysSec Workshop, 2011, pp. 63-66.

[73] P. Gaj, J. Jasperneite, and M. Felser, "Computer communication within industrial distributed environment, A survey," IEEE Transactions on Industrial Informatics, vol. 9, pp. 182-189, 2013.

[74] T. Sauter, "The three generations of field-level networksevolution and compatibility issues," IEEE Transactions on Industrial Electronics, vol. 57, pp. 3585-3595, 2010

[75] J. Garcia, F. R. Palomo, A. Luque, C. Aracil, J. M. Quero, D. Carrion, et al., "Reconfigurable distributed network control system for industrial plant automation," Industrial Electronics, IEEE Transactions on, vol. 51, pp. 1168-1180, 2004

[76] M. Bertoluzzo, G. Buja, and S. Vitturi, "Ethernet networks for factory automation," in Industrial Electronics,Proceedings of IEEE International Symposium on, 2002, pp. 175-180 vol.1.

[77] M. Felser, "Real time ethernet: Standardization and implementations," in Industrial Electronics (ISIE),IEEE International Symposium on, 2010, pp. 3766-3771

[78] J. C. Eidson and K. Lee, "Sharing a common sense of time," Instrumentation \& Measurement Magazine, IEEE, vol. 6, pp. 26-32, 2003.

[79] S.-L. Jamsa-Jounela, "Future trends in process automation," Annual Reviews in Control, vol. 31, pp. 211-220, 2007.

[80] D. Greenfield, Industrial Networks: Wired and Wireless: Automation World, Nov 2013.

[81] I. Akyildiz, W. Su, Y. Sankarasubramaniam, and E. Cayirci, "A Survey on Sensor Networks. IEEE Communications Magazine, 40, 102-114," ed, 2002.

[82] S. Petersen and S. Carlsen, "WirelessHART Versus ISA100.11a: The Format War Hits the Factory Floor,' Industrial Electronics Magazine, IEEE, vol. 5, pp. 23-34, 2011.

[83] P. Danielis, J. Skodzik, V. Altmann, E. B. Schweissguth, F. Golatowski, D. Timmermann, et al., "Survey on real-time communication via ethernet in industrial automation environments," in Emerging Technology and Factory Automation (ETFA), IEEE 2014, pp. 1-8.

[84] J.-D. Decotignie, "The many faces of industrial ethernet [past and present]," Industrial Electronics Magazine, IEEE, vol. 3, pp. 8-19, 2009

[85] G. Tapperson and T. A. Boyd, "Apparatus for providing non-redundant secondary access to field devices in a distributed control system," ed: Google Patents, 1998.

[86] P. S. Hoon, Y. R. Huan, J. Berge, and B. Sim, "Foundation Fieldbus high speed Ethernet (HSE) implementation," in Intelligent Control, 2002. Proceedings of IEEE International Symposium on, 2002, pp. 777-782.

[87] K. D. Krivoshein, "Process control configuration system for use with a profibus device network," ed: Google Patents, 2002.

[88] G. Prytz, "A performance analysis of EtherCAT and PROFINET IRT," in Emerging Technologies and Factory Automation, IEEE International Conference on, ETFA 2008, pp. 408-415.

[89] M. Felser, "Real-time ethernet-industry prospective," Proceedings of the IEEE, vol. 93, pp. 1118-1129, 2005.

[90] M. J. Miller and N. H. Vaidya, "A MAC protocol to reduce sensor network energy consumption using a wakeup radio," Mobile Computing, IEEE Transactions on, vol. 4, pp. 228-242, 2005.

[91] L. Gu and J. A. Stankovic, "Radio-Triggered Wake-Up Capability for Sensor Networks," in IEEE Real-Time and Embedded Technology and Applications Symposium, 2004, pp. 27-37.

[92] J. Shouling, L. Yingshu, and J. Xiaohua, "Capacity of dual-radio multichannel wireless sensor networks for continuous data collection," in INFOCOM, Proceedings IEEE, 2011, pp. 1062-1070.

[93] P. Zhibo, Y. Kan, J. Akerberg, and M. Gidlund, "An RTOS-based architecture for industrial wireless sensor network stacks with multi- processor support,” in Industrial Technology (ICIT), IEEE International Conference on, 2013, pp. 1216-1221.

[94] M. F. Khan, E. A. Felemban, S. Qaisar, and S. Ali, "Performance Analysis on Packet Delivery Ratio and End-to-End Delay of Different Network Topologies in Wireless Sensor Networks (WSNs), “ Mobile Adhoc and Sensor Networks (MSN), IEEE Ninth International Conference on, 2013, pp. 324-329.

[95] T. Instruments, What Is a Wireless Sensor Network (White Paper), 2012

[96] T O'donovan, J Brown, F Busching, A. Cardoso et al., "The GINSENG system for wireless monitoring and control: Design and deployment experiences," ACM Trans. Sen. Netw., vol. 10, pp. 1-40, 2013.

[97] P. Tung-Linh and K. Dong-Seong, "Lossy link-aware routing algorithm for ISA100.11a wireless networks," in Industrial Informatics (INDIN), 11th IEEE International Conference on, 2013, pp. 624-629.

[98] G. Wenqi, W. M. Healy, and Z. MengChu, "Impacts of 2.4-GHz ISM Band Interference on IEEE 802.15.4 Wireless Sensor Network Reliability in Buildings," Instrumentation and Measurement, IEEE Transactions on, vol. 61, pp. 2533-2544, 2012.

[99] S. S. Rizvi and C. Tae-Sun, "A survey of storage management in flash based data centric sensor devices in wireless sensor networks," in Communication Systems, Networks and Applications (ICCSNA), Second International Conference on, 2010, p. 1.

[100] M. Raza, G. Ahmed, N. M. Khan, M. Awais, and Q. Badar, "A comparative analysis of energy-aware routing protocols in wireless sensor networks," in Information and Communication Technologies (ICICT), International Conference on, 2011, pp. 1-5.

[101] L. Min, Y. Jianping, L. Jun, W. Yongan, and C. JieRen, "An efficient key management based on dynamic generation of polynomials for heterogeneous sensor networks," in Computer Engineering and Technology (ICCET), 2nd International Conference on, 2010, pp. V5-460-V5-464.

[102] F. Dobslaw, T. Zhang, and M. Gidlund, "End-to-End Reliability-aware Scheduling for Wireless Sensor Networks," Industrial Informatics, IEEE Transactions on, vol. PP, pp. 1-1, 2014

[103] F. Dobslaw, T. Zhang, and M. Gidlund, "Latency Improvement Strategies for Reliability-Aware Scheduling in Industrial Wireless Sensor Networks," International Journal of Distributed Sensor Networks, p. 10, 2015.

[104] W. Ikram, S. Petersen, P. Orten, and N. F. Thornhill, "Adaptive MultiChannel Transmission Power Control for Industrial Wireless Instrumentation,” IEEE Trans. Industrial Informatics, vol. 10, pp. 978-990, 2014.

[105] S. A. M. Atta ur Rehman Khan, Khizar Hayat, Samee Ullah Khan, "Clustering-based power-controlled routing for mobile wirelesssensor networks," NTERNATIONAL JOURNAL OF COMMUNICATION SYSTEMS, vol. 25, 2012.

[106] M. Raza, G. Ahmed Noor M Khan, "Power Control-based GRACE (PC-GRACE) Routing Protocol for Wireless Sensor Networks," Journal of Networking Technology, vol. 5, p. 137, 2014.

[107] M. Raza, G. Ahmed, and N. M. Khan, "Experimental evaluation of transmission power control strategies in wireless sensor networks," in Emerging Technologies (ICET), 2012 International Conference on, 2012, pp. 1-4

[108] Y. Kan, M. Gidlund, J. Akerberg, and M. Bjorkman, "Reliable real-time routing protocol for industrial wireless sensor and actuator networks," in Industrial Electronics and Applications (ICIEA), 2013 8th IEEE Conference on, 2013, pp. 1895-1901.

[109] K. Yu, Z. Pang, M. Gidlund, J. Akerberg,M. Bjorkman,"REALFLOW: Reliable Real-Time Flooding-Based Routing Protocol for Industrial Wireless Sensor Networks," International Journal of Distributed Sensor Networks, vol. 2014, p. 17, 2014.

[110] F. Barac, M. Gidlund, and Z. Tingting, "Ubiquitous, Yet Deceptive: Hardware-Based Channel Metrics on Interfered WSN Links," Vehicular Technology, IEEE Transactions on, vol. 64, pp. 1766-1778, 2015.

[111] G. Anastasi, M. Conti, and M. Di Francesco, "Extending the Lifetime of Wireless Sensor Networks Through Adaptive Sleep," Industrial Informatics, IEEE Transactions on, vol. 5, pp. 351-365, 2009.

[112] A. J. Banu and R. Velayutham, "Secure communication in Wireless Sensor Networks using AES algorithm with delay efficient sleep scheduling," in Emerging Trends in Computing, Communication and Nanotechnology (ICE-CCN), 2013 International Conference on, 2013, pp. 706-711.

[113] M. Barcelo Llado, A. Correa Vila, J. Lo Vetere, and A. Morell Perez, "Cooperative Multi-tree Sleep Scheduling for Surveillance in Wireless Sensor Networks," in Military Communications Conference, MILCOM 2013 IEEE, 2013, pp. 200-205.

[114] D. Diongue and O. Thiare, "ALARM: An energy aware sleep scheduling algorithm for lifetime maximization in wireless sensor networks," in 
Wireless Technology and Applications (ISWTA), 2013 IEEE Symposium on, 2013, pp. 74-79.

[115] Y. Jae Hyun and H. J. Kim, "Predictive Target Detection and Sleep Scheduling for Wireless Sensor Networks," in Systems, Man, and Cybernetics (SMC), 2013 IEEE International Conference on, 2013, pp. 362-367.

[116] G. Peng, J. Tao, Z. Qian, and Z. Kui, "Sleep Scheduling for Critical Event Monitoring in Wireless Sensor Networks," Parallel and Distributed Systems, IEEE Transactions on, vol. 23, pp. 345-352, 2012.

[117] L. Xueyan, C. Hongbin, Z. Feng, and L. Xiaohuan, "A range-based sleep scheduling algorithm for desired area coverage in solar-powered wireless sensor networks," in Wireless Communications and Signal Processing (WCSP), 2014 Sixth International Conference on, 2014, pp. 1-6.

[118] K. Jaffres-Runser, C. Comaniciu, J.-M. Gorce, and Z. Ruifeng, "UGRAB: A utility-based gradient broadcasting algorithm for wireless sensor networks," in Military Communications Conference, 2009. MILCOM 2009. IEEE, 2009, pp. 1-7.

[119] F. Ye, G. Zhong, S. Lu, and L. Zhang, "Gradient broadcast: A robust data delivery protocol for large scale sensor networks," Wireless Networks, vol. 11, pp. 285-298, 2005.

[120] L. Hao Wen, Z. Li, H. Xinchang, and T. Murata, "A Wireless Sensor Network Topology Design Method Based on Negotiable Evolutionary Algorithm," in Genetic and Evolutionary Computing (ICGEC), 2012 Sixth International Conference on, 2012, pp. 207-210.

[121] M. Sultana, S. R. Mahmud, L. Ansari, and N. A. Khan, "Delaunay Triangulation and st-numbering in Wireless Sensor Network topology," in Electrical Engineering and Information \& Communication Technology (ICEEICT), 2014 International Conference on, 2014, pp. 1-7.

[122] I. Bahaddur, C. L. Triveni, and P. C. Srikanth, "Novel Defense mechanism against data flooding attacks in ad hoc network," in Computing, Communications and Networking Technologies (ICCCNT),2013 Fourth International Conference on, 2013, pp. 1-6.

[123] A. Mohd, N. Aslam, W. Phillips, and W. Robertson, "A dual-mode energy efficient encryption protocol for wireless sensor networks," Ad Hoc Networks, vol. 11, pp. 2588-2604, 2013.

[124] A. Moh'd, N. Aslam, W. Phillips, W. Robertson, and H. Marzi, "SNSEC: a secure wireless sensor platform with hardware cryptographic primitives," Personal and ubiquitous computing, vol. 17, pp. 1051-1059, 2013.

[125] "ISO/IEC/IEEE International Standard - Information technology Telecommunications and information exchange between systems Local and metropolitan area networks - Specific requirements Part 11: Wireless LAN medium access control (MAC) and physical layer (PHY) specifications AMENDMENT 4," ISO/IEC/IEEE 880211:2012/Amd.4:2015(E) (Adoption of IEEE Std 802.11ac-2013), pp. 1430, 2015.

[126] Z. Shah, S. Rau, and A. Baig, "Throughput comparison of IEEE 802.11ac and IEEE 802.11n in an indoor environment with interference," in Telecommunication Networks and Applications Conference (ITNAC), 2015 International, 2015, pp. 196-201.

[127] "IEEE Standard for Information Technology-Telecommunications and information exchange between systems-Local and metropolitan area networks-Specific requirements Part 11: Wireless LAN Medium Access Control (MAC) and Physical Layer (PHY) specifications Amendment 10: Mesh Networking," IEEE Std 802.11s-2011 (Amendment to IEEE Std $802.11-2007$ as amended by IEEE $802.11 \mathrm{k}-2008$, IEEE $802.11 \mathrm{r}-$ 2008, IEEE 802.11y-2008, IEEE 802.11w-2009, IEEE 802.11n-2009, IEEE 802.11p-2010, IEEE 802.11z-2010, IEEE 802.11v-2011, and IEEE 802.11u-2011), pp. 1-372, 2011.

[128] "IEEE Draft Supplement to Standard [For] Information TechnologyTelecommunications and Information Exchange Between Systems-Local and Metropolitan Area Networks-Specific Requirements -Part 11: Wireless LAN Medium Access Control (MAC) and Physical Layer (PHY) Speci?Cations: Supplement to IEEE Std 802.11-1999," IEEE Std P802.11a/D7, p. 1, 1999

[129] "IEEE Standard for Information Technology- Telecommunications and Information Exchange Between Systems-Local and Metropolitan Area Networks-Specific Requirements-Part 11: Wireless LAN Medium Access Control (MAC) and Physical Layer (PHY) Specifications," IEEE Std 802.11-1997, pp. i-445, 1997.

[130] "IEEE Standard for Information technology- Local and metropolitan area networks- Specific requirements- Part 15.1a: Wireless Medium Access Control (MAC) and Physical Layer (PHY) specifications for Wireless Personal Area Networks (WPAN)," IEEE Std 802.15.1-2005 (Revision of IEEE Std 802.15.1-2002), pp. 1-700, 2005.

[131] J. S. Lee, Y. W. Su and C. C. Shen, "A Comparative Study of Wireless Protocols: Bluetooth, UWB, ZigBee, and Wi-Fi,” IECON 2007 - 33rd
Annual Conference of the IEEE Industrial Electronics Society, Taipei, 2007, pp. 46-51. doi: 10.1109/IECON.2007.4460126

[132] Abinayaa, V., and Anagha Jayan. "Case study on comparison of wireless technologies in industrial applications." International Journal of Scientific and Research Publications 4.2 (2014): 1-4.

[133] Ehrlich, Marco, Lukasz Wisniewski, and Jrgen Jasperneite. "State of the art and future applications of industrial wireless sensor networks." Proc. Kommunikation in der Automation (KommA) (2016): 80-87.

[134] C. Z. Yang, C. C. Chung and C. T. Lin, "On Scatternet Formation in Bluetooth Networks using Fuzzy Logic," 2007 IEEE Wireless Communications and Networking Conference, Kowloon, 2007, pp. 3141-3146. doi: 10.1109/WCNC.2007.580

[135] Rawat, Priyanka, et al. "Wireless sensor networks: a survey on recent developments and potential synergies." The Journal of supercomputing 68.1 (2014): 1-48.

[136] S. Promwong, B. Rankhamrat, T. Thavonsassanavong and S. Chaiyapong, "Ultra wireless transmission waveform measurement with IEEE802.15.3a," 2013 13th International Symposium on Communications and Information Technologies (ISCIT), Surat Thani, 2013, pp. 647-651. doi: 10.1109/ISCIT.2013.6645941

[137] S. Kato et al., "Single carrier transmission for multi-gigabit 60GHz WPAN systems," in IEEE Journal on Selected Areas in Communications, vol. 27 , no. 8, pp. 1466-1478, October 2009. doi: 10.1109/JSAC.2009.091016

[138] WirelessHART, H. C. Foundation, (2016). Available: http://en.hartcomm.org/main_article/wirelesshart.html

[139] F. Labeau, A. Agarwal, and B. Agba, "Comparative study of Wireless Sensor Network standards for application in Electrical Substations," in Computing, Communication and Security (ICCCS), 2015 International Conference on, 2015, pp. 1-5.

[140] G. Montenegro, N. Kushalnagar, J. Hui, and D. Culler, "Transmission of IPv6 packets over IEEE 802.15. 4 networks,” 2070-1721, 2007.

[141] Z. Shelby and C. Bormann, 6LoWPAN: The wireless embedded Internet vol. 43: John Wiley \& Sons, 2011.

[142] Zigbee Pro, Zigbee Alliance, (2012). Available: [http://www.zigbee.org/zigbee-for-developers/networkspecifications/zigbeepro/]

[143] X. Zhang and K. G. Shin, "Enabling coexistence of heterogeneous wireless systems: case for ZigBee and WiFi," in Proceedings of the Twelfth ACM International Symposium on Mobile Ad Hoc Networking and Computing, 2011, p. 6.

[144] L. Shizhuang, L. Jingyu, and F. Yanjun, "ZigBee Based Wireless Sensor Networks and Its Applications in Industrial," in Automation and Logistics, 2007 IEEE International Conference on, 2007, pp. 1979-1983.

[145] ISA100.11a Historical Background. Available: [https://www.isa.org/store/products/product-detail/?productId=118261], 6LoWPAN [http://orbigo.net/2011/06/6lowpan-third-part-history-of-thestandard/?ckattempt $=1]$

[146] WirelessHART, IEC Approval, (2008). Available: [http://hartcomm.org/ hcf/news/pr2010/WirelessHART_approved_by_IEC.html]

[147] IETF 6LoWPAN, Work Group Approval, (2015). Available: [http://orbigo.net/2011/06/6lowpan-third-part-history-of-the-standard/?ck attempt=1]

[148] IWSN Global Market Stats, (2015). Available: http://www.prnewswire com/news-releases/wireless-sensors-technologies-and-global-markets3000859 22.html

[149] V. C. Gungor and G. P. Hancke, Industrial wireless sensor networks: Applications, protocols, and standards: CRC Press, 2013.

[150] DustNetworks, "LTC5800-WHM SmartMesh wirelessHART Node Wireless Mote Datasheet," ed, 2013.

[151] DustNetworks, "LTP5900-WHM SmartMesh wirelessHART Node Wireless Mote Datasheet," ed, 2014

[152] "Thread Networking Protocol—NXP," 2016.

[153] NXP, "Kinetis KW2x Wireless MCUs Datasheet," ed, 2015.

[154] NXP, "JN517x, IEEE802.15.4 Wireless Microcontroller Datasheet," ed, November 2016.

[155] SiliconLabs, "EM35x System-on-Chip (SoC) / Network Co-Processor (NCP) for ZigBee Datasheet," ed, 2013

[156] OpenMote, OpenMote Devices, (December 2016) Available: http://www.openmote.com/

[157] TexasInstruments, "CC2538 Powerful Wireless Microcontroller System-On-Chip for 2.4-GHz IEEE 802.15.4, 6LoWPAN, and ZigBee," ed, April 2015.

[158] RE-Mote, Zolertia systems, (2016). Available: http://zolertia.io/product/hardware/re-mote 
[159] TexasInstruments, "CC1200 Low-Power, High-Performance RF Transceiver," ed, October 2014.

[160] Z1, Zolertia Systems, (2016). Available: http://zolertia.io/z1

[161] Honeywell, "XYR 6000 Wireless Input Transmitter Datasheet," ed, 2010.

[162] Microchip, "RN1810/RN1810E, 2.4 GHz IEEE 802.11b/g/n Wireless Module Datasheet," ed, 2016.

[163] R. Kling, R. Adler, J. Huang, V. Hummel, and L. Nachman, "Intel mote: Sensor network technology for industrial applications," Proceedings of SPOTS, IPSN05, 2005.

[164] Crossbow, "Imote2, High-performance Wireless Sensor Network Node Datasheet," ed, 2005

[165] R. P. Narayanan, T. V. Sarath, and V. V. Vineeth, "Survey on Motes Used in Wireless Sensor Networks: Performance \& Parametric Analysis," Wireless Sensor Network, vol. 8, p. 67, 2016.

[166] New Generation of Waspmote Sensor Nodes, Libelium, (2016) Available: http://www.libelium.com/libelium-launches-new-generationof-waspmote-sensor-nodes/

[167] P. D. Marketing, Willow Technologies, (2016). Available: http://www.willow.co.uk/html/telosb_mote_platform.php

[168] Wireless Sensor Networks, MEMSIC Inc, (2016). Available: http://www.memsic.com/wireless-sensor-networks/

[169] SunSPOT Sensor Motes, SunSPOTWorld, (2016). Available: http://sunspotdev.org/

[170] Digi Product Details, Digi International, (2016). Available: http://www.digi.com/support/productdetail?pid=4357

[171] JN5139-Z01-M/00R1T, NXP Semiconductors RF/IF and RFID, (2016). Available: http://www.digikey.com/product-detail/en/JN5139-Z01-M

[172] Texas Instruments, "CC2480 2.4 GHz Zigbee Radio datasheet," ed, 2015

[173] Texas Instruments, "CC2420, 2.4 GHz IEEE 802.15.4 / ZigBee-ready RF Transceiver Datasheet," ed, 2014

[174] Texas Instruments, "CC1000, Single Chip Very Low Power RF Transceiver Datasheet," ed, 2009

[175] nordic Semiconductor, "nRF905, Sub 1-GHz RF Ultra Low Power Single chip 433/868/915 MHz Transceiver Datasheet," ed, June 2004

[176] Emerson Technologies, (December 2015). Available: http://www.emer sonindustrial.com/en-US/industries/Pages/industries.aspx

[177] GE Industrial, (December 2015). Available: http://www.geindustrial.c om/products

[178] ABB, (December 2015). Available: http://www.abb.com/ProductGuide /Alphabetical.aspx

[179] ENDRESS, (December 2015). Available: http://www.uk.endress.com len

[180] Siemens, (December 2015). Available: http://www.siemens.co.uk/ent ry/en/

[181] Yokogawa, (December 2015). Available: http://www.yokogawa.com/

[182] Yamatake, (December 2015). Available: http://www.yamatake.co/

[183] Invensys, (December 2015). Available: http://iom.invensys.com/EN/ pages/home.aspx

[184] Mitsubishi Corp, (Dec 2015). Available: http://www.mitsubishicorp .com/jp/en/index.html

[185] Honeywell, (December 2015). Available: https://honeywell.com/Pages /Home.aspx

[186] P. Naik and K. M. Sivalingam, "A survey of MAC protocols for sensor networks," in Wireless sensor networks, ed: Springer, 2004, pp. 93-107.

[187] A. Roy and N. Sarma, "Energy saving in MAC layer of wireless sensor networks: a survey," in National Workshop in Design and Analysis of Algorithm (NWDAA), Tezpur University, India, 2010.

[188] K. Kredo and P. Mohapatra, "Medium access control in wireless sensor networks," Computer networks, vol. 51, pp. 961-994, 2007.

[189] K. Langendoen, "Medium access control in wireless sensor networks," Medium access control in wireless networks, vol. 2, pp. 535-560, 2008.

[190] G. Anastasi, M. Conti, and M. Di Francesco, "A comprehensive analysis of the MAC unreliability problem in IEEE 802.15.4 wireless sensor networks," Industrial Informatics, IEEE Transactions on, vol. 7, pp. 52-65, 2011.

[191] P. Pangun, P. Di Marco, C. Fischione, and K. H. Johansson, "Modeling and Optimization of the IEEE 802.15.4 Protocol for Reliable and Timely Communications," Parallel and Distributed Systems, IEEE Transactions on, vol. 24, pp. 550-564, 2013.

[192] H. Shibo, C. Jiming, C. Peng, G. Yu, H. Tian, and S. Youxian, "Maintaining Quality of Sensing with Actors in Wireless Sensor Networks," Parallel and Distributed Systems, IEEE Transactions on, vol. 23, pp. 16571667,2012
[193] A.W. Chen, B. Shih, C. Chang, and C. Chen, "Enhanced MAC channel selection to improve performance of IEEE 802.15. 4," International Journal of Innovative Computing, Information and Control, vol. 6, pp. 5511-5526, 2010

[194] M. D. Francesco, G. Anastasi, M. Conti, S. K. Das, and V. Neri, "Reliability and energy-efficiency in IEEE 802.15. 4/ZigBee sensor networks: an adaptive and cross-layer approach," Selected Areas in Communications, IEEE Journal on, vol. 29, pp. 1508-1524, 2011.

[195] S.e. Yoo, P. K. Chong, D. Kim, Y. Doh, M.-L. Pham, E. Choi, et al., "Guaranteeing real-time services for industrial wireless sensor networks with IEEE 802.15. 4," Industrial Electronics, IEEE Transactions on, vol. 57, pp. 3868-3876, 2010.

[196] N. Marchenko, T. Andre, G. Brandner, W. Masood, and C. Bettstetter, "An experimental study of selective cooperative relaying in industrial wireless sensor networks," Industrial Informatics, IEEE Transactions on, vol. 10, pp. 1806-1816, 2014.

[197] S. C. Ergen and P. Varaiya, "TDMA scheduling algorithms for wireless sensor networks," Wireless Networks, vol. 16, pp. 985-997, 2010.

[198] W. Shen, T. Zhang, M. Gidlund, and F. Dobslaw, "SAS-TDMA: A source aware scheduling algorithm for real-time communication in industrial wireless sensor networks," Wireless networks, vol. 19, pp. 1155 1170, 2013.

[199] M. Yan, K.-Y. Lam, S. Han, E. Chan, Q. Chen, P. Fan, et al., "Hypergraph-based data link layer scheduling for reliable packet delivery in wireless sensing and control networks with end-to-end delay constraints," Information Sciences, vol. 278, pp. 34-55, 2014.

[200] C. L. Chang and K. Y. Ho, "Slot assignment for TDMA MAC in industrial wireless sensor network," in 2016 IEEE/ACIS 15th International Conference on Computer and Information Science (ICIS), 2016, pp. 1-5.

[201] L. F. Van Hoesel and P. J. Havinga, "A lightweight medium access protocol (LMAC) for wireless sensor networks: Reducing preamble transmissions and transceiver state switches," 2004.

[202] M. Handy, M. Haase, and D. Timmermann, "Low energy adaptive clustering hierarchy with deterministic cluster-head selection," in Mobile and Wireless Communications Network, 2002. 4th International Workshop on, 2002, pp. 368-372.

[203] V. Namboodiri and A. Keshavarzian, "Alert: An adaptive low-latency event-driven mac protocol for wireless sensor networks," in Proceedings of the 7th international conference on Information processing in sensor networks, 2008, pp. 159-170.

[204] J. Zhao, Y. Qin, D. Yang, and Y. Rao, "A source aware scheduling algorithm for time-optimal convergecast," International Journal of Distributed Sensor Networks, 2014.

[205] F. Dobslaw, T. Zhang, and M. Gidlund, "Latency Improvement Strategies for Reliability-Aware Scheduling in Industrial Wireless Sensor Networks," International Journal of Distributed Sensor Networks, 2015.

[206] C. Jiming, Y. Qing, C. Bo, S. Youxian, F. Yanfei, and S. Xuemin, "Dynamic Channel Assignment for Wireless Sensor Networks: A Regret Matching Based Approach," Parallel and Distributed Systems, IEEE Transactions on, vol. 26, pp. 95-106, 2015.

[207] L. F. de Moraes and R. S. Silva, "Analysis of multichannel wireless networks With priority-based polling MAC protocols," in Wireless Days (WD), 2014 IFIP, 2014, pp. 1-6.

[208] B. Andersson, N. Pereira, W. Elmenreich, E. Tovar, F. Pacheco and N. Cruz, "A Scalable and Efficient Approach for Obtaining Measurements in CAN-Based Control Systems," in IEEE Transactions on Industrial Informatics, vol. 4, no. 2, pp. 80-91, May 2008.

[209] C. Schurgers, V. Tsiatsis, S. Ganeriwal, and M. Srivastava, "Optimizing sensor networks in the energy-latency-density design space," IEEE transactions on mobile computing, vol. 1, pp. 70-80, 2002.

[210] L. Tang, Y. Sun, O. Gurewitz, and D. B. Johnson, "PW-MAC: An energy-efficient predictive-wakeup MAC protocol for wireless sensor networks," in 2011 Proceedings IEEE INFOCOM, 2011, pp. 1305-1313.

[211] M. C. Vuran and I. F. Akyildiz, "Spatial correlation-based collaborative medium access control in wireless sensor networks," IEEE/ACM Transactions On Networking, vol. 14, pp. 316-329, 2006.

[212] J. Polastre, J. Hill, and D. Culler, "Versatile low power media access for wireless sensor networks," in Proceedings of the 2nd international conference on Embedded networked sensor systems, 2004, pp. 95-107.

[213] X. Shi and G. Stromberg, "SyncWUF: An ultra low-power MAC protocol for wireless sensor networks," IEEE Transactions on Mobile Computing, vol. 6, pp. 115-125, 2007.

[214] J. Ansari, X. Zhang, and P. Mhnen, "Poster abstract: Traffic aware medium access control protocol for wireless sensor networks," in Proceedings of the third European Conference on Computer Systems (EuroSys), 2008 
[215] E.Y. Lin, J. M. Rabaey, and A. Wolisz, "Power-efficient rendez-vous schemes for dense wireless sensor networks," in Communications, 2004 IEEE International Conference on, 2004, pp. 3769-3776.

[216] W. Ye, J. Heidemann, and D. Estrin, "An energy-efficient MAC protocol for wireless sensor networks," in INFOCOM 2002. Twenty-First Annual Joint Conference of the IEEE Computer and Communications Societies. Proceedings. IEEE, 2002, pp. 1567-1576.

[217] P. Lin, C. Qiao, and X. Wang, "Medium access control with a dynamic duty cycle for sensor networks," in Wireless Communications and Networking Conference, 2004 IEEE WCNC, 2004, pp. 1534-1539.

[218] L. Bao and J. Garcia-Luna-Aceves, "A new approach to channel access scheduling for ad hoc networks," in Proceedings of the 7th annual international conference on Mobile computing and networking, 2001, pp. 210-221.

[219] B. Yahya and J. Ben-Othman, "An energy efficient hybrid medium access control scheme for wireless sensor networks with quality of service guarantees," in IEEE GLOBECOM, 2008 IEEE Global Telecommunications Conference, 2008, pp. 1-5.

[220] G. Zhou, C. Huang, T. Yan, T. He, J. A. Stankovic, and T. F. Abdelzaher, "MMSN: Multi-Frequency Media Access Control for Wireless Sensor Networks," in Infocom, 2006, pp. 1-13.

[221] Y. Wu, J. A. Stankovic, T. He, and S. Lin, "Realistic and efficient multichannel communications in wireless sensor networks," in INFOCOM 2008. The 27th Conference on Computer Communications. IEEE, 2008.

[222] Y. Kim, H. Shin, and H. Cha, "Y-MAC: An energy-efficient multichannel MAC protocol for dense wireless sensor networks," in Proceedings of the 7 th international conference on Information processing in sensor networks, 2008, pp. 53-63.

[223] M. Salajegheh, H. Soroush, and A. Kalis, "HYMAC: Hybrid TDMA/FDMA medium access control protocol for wireless sensor networks," in 2007 IEEE 18th International Symposium on Personal, Indoor and Mobile Radio Communications, 2007, pp. 1-5.

[224] J. Lin, W. Liang, H. Yu, and Y. Xiao, "Polling in the frequency domain: a new MAC protocol for industrial wireless network for factory automation," International Journal of Ad Hoc and Ubiquitous Computing, vol. 20, pp. 211-222, 2015.

[225] K. Jamieson, H. Balakrishnan, and Y. Tay, "Sift: A MAC protocol for event-driven wireless sensor networks," in European Workshop on Wireless Sensor Networks, 2006, pp. 260-275.

[226] A. Boukerche, Handbook of algorithms for wireless networking and mobile computing: CRC Press, 2005.

[227] G. Lu, B. Krishnamachari, and C. S. Raghavendra, "An adaptive energy-efficient and low-latency MAC for data gathering in wireless sensor networks," in Parallel and Distributed Processing Symposium, 2004 Proceedings. 18th International, 2004, p. 224.

[228] T. Van Dam and K. Langendoen, "An adaptive energy-efficient MAC protocol for wireless sensor networks," in Proceedings of the 1st international conference on Embedded networked sensor systems, 2003, pp. 171-180.

[229] Y.C. Chang, J.-R. Jiang, J.-P. Sheu, and H.-Y. Shih, "ADCA: an asynchronous duty cycle adjustment MAC protocol for wireless sensor networks," in IEEE GLOBECOM, 2008 IEEE Global Telecommunications Conference, 2008, pp. 1-5.

[230] Y. Liu, I. Elhanany, and H. Qi, “An energy-efficient QoS-aware media access control protocol for wireless sensor networks," in IEEE International Conference on Mobile Adhoc and Sensor Systems Conference, 2005, pp. 3 pp.-191.

[231] A. Farhad, Y. Zia, S. Farid, and F. B. Hussain, "A traffic aware dynamic super-frame adaptation algorithm for the IEEE 802.15.4 based networks," in 2015 IEEE Asia Pacific Conference on Wireless and Mobile (APWiMob), 2015, pp. 261-266.

[232] H. Pham and S. Jha, "An adaptive mobility-aware MAC protocol for sensor networks (MS-MAC)," in Mobile Ad-hoc and Sensor Systems, 2004 IEEE International Conference on, 2004, pp. 558-560.

[233] R. Yadav, S. Varma, and N. Malaviya, "Optimized medium access control for wireless sensor network," IJCSNS International Journal of Computer Science and Network Security, vol. 8, pp. 334-338, 2008.

[234] T. Zheng, S. Radhakrishnan, and V. Sarangan, "PMAC: an adaptive energy-efficient MAC protocol for wireless sensor networks," in 19th IEEE International Parallel and Distributed Processing Symposium, 2005, p. 8 pp.

[235] L. Guntupalli and F. Y. Li, "DTMC modeling for performance evaluation of DW-MAC in wireless sensor networks," in 2016 IEEE Wireless Communications and Networking Conference, 2016, pp. 1-6.

[236] A. El-Hoiydi, "Spatial TDMA and CSMA with preamble sampling for low power ad hoc wireless sensor networks," in Computers and
Communications, 2002. Proceedings. ISCC 2002. Seventh International Symposium on, 2002, pp. 685-692.

[237] V. Rajendrian, K. Obraczka, and J. Garcia-Luna-Aceves, "Energyefficient, collision free medium access control for woreless sensor network," in Proceedings of ACM SenSys 2003, 2003, pp. 181-192.

[238] R. Ijnong, M. A. Ajir Warrier, and M. Jeongki, "ZMAC: a hybrid MAC for Wireless Sensor Networks," in Prec. of 3rd ACM Conference on Embedded Networked Sensor Systems (SenSys 2005) Los Angeles. CA. USA, 2005

[239] M. Adamou, I. Lee, and I. Shin, "An energy efficient real-time medium access control protocol for wireless ad-hoc networks," REPORTUNIVERSITY OF YORK DEPARTMENT OF COMPUTER SCIENCE YCS, pp. 5-8, 2001.

[240] H. Wang, X. Zhang, and A. Khokhar, "WSN05-6: An Energy-Efficient Low-Latency MAC Protocol for Wireless Sensor Networks," in IEEE Globecom 2006, pp. 1-5.

[241] S. Biaz and Y. D. Barowski, "GANGS: an energy efficient MAC protocol for sensor networks," in Proceedings of the 42nd annual Southeast regional conference, 2004, pp. 82-87.

[242] Z. Liu, D. Dragomirescu, G. D. Costa, and T. Monteil, "Dynamic multichannel allocation mechanism for wireless multimedia sensor networks," in 2016 Wireless Days (WD), 2016, pp. 1-6.

[243] N. Nasser, L. Karim, and T. Taleb, "Dynamic Multilevel Priority Packet Scheduling Scheme for Wireless Sensor Network," IEEE Transactions on Wireless Communications, vol. 12, pp. 1448-1459, 2013.

[244] K.H. Phung, B. Lemmens, M. Goossens, A. Nowe, L. Tran, and K. Steenhaut, "Schedule-based multi-channel communication in wireless sensor networks: A complete design and performance evaluation," $\mathrm{Ad}$ Hoc Networks, vol. 26, pp. 88-102, 2015.

[245] J. Wun-Cheol and L. Junhee, "Performance evaluation of IEEE 802.15.4e DSME MAC protocol for wireless sensor networks," in The First IEEE Workshop on Enabling Technologies for Smartphone and Internet of Things (ETSIoT), 2012, pp. 7-12.

[246] S. Meninger, J. O. Mur-Miranda, R. Amirtharajah, A. P. Chandrakasan, and J. H. Lang, "Vibration-to-electric energy conversion," Very Large Scale Integration (VLSI) Systems, IEEE Transactions on, vol. 9, pp. 6476, 2001.

[247] S. Chalasani and J. M. Conrad, "A survey of energy harvesting sources for embedded systems," in Southeastcon, IEEE, 2008, pp. 442-447.

[248] V. Raghunathan, A. Kansal, J. Hsu, J. Friedman, and M. Srivastava, "Design considerations for solar energy harvesting wireless embedded systems," in Proceedings of the 4th international symposium on Information processing in sensor networks, 2005, p. 64

[249] J. P. Amaro, F. J. T. E. Ferreira, R. Cortesao, and J. Landeck, "Energy harvesting for Zigbee compliant Wireless Sensor Network nodes," in IECON 2012, 38th Annual Conference on IEEE Industrial Electronics Society, 2012, pp. 2583-2588.

[250] R. Vullers, R. Schaijk, H. Visser, J. Penders, and C. Hoof, "Energy harvesting for autonomous wireless sensor networks," IEEE Solid-State Circuits Magazine, vol. 2, pp. 29-38, 2010.

[251] A. Paidimarri, N. Ickes, and A. Chandrakasan, "A+ 10dBm $2.4 \mathrm{GHz}$ Transmitter with sub-400pW Leakage and $43.7 \%$ System Efficiency," 2015.

[252] M.L. Ku, Y. Chen, and K. R. Liu, "Data-driven stochastic models and policies for energy harvesting sensor communications," IEEE Journal on Selected Areas in Communications, vol. 33, pp. 1505-1520, 2015.

[253] W. S. Wang, T. O'Donnell, N. Wang, M. Hayes, B. O'Flynn, and C. O'Mathuna, "Design considerations of sub-mW indoor light energy harvesting for wireless sensor systems," ACM Journal on Emerging Technologies in Computing Systems (JETC), vol. 6, p. 6, 2010.

[254] Y. K. Tan and S. K. Panda, "Energy harvesting from hybrid indoor ambient light and thermal energy sources for enhanced performance of wireless sensor nodes," IEEE Transactions on Industrial Electronics, vol. 58, pp. 4424-4435, 2011.

[255] H. J. Visser and R. J. Vullers, "RF energy harvesting and transport for wireless sensor network applications: Principles and requirements,' Proceedings of the IEEE, vol. 101, pp. 1410-1423, 2013.

[256] S. Kim, R. Vyas, J. Bito, K. Niotaki, A. Collado, A. Georgiadis, et al., "Ambient RF energy-harvesting technologies for self-sustainable standalone wireless sensor platforms," Proceedings of the IEEE, vol. 102, pp. 1649-1666, 2014.

[257] I. Krikidis, S. Timotheou, S. Nikolaou, G. Zheng, D. W. K. Ng, and R. Schober, "Simultaneous wireless information and power transfer in modern communication systems," IEEE Communications Magazine, vol. 52, pp. 104-110, 2014.

[258] S. Boisseau, G. Despesse, and B. A. Seddik, "Electrostatic conversion for vibration energy harvesting," arXiv preprint arXiv:1210.5191, 2012. 
[259] W. K. Seah, Z. A. Eu, and H.-P. Tan, "Wireless sensor networks powered by ambient energy harvesting (WSN-HEAP)-Survey and challenges," in Wireless Communication, Vehicular Technology, Information Theory and Aerospace \& Electronic Systems Technology, Wireless VITAE 2009, 1st International Conference on, 2009, pp. 1-5.

[260] H. Shibo, C. Jiming, J. Fachang, D. K. Y. Yau, X. Guoliang, and S. Youxian, "Energy Provisioning in Wireless Rechargeable Sensor Networks," Mobile Computing, IEEE Transactions on, vol. 12, pp. 19311942, 2013.

[261] A. Berger, L. B. Hormann, C. Leitner, S. B. Oswald, P. Priller, and A. Springer, "Sustainable energy harvesting for robust wireless sensor networks in industrial applications," in Sensors Applications Symposium (SAS), 2015 IEEE, 2015, pp. 1-6.

[262] J. Azevedo and F. Santos, "Energy harvesting from wind and water for autonomous wireless sensor nodes," IET Circuits, Devices \& Systems, vol. 6, pp. 413-420, 2012.

[263] P. Cuffe, P. Smith, and A. Keane, "Transmission system impact of wind energy harvesting networks," IEEE Transactions on Sustainable Energy, vol. 3, pp. 643-651, 2012.

[264] C. Park and P. H. Chou, "Ambimax: Autonomous energy harvesting platform for multi-supply wireless sensor nodes," in 2006 3rd annual IEEE communications society on sensor and ad hoc communications and networks, 2006, pp. 168-177.

[265] J. Jessen, M. Venzke, and V. Turau, "Design considerations for a universal smart energy module for energy harvesting in wireless sensor networks," in Proceedings of the 9th Workshop for Intelligent Solutions in Embedded Systems (WISES), 2011, pp. 35-40.

[266] B. Ulmen, P. Desai, S. Moghaddam, G. Miley, and R. Masel, "Development of diode junction nuclear battery using $63 \mathrm{Ni}$," Journal of radioanalytical and nuclear chemistry, vol. 282, pp. 601-604, 2009.

[267] B. J. Hansen, Y. Liu, R. Yang, and Z. L. Wang, "Hybrid nanogenerator for concurrently harvesting biomechanical and biochemical energy," ACS nano, vol. 4, pp. 3647-3652, 2010

[268] H. Long, Y. Liu, Y. Wang, R. P. Dick, and H. Yang, "Battery allocation for wireless sensor network lifetime maximization under cost constraints," in Proceedings of the International Conference on ComputerAided Design, 2009, pp. 705-712

[269] K. Mikhaylov and J. Tervonen, "Node's Power Source Type Identification in Wireless Sensor Networks," in Broadband and Wireless Computing, Communication and Applications (BWCCA), 2011 International Conference on, 2011, pp. 521-525.

[270] Sun SPOT Mainboard Technical Datasheet Revison 8.0, (January 2016). Available: http://sunspotdev.org/docs/Yellow/eSPOT8ds.pdf

[271] A Practical Guide to Battery Technologies for Wireless Sensor Networking Sensors, (2016). Available: http://www.sensorsmag.com/networking-communications/batteries/apractical-guide-battery-technologies-wireless-sensor-netwo-1499

[272] Energizer, "Alkaline AA (E91 Battery),".

[273] Energizer, "Cylindrical Primary Lithium Chemical System: Lithium/Iron Disulfide (Li/FeS2) (L91),",

[274] Energizer, "Lithium 9V, Lithium-Manganese Dioxide (Li/MnO2) (LA522)

[275] S. H. Lee and L. Choi, "SPEED-MAC: speedy and energy efficient data delivery MAC protocol for real-time sensor network applications," Wireless Networks, vol. 21, pp. 883-898, 2015.

[276] Y. Sun, S. Du, O. Gurewitz, and D. B. Johnson, "DW-MAC: a low latency, energy efficient demand-wakeup MAC protocol for wireless sensor networks," in Proceedings of the 9th ACM international symposium on Mobile ad hoc networking and computing, 2008, pp. 53-62.

[277] N. Aslam, W. Philips, W. Robertson, and S. Sivakumar, "A multicriterion optimization technique for energy efficient cluster formation in wireless sensor networks," Information Fusion, vol. 12, pp. 202-212, 2011.

[278] L. Grobe, A. Paraskevopoulos, J. Hilt, D. Schulz, F. Lassak, F Hartlieb, et al., "High-speed visible light communication systems," IEEE Communications Magazine, vol. 51, pp. 60-66, 2013.

[279] D. C. O’Brien, L. Zeng, H. Le-Minh, G. Faulkner, J. W. Walewski, and S. Randel, "Visible light communications: Challenges and possibilities," in Personal, Indoor and Mobile Radio Communications, 2008. PIMRC 2008. IEEE 19th International Symposium on, 2008, pp. 1-5.

[280] T. M. Chiwewe, C. F. Mbuya, and G. P. Hancke, "Using Cognitive Radio for Interference-Resistant Industrial Wireless Sensor Networks: An Overview," Industrial Informatics, IEEE Transactions on, vol. 11, pp. 1466-1481, 2015

[281] G. P. Joshi, S. Y. Nam, and S. W. Kim, "Cognitive radio wireless sensor networks: applications, challenges and research trends," Sensors, vol. 13, pp. 11196-11228, 2013.
[282] S. Li, L. D. Xu, and X. Wang, "Compressed Sensing Signal and Data Acquisition in Wireless Sensor Networks and Internet of Things,' IEEE Transactions on Industrial Informatics, vol. 9, pp. 2177-2186, 2013.

[283] P. T. A. Quang and D.-S. Kim, "Enhancing real-time delivery of gradient routing for industrial wireless sensor networks," Industrial Informatics, IEEE Transactions on, vol. 8, pp. 61-68, 2012.

[284] D. Goyal and M. R. Tripathy, "Routing protocols in wireless sensor networks: a survey," in Advanced Computing \& Communication Technologies (ACCT), 2012 Second International Conference on, 2012, pp. 474-480.

[285] Gubbi, Jayavardhana, et al. "Internet of Things (IoT): A vision, architectural elements, and future directions." Future generation computer systems 29.7 (2013): 1645-1660.

[286] $\mathrm{Da} \mathrm{Xu}, \mathrm{Li}, \mathrm{Wu} \mathrm{He}$, and Shancang Li. "Internet of things in industries: A survey." IEEE Transactions on industrial informatics 10.4 (2014): 22332243.

[287] D. Guinard, V. Trifa, S. Karnouskos, P. Spiess, and D. Savio, "Interacting with the soa-based internet of things: Discovery, query, selection, and ondemand provisioning of web services, IEEE Trans. Serv. Comput. vol. 3, no. 3, pp. 223235, Jul./Sep. 2010.

[288] C. Flgel and V. Gehrmann, "Scientific workshop 4: Intelligent objects for the internet of things: Internet of things-application of sensor networks in logistics, Commun. Comput. Inf. Sci., vol. 32, pp. 1626, 2009.

[289] L. Atzori, A. Iera, and G. Morabito, "The internet of things: A survey, Comput. Netw., vol. 54, no. 15, pp. 27872805, 2010

[290] D. Miorandi, S. Sicari, F. De Pellegrini, and I. Chlamtac, "Internet of things: Vision, applications and research challenges, Ad Hoc Netw., vol. 10, no. 7 , pp. $14971516,2012$.

[291] Watteyne, Thomas, et al. "OpenWSN: a standardsbased lowpower wireless development environment." Transactions on Emerging Telecommunications Technologies 23.5 (2012): 480-493.

[292] Chang, Tengfei, et al. "OpenWSN \& OpenMote: Demo'ing a Complete Ecosystem for the Industrial Internet of Things." Sensing, Communication, and Networking (SECON), 2016 13th Annual IEEE International Conference on. IEEE, 2016

[293] N. Kushalnagar, G. Montenegro, and C. P. P. Schumacher, IPv6 over Low-Power Wireless Personal Area Networks (6LoWPANs): Overview, Assumptions, Problem Statement, and Goals, IETF Std. RFC4919, August 2007.

[294] T. Winter, P. Thubert, A. Brandt, J. Hui, R. Kelsey, P. Levis, K. Pister, R. Struik, J. Vasseur, and R. Alexander, "RPL: IPv6 Routing Protocol for Low-Power and Lossy Networks", IETF Std. RFC6550, March 2012.

[295] Z. Shelby, K. Hartke, and C. Bormann, The Constrained Application Protocol (CoAP), IETF Std. RFC7252, June 2014.

[296] IETF Charter of IPv6 over Networks of Resourceconstrained Nodes (6lo) Working Group, (2017) Available: [https//datatracker.ietf.org/wg/6lo/charter]

[297] IETF: IPv6 over Networks of Resource-constrained Nodes (6lo), Active Internet Drafts (June 2017), Available: [https//datatracker.ietf.org/wg/6lo/documents]

[319] Melodia, Tommaso, et al. "Communication and coordination in wireless sensor and actor networks." IEEE transactions on mobile computing 6.10 (2007)

[298] J. Vu, C. Kottke, S. Nerreter, K. Habel, B. A, ttner, et al., "230 $\mathrm{Mbit} / \mathrm{s}$ via a wireless visible-light link based on OOK modulation of phosphorescent white LEDs," in Optical Fiber Communication (OFC), collocated National Fiber Optic Engineers Conference, 2010 Conference on (OFC/NFOEC), 2010, pp. 1-3.

[299] L. Zeng, D. C. O. Brien, H. L. Minh, G. E. Faulkner, K. Lee, D. Jung, et al., "High data rate multiple input multiple output (MIMO) optical wireless communications using white led lighting," IEEE Journal on Selected Areas in Communications, vol. 27, pp. 1654-1662, 2009.

[300] M. Hoa Le, D. O. Brien, G. Faulkner, L. Zeng, L. Kyungwoo, J. Daekwang, et al., " $80 \mathrm{Mbit} / \mathrm{s}$ Visible Light Communications using preequalized white LED," in Optical Communication, 2008. ECOC 2008. 34th European Conference on, 2008, pp. 1-2.

[301] J. Zhu, A. Waltho, X. Yang, and X. Guo, "Multi-radio coexistence: Challenges and opportunities," in Computer Communications and Networks, 2007. ICCCN 2007. Proceedings of 16th International Conference on, 2007 , pp. 358-364.

[302] S. Pollin, I. Tan, B. Hodge, C. Chun, and A. Bahai, "Harmful coexistence between 802.15. 4 and 802.11: A measurement-based study," in Cognitive Radio Oriented Wireless Networks and Communications, 3rd International Conference on, CrownCom, 2008, pp. 1-6.

[303] R. Gummadi, D. Wetherall, B. Greenstein, and S. Seshan, "Understanding and mitigating the impact of RF interference on 802.11 networks," 
ACM SIGCOMM Computer Communication Review, vol. 37, pp. 385396, 2007.

[304] A. Ahmad, S. Ahmad, M. H. Rehmani, and N. U. Hassan, "A survey on radio resource allocation in cognitive radio sensor networks," IEEE Communications Surveys \& Tutorials, vol. 17, pp. 888-917, 2015.

[305] G. A. Shah, V. C. Gungor, and O. B. Akan, "A cross-layer design for QoS support in cognitive radio sensor networks for smart grid applications," in 2012 IEEE International Conference on Communications (ICC), 2012, pp. 1378-1382.

[306] J. Silvestre-Blanes, L. Almeida, R. Marau, and P. Pedreiras, "Online QoS management for multimedia real-time transmission in industrial networks," Industrial Electronics, IEEE Transactions on, vol. 58, pp 1061-1071, 2011.

[307] J. Chen, X. Cao, P. Cheng, Y. Xiao, and Y. Sun, "Distributed collaborative control for industrial automation with wireless sensor and actuator networks," Industrial Electronics, IEEE Transactions on, vol. 57, pp. 4219-4230, 2010

[308] S. H. R. Bukhari, M. H. Rehmani, and S. Siraj, "A survey of channel bonding for wireless networks and guidelines of channel bonding for futuristic cognitive radio sensor networks," IEEE Communications Surveys \& Tutorials, vol. 18, pp. 924-948, 2016.

[309] O. B. Akan, O. B. Karli, and O. Ergul, "Cognitive radio sensor networks," IEEE network, vol. 23, pp. 34-40, 2009.

[310] T. Zheng, Y. Qin, H. Zhang, and S.-Y. Kuo, "A self-configurable power control algorithm for cognitive radio-based industrial wireless sensor networks with interference constraints," in 2012 IEEE International Conference on Communications (ICC), 2012, pp. 98-103.

[311] A. Asokan and R. AyyappaDas, "Survey on cognitive radio and cognitive radio sensor networks," in Electronics and Communication Systems (ICECS), 2014 International Conference on, 2014, pp. 1-7.

[312] I. F. Akyildiz, W.-Y. Lee, M. C. Vuran, and S. Mohanty, "NeXt generation/dynamic spectrum access/cognitive radio wireless networks: a survey," Computer networks, vol. 50, pp. 2127-2159, 2006.

[313] A. Fragkiadakis, V. Angelakis, and E. Z. Tragos, "Securing cognitive wireless sensor networks: a survey," International Journal of Distributed Sensor Networks, 2014.

[314] G. Bleser, D. Damen, A. Behera, G. Hendeby, K. Mura, M. Miezal, et al., "Cognitive learning, monitoring and assistance of industrial workflows using egocentric sensor networks," PloS one, vol. 10, p. e0127769, 2015.

[315] M. Eskola and T. Heikkil, "Classification of Radio Channel Disturbances for Industrial Wireless Sensor Networks," Ad Hoc Networks, 2016.

[316] A. Ahmad, S. Ahmad, M. H. Rehmani, and N. U. Hassan, "A survey on radio resource allocation in cognitive radio sensor networks," Communications Surveys \& Tutorials, IEEE, vol. 17, pp. 888-917, 2015.

[317] S. Zhuo, H. Shokri-Ghadikolaei, C. Fischione, and Z. Wang, "Adaptive congestion control in cognitive industrial wireless sensor networks," in Industrial Informatics (INDIN), 2015 IEEE 13th International Conference on, 2015, pp. 900-907.

[318] M. H. Rehmani, Cognitive Radio Sensor Networks: Applications, Architectures, and Challenges: Applications, Architectures, and Challenges: IGI Global, 2014.

[319] Melodia, Tommaso, et al. "Communication and coordination in wireless sensor and actor networks." IEEE transactions on mobile computing 6.10 (2007).

[320] Kurata, Narito, Shunsuke Saruwatari, and Hiroyuki Morikawa. "Ubiquitous structural monitoring using wireless sensor networks." Intelligent Signal Processing and Communications, 2006. ISPACS'06. International Symposium on. IEEE, 2006.

[321] Kim, Sukun, et al. "Health monitoring of civil infrastructures using wireless sensor networks." Proceedings of the 6th international conference on Information processing in sensor networks. ACM, 2007.

[322] Hou, Liqun, and Neil W. Bergmann. "Novel industrial wireless sensor networks for machine condition monitoring and fault diagnosis." IEEE Transactions on Instrumentation and Measurement 61.10 (2012): 27872798.

[323] Singh, Shio Kumar, M. P. Singh, and D. K. Singh. "A survey of energyefficient hierarchical cluster-based routing in wireless sensor networks." International Journal of Advanced Networking and Application (IJANA) 2.02 (2010): 570-580.

[324] Mukherjee, M., et al. "Sleep scheduling in industrial wireless sensor networks for toxic gas monitoring." IEEE Wireless Communications 99 (2017): 2-8.

[325] Zhu, Chunsheng, et al. "Collaborative location-based sleep scheduling for wireless sensor networks integratedwith mobile cloud computing." IEEE Transactions on Computers 64.7 (2015): 1844-1856.
[326] Kundu, Souvik, et al. "A modified differential evolution-based combined routing and sleep scheduling scheme for lifetime maximization of wireless sensor networks." Soft Computing-A Fusion of Foundations, Methodologies and Applications 19.3 (2015): 637-659.

[327] Mukherjee, Mithun, et al. "Low Control Overhead-Based Sleep Scheduling in Software-Defined Wireless Sensor Networks." High Performance Computing and Communications; IEEE 14th International Conference on Smart City; IEEE 2nd International Conference on Data Science and Systems (HPCC/SmartCity/DSS), 2016 IEEE 18th International Conference on. IEEE, 2016

[328] M. Raza, H. Le-Minh, N. Aslam, S. Hussain, "Deterministic Scheduling for Heterogeneous Sensing Deadlines in Industrial Wireless Sensor Networks", 2nd International Workshop on Sustainability and Green Technologies, 2017.

[329] Boaventura, Alrio Soares, and Nuno Borges Carvalho. "A low-power wakeup radio for application in WSN-based indoor location systems." International journal of wireless information networks (2013): 1-7.

[330] Wu, Mou, Liansheng Tan, and Naixue Xiong. "Data prediction, compression, and recovery in clustered wireless sensor networks for environmental monitoring applications." Information Sciences 329 (2016): 800818

[331] Yiu, Joseph, and Ian Johnson. Multi-core microcontroller design with Cortex-M processors and CoreSight SoC. Tech. rep. ARM, 2013

[332] Jie, Liu, Hemant Ghayvat, and S. C. Mukhopadhyay. "Introducing Intel Galileo as a development platform of smart sensor: Evolution, opportunities and challenges." Industrial Electronics and Applications (ICIEA), 2015 IEEE 10th Conference on. IEEE, 2015.

[333] Grail Semiconductor, Inc. v. Mitsubishi Electric \& Electronics USA, Inc., 225 Cal. App. 4th 786, 170 Cal. Rptr. 3d 581 (Ct. App. 2014).

[334] Babbar, Khushbu, Kusum Lata Jain, and G. N. Purohit. "Implementation of energy efficient coverage aware routing protocol for wireless sensor network using genetic algorithm." Int. J. Found. Comput. Sci. Technol 5 (2015): 23-34.

[335] Jing, Zhang, et al. "TQS-DP: A lightweight and active mechanism for fast scheduling based on WSN operating system TinyOS." Control and Decision Conference (CCDC), 2015 27th Chinese. IEEE, 2015.

[336] Botman, Franois, et al. "Bellevue: a 50MHz variable-width SIMD 32bit microcontroller at $0.37 \mathrm{~V}$ for processing-intensive wireless sensor nodes." Circuits and Systems (ISCAS), 2014 IEEE International Symposium on. IEEE, 2014.

[337] Almazyad, Abdulaziz S., et al. "A proposed scalable design and simulation of wireless sensor network-based long-distance water pipeline leakage monitoring system." Sensors 14.2 (2014): 3557-3577.

[338] Wykret, Thiago F., et al. "Evaluation and avoidance of interference in WSN: A multi-radio node prototype using Dynamic Spectrum Allocation.” Wireless Days (WD), 2013 IFIP. IEEE, 2013.

[339]

[340] Ziouva, Eustathia, and Theodore Antonakopoulos. "CSMA/CA performance under high traffic conditions: throughput and delay analysis." Computer communications 25.3 (2002): 313-321.

[341] Oh, Mi-Kyung, et al. "Cooperative synchronization and channel estimation in wireless sensor networks." Journal of Communications and Networks 7.3 (2005): 284-293.

[342] Wang, Tong, Rodrigo C. de Lamare, and Paul D. Mitchell. "Lowcomplexity set-membership channel estimation for cooperative wireless sensor networks." IEEE Transactions on Vehicular Technology 60.6 (2011): 2594-2607.

[343] Senel, Murat, et al. "A kalman filter based link quality estimation scheme for wireless sensor networks." Global Telecommunications Conference, 2007. GLOBECOM'07. IEEE. IEEE, 2007.

[344] Ribeiro, Alejandro, et al. "Kalman filtering in wireless sensor networks." IEEE Control Systems 30.2 (2010): 66-86.

[345] Sundararaman, Bharath, Ugo Buy, and Ajay D. Kshemkalyani. "Clock synchronization for wireless sensor networks: a survey." Ad hoc networks 3.3 (2005): 281-323.

[346] Wu, Yik-Chung, Qasim Chaudhari, and Erchin Serpedin. "Clock synchronization of wireless sensor networks." IEEE Signal Processing Magazine 28.1 (2011): 124-138.

[347] I. Hosni and N. Hamdi, "Cross layer optimization of end to end delay in WSN for smart grid communications," 2016 International Symposium on Signal, Image, Video and Communications (ISIVC), Tunis, 2016, pp. 217-223.doi: 10.1109/ISIVC.2016.7893990

[348] He, Jianping, et al. "Study of consensus-based time synchronization in wireless sensor networks." ISA transactions 53.2 (2014): 347-357.

[349] Elson, Jeremy, and Kay Rmer. "Wireless sensor networks: A new regime for time synchronization." ACM SIGCOMM Computer Communication Review 33.1 (2003): 149-154. 
[350] Bojic, Iva, and Kristian Nymoen. "Survey on synchronization mechanisms in machine-to-machine systems." Engineering Applications of Artificial Intelligence 45 (2015): 361-375.

[351] Simeone, Osvaldo, et al. "Distributed synchronization in wireless networks.” IEEE Signal Processing Magazine 25.5 (2008).

[352] M. Raza, H. Le-Minh, N. Aslam, S. Hussain and W. Ellahi, "A control channel based MAC protocol for time critical and emergency communications in Industrial Wireless Sensor Networks," 2017 International Conference on Communication, Computing and Digital Systems (C-CODE), Islamabad, 2017, pp. 122-126.doi: 10.1109/C-CODE.2017.7918914

[353] P. Radmand, A. Talevski, S. Petersen, and S. Carlsen, "Taxonomy of wireless sensor network cyber security attacks in the oil and gas industries, in Advanced Information Networking and Applications (AINA), 2010 24th IEEE Int. Conf., pp. 949957, 2010.

[354] J. P. Walters, Z. Liang, W. Shi, V. Chaudhary, "Wireless sensor network security: A survey, in Distributed, Grid, and Pervasive Computing, Yang Xiao Eds. Auerbach Publications, 2006.

[355] T. I. Jonas Olsson ¡ em $_{i} 6$ LoWPAN demystified ${ }_{i} / \mathrm{em}_{i} 2014$ [online] Available: http://www.ti.com/lit/wp/swry013/swry013.pdf.

[356] A. Perrig, J. Stankovic, and D. Wagner, Security in wireless sensor networks, ACM Commun., vol. 47, pp. 5357, June 2004.

[357] P. Radmand, A. Talevski, S. Petersen, and S. Carlsen, Taxonomy of wireless sensor network cyber security attacks in the oil and gas industries, in Advanced Information Networking and Applications (AINA), 2010 24th IEEE Int. Conf., pp. 949957, 2010.

[358] H. Li, Z. Jia and X. Xue, "Application and Analysis of ZigBee Security Services Specification," 2010 Second International Conference on Networks Security, Wireless Communications and Trusted Computing, Wuhan, Hubei, 2010, pp. 494-497. doi: 10.1109/NSWCTC.2010.261

[359] Hennebert, Christine, and Jessye Dos Santos. "Security protocols and privacy issues into 6LoWPAN stack: a synthesis." IEEE Internet of Things Journal 1.5 (2014): 384-398.

[360] Lu, Gang, Bhaskar Krishnamachari, and Cauligi S. Raghavendra. "An adaptive energy-efficient and low-latency MAC for data gathering in wireless sensor networks." Parallel and Distributed Processing Symposium, 2004. Proceedings. 18th International. IEEE, 2004.

[361] S. Liang, Y. Tang and Q. Zhu, "Passive Wake-up Scheme for Wireless Sensor Networks," Second International Conference on Innovative Computing, Informatio and Control (ICICIC 2007), Kumamoto, 2007, pp. 507-507. doi: 10.1109/ICICIC.2007.443

[362] Riggio, Roberto, Rasheed, Tinku M., Sicari, Sabrina, Performance Evaluation of an Hybrid Mesh and Sensor Network, IEEE International on Global Telecommunications, pp. 16, 2011.

[363] T. T. Vinh, T. N. Quynh, M. B. T. Quynh,Energy-Aware Mesh Routing Protocol For Wireless Sensor Networks, IEEE International Conference on Advanced Technologies for Communications, pp. 78 82, 2012.

[364] H.K.Le, D. Henriksson, T. Abdelzaher,A control theory approach to throughput optimization in multi-channel collection sensor networks, In IPSN, 2007.

[365] Y. Wu, S. Fahmy, N.B. Shroff,On the construction of a maximumlifetime data gathering tree in sensor networks: NP-completeness and approximation algorithm, In INFOCOM, 2008.

[366] Fan Yang, Chun He, Huai-zong Shao,Two Simplified Coding Schemes for Wireless Sensor Networks with Star Topologies, International Conference on Software, Telecommunications and Computer Networks (SoftCOM), pp. 185 189, 2010.

[367] Imad S. AlShawi, Lianshan Yan,Lifetime Enhancement in Wireless Sensor Networks Using Fuzzy Approach and A-Star Algorithm, IEEE Sensors Journal, Vol. 12, No. 10, pp. 3010 3018, 2012.

[368] Zhizhou Wu, Hao Chu, Yuqi Pan, Xiaoguang Yang, Bus Priority Control System Based on Wireless Sensor Network (WSN) and Zigbee, IEEE International Conference on Vehicular Electronics and Safety, pp. 148 151, 2006.

[369] A.S.Poornima, B.B.Amberker,Logical Ring based Key Management for Clustered Sensor Networks with Changing Cluster Head, International Conference on Signal Processing and Communications (SPCOM), pp. 1-5, 2010.

[370] Lambrou, T.P. Panayiotou, C.G.,A Survey on Routing Techniques Supporting Mobility in Sensor Networks, 5th International Conference on Mobile Ad-hoc and Sensor Networks (MSN), pp. 78 - 85, 2009.

[371] Sharma, Divya, Sandeep Verma, and Kanika Sharma. "Network topologies in wireless sensor networks: a review 1." (2013).

[372] Banimelhem, O., Khasawneh, S.,Grid-based Multi-path with Congestion Avoidance Routing (GMCAR) Protocol for Wireless Sensor Networks, IEEE International Conference on Telecommunication (ICT), pp. 131 136, 2009.
[373] Zurawski, R. Networked Embedded Systems; CRC Press: Boca Raton, FL, USA, 2009.

[374] Wireless Systems for Industrial Automation: Process Control and Related Applications, ISA100.11a Standard, 2009.

[375] Jagadeesan S., Parthasarathy V. (2012) Cross-Layer Design in Wireless Sensor Networks. In: Wyld D., Zizka J., Nagamalai D. (eds) Advances in Computer Science, Engineering \& Applications. Advances in Intelligent and Soft Computing, vol 166. Springer, Berlin, Heidelberg

[376] J. Wan, D. Yuan, and X. Xu, "A review of routing protocols in wireless sensor networks," in Wireless Communications, Networking and Mobile Computing, 2008. WiCOM'08. 4th International Conference on, 2008, pp. $1-4$.

[377] P. Kumar, M. Singh, and U. Triar, "A review of routing protocols in wireless sensor network," International Journal of Engineering Research \& Technology (IJERT), vol. 1, 2012.

[378] S. K. Singh, P. Kumar and J. P. Singh, "A Survey on Successors of LEACH Protocol,' in IEEE Access, vol. 5, no. , pp. 4298-4328, 2017. doi: 10.1109/ACCESS.2017.2666082

[379] J. Hao, B. Zhang and H. T. Mouftah, "Routing protocols for duty cycled wireless sensor networks: A survey," in IEEE Communications Magazine, vol. 50, no. 12, pp. 116-123, December 2012. doi: 10.1109/MCOM.2012.6384460

[380] M. Asif, S. Khan, R. Ahmad, M. Sohail and D. Singh, "Quality of Service of Routing Protocols in Wireless Sensor Networks: A Review," in IEEE Access, vol. 5, pp. 1846-1871, 2017. doi: 10.1109/ACCESS.2017.2654356

[381] X. Liu, "Atypical Hierarchical Routing Protocols for Wireless Sensor Networks: A Review," in IEEE Sensors Journal, vol. 15, no. 10, pp. 53725383, Oct. 2015. doi: 10.1109/JSEN.2015.2445796

[382] N. A. Pantazis, S. A. Nikolidakis and D. D. Vergados, "Energy-Efficient Routing Protocols in Wireless Sensor Networks: A Survey," in IEEE Communications Surveys \& Tutorials, vol. 15, no. 2, pp. 551-591, Second Quarter 2013.

[383] S. Murphy, L. Aceves, An Efficient Routing Protocol for Wireless Networks, Mobile Networks and Applications, ACM Journal, USA Hingham, 1996, Vol. 1, Issue 2, pp. 183-197.

[384] B. Bellur, R. Ogier, A Reliable, Efficient Topology Broadcast Protocol for Dynamic Networks, In Proc. IEEE INFOCOM 99, New York, USA, 1999, vol.1, pp.178-186.

[385] S. Bali, Performance Comparisons of DSR and TORA, Wireless Networks and Mobile Computing, South Carolina, 2001, pp. 10-15

[386] H. Lim, C. Kim, Flooding in Wireless Ad Hoc Networks, Computer Communications, 2001, Vol. 24, Issue 3, pp. 353-363.

[387] S. Hedetniemi, A. Liestman, A Survey of Gossiping and Broadcasting in Communication Networks, Book:Networks, 1998, Vol. 18, Issue 4, pp. 319-349.

[388] S. Giannoulis, C. Antonopoulos, E. Topalis, S. Koubias, ZRP versus DSR and TORA: A comprehensive survey on ZRP performance, In Proc. 10th IEEE Conference on Emerging Technologies and Factory Automation, Catania, 2005, pp. 1017-1024.

[389] D. A. Vidhate, A. K. Patil, S. S. Pophale, Performance Evaluation of Low Energy Adaptive Clustering Hierarchy Protocol for Wireless Sensor Networks, In Proc. International Conference and Workshop on Emerging Trends in Technology (ICWET 2010)TCET, Mumbai, India, 2010, pp. 59-63.

[390] A. Manjeshwar, D. Agrawal, Teen: A Routing Protocol for Enhanced Efficiency in Wireless Sensor Networks, In Proc. 15th International Parallel and Distributed Processing Symposium (IPDPS01) Workshops, USA, California, 2001, pp. 2009-2015.

[391] S. Lindsey, C.Raghavendra, PEGASIS: Power-Efficient GAthering in Sensor Information Systems, In Proc. IEEE Aerospace Conference, USA, Montana, 2002, Vol. 3, pp. 1125-1130.

[392] A. Manjeshwar, D. Agrawal, APTEEN: A Hybrid Protocol for Efficient Routing and Comprehensive Information Retrieval in Wireless Sensor Networks, In Proc. International Parallel and Distributed Processing Symposium, Florida, 2002, pp. 195-202.

[393] Y. Yuan, Z. He, M. Chen, Virtual MIMO-based Cross-layer Design for Wireless Sensor Networks, IEEE Trans. Veh. Technol., 2006, Vol. 55, Issue 3, pp. 856-864.

[394] Q. Li, J. Aslam, D. Rus, Hierarchical Power-aware Routing in Sensor Networks, In Proc. DIMACS Workshop on Pervasive Networking, California, 2001, pp. 25-27.

[395] H. Cheng, G. Yang, S. Hu, NHRPA: A Novel Hierarchical Routing Protocol Algorithm for Wireless Sensor Networks, China Universities of Posts and Telecommunications, 2008, Vol. 15, Issue 3, pp. 75-81. 
[396] C. H. Lung, C. Zhou, Using Hierarchical Agglomerative Clustering in Wireless Sensor Networks: An Energy-efficient and Flexible Approach, Ad Hoc Networks, 2010, Vol. 8, Issue 3, pp. 328-344.

[397] H. Luo, F. Ye, J. Cheng, S. Lu, L. Zhang, TTDD: Two-Tier DataDissemination in Large-Scale Wireless Sensor Networks, Wireless Networks, Springer Netherlands, 2005, Vol. 11, Issue 1, pp. 161-175.

[398] Y. Yao, J. Gehrke, The Cougar Approach to In-Network Query Processing in Sensor Networks, SIGMOD Record, 2002, Vol. 31, Issue 3, pp. $9-18$.

[399] J. Newsome, D. Song, GEM: Graph EMbedding for Routing and Datacentric Storage in Sensor Networks Without Geographic Information, In Proc. 1st International Conference on Embedded Networked Sensor Systems, California, USA, 2003, pp. 76-88.

[400] Y. Yu, R. Govindan, D. Estrin, Geographical and Energy Aware Routing: A Recursive Data Dissemination Protocol for Wireless Sensor Networks, UCLA Computer Science Department Technical Report, 2001, pp. 1-11

[401] B. Blum, T. He, S. Son, J. Stankovic, IGF: A State-Free Robust Communication Protocol for Wireless Sensor Networks, Technical Report CS-2003-11, Department of Computer Science, University of Virginia, USA, 2003.

[402] G. Lukachan, M. Labrador, SELAR: Scalable Energy-Efficient Location Aided Routing Protocol for Wireless Sensor Networks, In Proc. 29th Annual IEEE International Conference on Local Computer Networks, USA, Florida, 2004, pp. 694-695.

[403] D. Chen, P. Varshney, On-demand Geographic Forwarding for Data Delivery in Wireless Sensor Networks, Computer Communications, 2007, Vol. 30, Issue 14-15, pp. 29542967.

[404] N. M. Khan, Z. Khalid, and G. Ahmed, "Gradient cost establishment (grace) for an energy-aware routing in wireless sensor networks," EURASIP Journal on Wireless Communications and Networking, vol. 2009, pp. 1-15, 2009.

[405] C. Schurgers and M. B. Srivastava, "Energy efficient routing in wireless sensor networks," in Military communications conference, 2001. MILCOM 2001. Communications for network-centric operations: Creating the information force. IEEE, 2001, pp. 357-361.

[406] P. Huang, H. Chen, G. Xing, and Y. Tan, "SGF: a state-free gradientbased forwarding protocol for wireless sensor networks," ACM Transactions on Sensor Networks (TOSN), vol. 5, p. 14, 2009.

[407] J. Guo, P. Orlik, J. Zhang, and K. Ishibashi, "Reliable routing in large scale wireless sensor networks," in Ubiquitous and Future Networks (ICUFN), 2014 Sixth International Conf on, 2014, pp. 99-104.

[408] [23]. G. Yi, S. Guiling, L. Weixiang and P. Yong Recluster-LEACH: A recluster control algorithm based on density for wireless sensor network 2nd International Conference on Power Electronics and Intelligent Transportation System vol.3, pp.198-202, 2009.

[409] [38]. L. Subramanian and R. H. Katz, An Architecture for Building Self Configurable Systems, Proc. IEEE/ACM Wksp. Mobile Ad Hoc Net. and Comp., Boston, MA, Aug. 2000

[410] M. Chu, H. Haussecker, and F. Zhao: Scalable Information-Driven Sensor Querying and Routing for ad hoc Heterogeneous Sensor Networks, The International Journal of High Performance Computing Applications, Vol. 16, No. 3 ( August 2002)

[411] S. Lindsey, C. S. Raghavendra, and K. M. Sivalingam, "Data gathering algorithms in sensor networks using energy metrics", IEEE ransactions on Parallel and Distributed Systems, vol. 13, no. 9, Sept. 2002, pp. 924-935.

[412] M. Chen, S. Gonzalez, Y. Zhang, V. Leung, Multi-agent Itinerary Planning in Wireless Sensor Networks, Computer Science, 2009, Vol. 22, Issue 10, pp. 584-597.

[413] M. Chen, L. Yang, T. Kwon, L. Zhou, M. Jo, Itinerary Planning for Energy-efficient Agent Communication in Wireless Sensor Networks, IEEE Trans. Veh. Technol., 2011, Vol. 60, Issue 7, pp. 1-8.

[414] J. Luo, and J.- P. Hubaux, "Joint mobility and routing for lifetime elongation in wireless sensor networks", Proceedings IEEE INFOCOM'05, vol. 3, Miami, FL, Mar. 2005, pp. 1735-1746.

[415] R.C. Shah, S. Roy, S. Jain, and W. Brunette, "Data MULEs: Modeling a three-tier architecture for sparse sensor networks ", Proceedings SN P A '03, Anchorage, AK, May 2003, pp. 30-41.

[416] Kim, Hyung Seok, Tarek F. Abdelzaher, and Wook Hyun Kwon. "Minimum-energy asynchronous dissemination to mobile sinks in wireless sensor networks." Proceedings of the 1st international conference on Embedded networked sensor systems. ACM, 2003.

[417] W. Chang, G. Cao, and T. La Porta, "Dynamic proxy tree-based data dissemination schemes for wireless sensor networks", Proceedings IEEE MASS'04, Fort Lauderdale, FL, Oct. 2004, pp. 21-30.
[418] F. Ye, G. Zhong, S. Lu, L. Zhang, GRAdient Broadcast: A Robust Data Delivery Protocol for Large Scale Sensor Networks, Wireless Networks (WINET), 2005, Vol. 11, Issue 3, pp. 285-298.

[419] Y. Wang, C. Tsai, H. Mao, HMRP: Hierarchy-Based Multipath Routing Protocol for Wireless Sensor Networks, Science and Engineering, 2006 , Vol. 9, Issue 3, pp. 255-264.

[420] F. Ye et al., A Two-Tier Data Dissemination Model for Large-Scale Wireless Sensor Networks, Proc. ACM/IEEE MOBICOM, 2002.

[421] Kiri, Yuichi, Masashi Sugano, and Masayuki Murata. "Self-organized data-gathering scheme for multi-sink sensor networks inspired by swar$\mathrm{m}$ intelligence." Self-Adaptive and Self-Organizing Systems, 2007. SASO'07. First International Conference on. IEEE, 2007.

[422] Kulik, Joanna, Wendi Heinzelman, and Hari Balakrishnan. "Negotiation-based protocols for disseminating information in wireless sensor networks." Wireless networks 8.2/3 (2002): 169-185.

[423] Guo, Wenjing, Wei Zhang, and Gang Lu. "A comprehensive routing protocol in wireless sensor network based on ant colony algorithm." Networks Security Wireless Communications and Trusted Computing (NSWCTC), 2010 Second International Conference on. Vol. 1. IEEE, 2010.

[424] Chu M, Haussecker H, Zhao F. Scalable information driven sensor querying and routing for ad hoc heterogeneous sensor networks, The International Journal of High Performance Computing Applications, 2002;16(3):293313

[425] Shah, Rahul C., and Jan M. Rabaey. "Energy aware routing for low energy ad hoc sensor networks." Wireless Communications and Networking Conference, 2002. WCNC2002. 2002 IEEE. Vol. 1. IEEE, 2002.

[426] Braginsky, David, and Deborah Estrin. "Rumor routing algorthim for sensor networks." Proceedings of the 1st ACM international workshop on Wireless sensor networks and applications. ACM, 2002.

[427] S. Lee, Y. Yu, S. Nelakuditi, Z. Zhang, C. Chuah, Proactive vs Reactive Approaches to Failure Resilient Routing, In Proc. 23rd nnualJoint Conference of the IEEE Computer and Communications Societies, China, 2004, Vol. 1, pp. 176-186.

[428] He, Tian, et al. "SPEED: A stateless protocol for real-time communication in sensor networks." Distributed Computing Systems, 2003. Proceedings. 23rd International Conference on. IEEE, 2003.

[429] Paone, Maurizio, et al. "A multi-sink swarm-based routing protocol for wireless sensor networks." Computers and Communications, 2009. ISCC 2009. IEEE Symposium on. IEEE, 2009.

[430] Chen, Wei-Ming, et al. "Jumping ant routing algorithm for sensor networks." Computer Communications 30.14 (2007): 2892-2903

[431] A. Manjeshwar and D. P. Agarwal, APTEEN: A Hybrid Protocol for Efficient Routing and Comprehensive Information Retrieval in Wireless Sensor Networks, Proc. Intl. Parallel and Distrib. Proc. Symp., pp. 195202.

[432] Intanagonwiwat, Chalermek, et al. "Directed diffusion for wireless sensor networking." IEEE/ACM Transactions on Networking (ToN) 11.1 (2003): 2-16

[433] N. Sadagopan, B. Krishnamachari, A. Helmy, Active Query Forwarding in Sensor Networks (ACQUIRE), Ad Hoc Networks, 2005, Vol. 3, Issue 1, pp. 91-113.

[434] C. M. Cordeiro, D. P. Agrawal, Book: Ad hoc and Sensor Networks: Theory and Applications Edition, World Scientific, 2006.

[435] J. Ben-Othman and B. Yahya, "Energy efficient and QoS based routing protocol for wireless sensor networks," J. Parallel Distrib. Comput., vol. 70, no. 8, pp. 849-857, 2010.

[436] S. Basagni, I. Chlamtac, V. R. Syrotiuk and B. A Woodward. A distance routing effect algorithm for mobility (DREAM), In ACM/IEEE International Conference on Mobile Computing and Networks (MOBICOM98), pages 76-84, October 1999, Dallas, Texas.

[437] Y.B. Ko and N.H. Vaidya, Location-aided routing (LAR) in mobile ad hoc networks, ACM/IEEE International Conference on Mobile Computing and Networking (MobiCom), 1998, pp. 66-75.

[438] J. N. Al-Karaki et al., Data Aggregation in Wireless Sensor Network$\mathrm{s}$ Exact and Approximate Algorithms, Proc. IEEE Wksp. High Perf. Switching and Routing 2004, Phoenix, AZ, Apr. 1821, 2004

[439] K. Sohrabi and J. Pottie, Protocols for Self-Organization of a Wireless Sensor Network, IEEE Pers. Commun., vol. 7, no. 5, 2000, pp. 1627.

[440] K. Sohrabi and J. Pottie, Protocols for Self-Organization of a Wireless Sensor Network, IEEE Pers. Commun., vol. 7, no. 5, 2000, pp. 1627.

[441] D. Cevizovic, S. Galovic, S. Zekovic, Z. Ivic, Boundary Between Coherent and Noncoherent Small Polaron Motion: Influence of the Phonon Hardening, Physica B:Condensed Matter, 2009, Vol. 404, Issue 2, pp. 270-274. 
[442] Kang, Jaewon, Yanyong Zhang, and Badri Nath. "TARA: topologyaware resource adaptation to alleviate congestion in sensor networks." IEEE Transactions on Parallel and Distributed Systems 18.7 (2007).

[443] Dinesh Thakur, Open Loop Congestion Control (2017), Available:[http://ecomputernotes.com/computernetworkingnotes/communicati on-networks/what-is-congestion-control-describe-the-congestion-controlalgorithm-commonly-used]

[444] Ghaffari, Ali. "Congestion control mechanisms in wireless sensor networks: A survey." Journal of network and computer applications 52 (2015): 101-115.

[445] Chakravarthi R, Gomathy C,Sebastian SK,Pushparaj K,Mon VB. A survey on congestion control in wirelesssensor networks .Int $\mathrm{J}$ Comput Sci Commun 2010;1(1):1614.

[446] Doherty, Lance Robert, Jonathan Simon, and Zhenqiang Ye. "Congestion control for wireless sensor networks." U.S. Patent No. 8,189,479. 29 May 2012.

[447] Wang, Chonggang, et al. "Upstream congestion control in wireless sensor networks through cross-layer optimization." IEEE Journal on selected areas in Communications 25.4 (2007).

[448] Polibol: Process capabilities (2017) Available: [http://www.polibol.es/eng/procesos.htm]

[449] Smart Factory: Reducing Maintenance Costs and Ensuring Quality in the Manufacturing Process, (2017) Available: [http://www.libelium.com/smart-factory-reducing-maintenance-costsensuring-quality-manufacturing-process/]

[450] Libelium Sensors Connect with Microsoft Azure Cloud Platform, (2017) Available: [http://www.libelium.com/libelium-sensors-connectmicrosoft-azure-cloud-platform/]

[451] Meshlium Xtreme. [http://www.libelium.com/products/meshlium/

(2017) Available:

452] Honeywell Wireless Solution Improves Steel Mill Performance (2017) Available: [https://www.honeywellprocess.com/library/.../case.../successstory-nucor-wireless.pdf]

[453] Beth Wozniak, Honeywell Wireless Sensor Networks, 2009. Available: [https://www.oecd.org/sti/ieconomy/43017542.pdf]

[454] OneWireless Network - Multiprotocol Wireless IO \& System, (2017), Available: [https://www.honeywellprocess.com/enUS/explore/products/wireless/OneWireless-Network/pages/default.aspx]

[455] Ross Yu, Reliable Wireless Sensor Network Streamlines Manufacturing Operations (Jan 2017) Available: [http$\mathrm{s}: / / \mathrm{www}$. youtube.com/watch? $\mathrm{v}=\mathrm{kM} 3 \mathrm{z} 4 \mathrm{w} 5 \mathrm{NoW} 8]$

[456] Wireless Sensor Network Streamlines Semiconductor Manufacturing Operations (2017) Available: [www.linear.com/docs/47541]

[457] Issariyakul, Teerawat, and Ekram Hossain. Introduction to network simulator NS2. Springer Science \& Business Media, 2011.

[458] Baldo, Nicola, et al. "ns2-MIRACLE: a modular framework for multitechnology and cross-layer support in network simulator 2." Proceedings of the 2nd international conference on Performance evaluation methodologies and tools. ICST (Institute for Computer Sciences, Social-Informatics and Telecommunications Engineering), 2007.

[459] Jianliang Z. and M. J. Lee. A comprehensive performance study of IEEE 802.15.4, Sensor Network Operations, IEEE Press, pp: 1-14, 2004

[460] Comparison between NS2 and NS3 (2017). Available: [https://www.researchgate.netfile.PostFileLoader.html?id=5372cf86d11b8 b3b5d8b45ae\&assetKey=AS\%3A273533144371217\%401442226791690]

[461] OMNeT++ Discrete Event Simulator, (2017) Available: [https://omnetpp.org]

[462] Dai, Xuewu, et al. "Development and validation of a simulator for wireless data acquisition in gas turbine engine testing." IET Wireless Sensor Systems3.3 (2013): 183-192.

[463] A. Al-Yami, W. Abu-Al-Saud and F. Shahzad, "On Industrial Wireless Sensor Network (IWSN) and Its Simulation Using Castalia," 2016 UKSim-AMSS 18th International Conference on Computer Modelling and Simulation (UKSim), Cambridge, 2016, pp. 293-298. doi: 10.1109/UKSim.2016.51

[464] N. G. Palan and A. P. Khadilkar, "Media Access Control protocol modelling for Mobile Sensor Network-using OMNeT++ -MiXiM network simulator," International Conference on Sustainable Energy and Intelligent Systems (SEISCON 2011), Chennai, 2011, pp. 641-644.

[465] End-to-end perspective of application and network performance with OPNET technologies, (2017) Available: [https://www.riverbed.com/gb/products/steelcentral/opnet.html]

[466] C. Marghescu, M. Pantazica, A. Brodeala and P. Svasta, "Simulation of a wireless sensor network using OPNET," 2011 IEEE 17th International Symposium for Design and Technology in Electronic Packaging (SIITME), Timisoara, 2011, pp. 249-252. doi: 10.1109/SIITME.2011.6102728
[467] QualNet Network Simulator Software (2017) Available: [http://web.scalable-networks.com/qualnet-network-simulator-software]

[468] Simulation of wireless ad-hoc sensor networks with QualNet, (2017) Available: [https://www-user.tu-chemnitz.de/ doto/pdf/wirelesssimulation-with-qualnet.pdf]

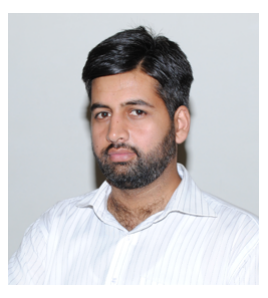

Mohsin Raza received his B.S and M.S. degrees in Electronic Engineering from Mohammad Ali Jinnah University, Islamabad, Pakistan. Currently he is a $\mathrm{PhD}$ student in Department of Mathematics, Physics and Electrical Engineering, Northumbria University, Newcastle, UK. Prior to this he worked as a Lecturer in department of Electronic Engineering at Mohammad Ali Jinnah University, Islamabad from 2010 to 2015 and Hardware Support Engineer at USS in 2009

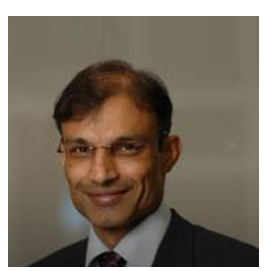

Dr. Nauman Aslam is a Reader in the Department of Computer Science and Digital Technologies. He joined Northumbria University in August 2011. Dr. Nauman received his PhD in Engineering Mathematics from Dalhousie University, Halifax, Nova Scotia, Canada in 2008. He was awarded Master of Engineering Degree in Internetworking from Dalhousie University in 2003; and BSc in Mechanical Engineering from University of Engineering and Technology, Lahore, Pakistan in 1993. Prior to joining Northumbria University he worked as an Assistant Professor at Dalhousie University, Canada from 2008 - 2011. Currently, he also holds an adjunct assistant professor position at Dalhousie University.

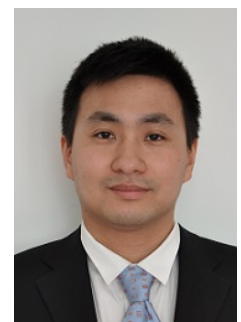

Dr. Hoa Le-Minh received his BEng in Telecommunications in 1999 at Bach Khoa University of Hochiminh city, Vietnam, and then worked as lecturer in Telecommunications Department at the same University. He received MSc in 2003 and $\mathrm{PhD} 2007$ degrees in Telecommunications in Munich University of Technology (TUM), Germany and Northumbria University, Newcastle, UK, respectively. Hoa worked as a research assistant in Siemens AG, Munich, Germany during 2002 - 2004 and postdoctoral research fellow in University of Oxford, UK, from 2007 to 2010. Since 2010 he became the senior lecturer at Northumbria University. His research areas are wireless communications, optical wireless communications, sensor network and Smartphone technology. He has published over 150 papers in these fields. Currently he is the Chairman of IEEE ComSoc UK and Ireland. 


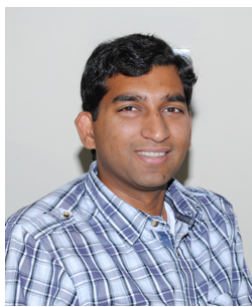

Dr. Sajjad Hussain received his MS degree from SUPELEC, Gif-sur-Yvette, France and PhD degree from University of Rennes 1, France in Wireless Communication and Signal Processing. He is currently Lecturer at School of Engineering, University of Glasgow, UK. Prior to this he was Associate Professor at Capital University of Science and Technology. He also worked as Assistant Professor at National university of Science and Technology, Rawalpindi, Pakistan. His main research interests include spectrum sensing, security, and cross layer optimization in cognitive radios and wireless networks.

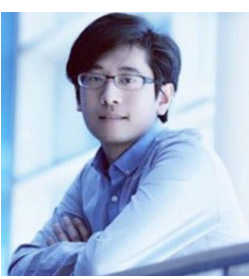

Dr. Yue Cao received the $\mathrm{PhD}$ degree from the Institute for Communication Systems (ICS), at University of Surrey, Guildford, UK in 2013. He was a Research Fellow at the ICS until September 2016, and Lecturer in Department of Computer and Information Sciences, at Northumbria University, Newcastle upon Tyne, UK until July 2017, and currently the Senior Lecturer since August 2017. His research interests focus on DTNs, E-Mobility, QoS/QoE in 5G.

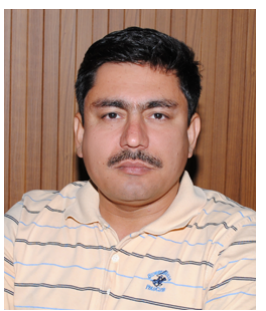

Dr. Noor M. Khan has vast research experience in the field of Wireless Communications Engineering. His main research areas are Cellular and Mobile Communication Systems, Channel Modeling, Fading Channel Characterization, Smart Antennas, MIMO systems, Adaptive Signal Processing and Wireless Sensor Networks. He received his $\mathrm{PhD}$ in Electrial Engineering from University of New South Wales (UNSW), Sydney, NSW, Australia. He is currently a Professor in the Department of Electrical Engineering at Capital University of Science and Technology Islamabad, Pakistan. Prior to joining Capital University of Science and Technology he has worked as an Assistant Professor at Ghulam Ishaq Khan Institute of Engineering Sciences and Technology, Topi, Khyber Pakhtunkhwa, Pakistan from 2006-2007. He has also worked with Pakistan Telecommunications Company Ltd. (PTCL), Pakistan 2001-2006 and World Call Communications (Pvt.) Ltd., Lahore, Pakistan 1999-2000. 